\title{
SECOND-ORDER FUNCTIONAL-DIFFERENCE EQUATIONS. II: SCATTERING FROM A RIGHT-ANGLED CONDUCTIVE WEDGE FOR $E$-POLARIZATION
}

\author{
by Y. A. ANTIPOV ${ }^{\dagger}$
}

(Department of Mathematics, Louisiana State University, Baton Rouge, LA 70803, USA)

and V. V. SILVESTROV

(Department of Mathematics, Chuvash State University, Cheboksary 428015, Russia)

[Received 1 September 2003. Revise 11 February 2004]

\begin{abstract}
Summary
In part I, a new method for solving functional-difference equations of the second order was proposed. The shift of the equation was assumed to coincide with the period of the coefficients. The method is based on the theory of the Riemann-Hilbert problem on a hyperelliptic surface and the Jacobi inversion problem. The procedure is applicable to any finite number of zeros of the discriminant of the equation in the strip. It yields the general single-valued meromorphic solution.

In the present paper, electromagnetic scattering by a right-angled magnetically conductive wedge is analysed. The physical problem reduces to a second-order difference equation with $2 \pi$-periodic coefficients and with the shift $\pi$. A rigorous procedure for constructing the general solution is proposed. It consists of two steps. First, an auxiliary equation with the shift $2 \pi$ and the period $\pi$ is derived and solved by the method proposed in part I (the corresponding Riemann surface is a torus). Next, necessary and sufficient conditions for the solution of the auxiliary equation to satisfy the governing equation are derived. These conditions separate the general solution of the main equation from those solutions of the auxiliary equation which fail to satisfy the governing difference equation. In addition, the particular case of no branch points is analysed by the machinery of the Riemann-Hilbert problem for a segment on the complex plane. A high-frequency asymptotic expression for the electric field is presented. Numerical results for the backscattering coefficient are reported.
\end{abstract}

\section{Introduction}

In recent years considerable attention has been focused on electromagnetic scattering by wedges whose sheets have a finite and non-zero electric resistivity. Such sheets support only an electric current with the strength proportional to the value of the tangential electric field at the surface. The electromagnetic dual of an electrically resistive sheet is (Senior and Volakis (1)) a magnetically conductive one which simulates a thin layer of lossy material. On the boundary, in the case of an electrically resistive sheet, the electric field is continuous, the normal derivative of the field

\footnotetext{
† $\langle$ antipov@math.lsu.edu $\rangle$

* $\langle$ sil@chuvsu.ru $\rangle$
}

Q. JI Mech. Appl. Math. (2004) 57 (2), 267-313 @) Oxford University Press 2004; all rights reserved. 
is discontinuous and its jump is proportional to the electric field. If the sheet is magnetically conductive and its conductivity is finite and non-zero, then the electric field is discontinuous. Its normal derivative is continuous and it is proportional to the jump of the field.

By using the Maliuzhinets method (2) based on the use of the Sommerfeld integral representation of the electric field, the corresponding boundary conditions for the above problems may be brought into second-order functional-difference equations with periodic coefficients. In general, the shift in the equation and the period of the coefficients are not the same. Even in the simplest case of the wedge, namely for a right-angled wedge, the period is twice as much as the shift. For this geometry, when one of the sheets is electrically resistive and the second one is perfectly electrically conductive, Demetrescu et al. (3) have derived the governing difference equation of the second order with $2 \pi$ periodic coefficients and the shift $\pi$ whose solution has to be an odd function. A similar equation has been derived (4) for the problem of diffraction by a two-sided impedance plane with a resistive sheet attached to it. The authors of these papers have reduced the governing equations to solution of auxiliary equations of the second order with $\pi$-periodic coefficients and the shift $2 \pi$. Each solution of the initial equation satisfies the auxiliary equation but the inverse statement, in general, is not correct. Therefore, to solve the physical problem, one needs

(i) a single-valued meromorphic solution of the auxiliary equation, and

(ii) a mechanism for separating odd single-valued meromorphic solutions of the governing equation from mock solutions which satisfy the auxiliary equation only.

In $(\mathbf{3}, \mathbf{4})$, the authors found a non-physical multi-valued solution (for comments see $(\mathbf{5}, \mathbf{6})$ ). Also they have proposed to represent the general odd solution $f(s)$ of the main equation in the form $f(s)=f_{*}(s)-f_{*}(-s)$, where $f_{*}(s)$ is defined through a partial solution of the auxiliary equation and a periodic meromorphic function with prescribed properties. However, as it is shown in the Appendix below, this method does not produce the general solution of the main equation.

Senior and Legault $(\mathbf{5}, \mathbf{7})$ have proposed another method that can be applied for constructing a partial singled-valued meromorphic solution of the equation whose shift is a multiple of the period of the coefficients. At the first stage, this method reduces the second-order difference equation to a pair of first-order difference equations. Then by logarithmic differentiation of these equations and elimination of the polar and cyclic periods of the solution of the first-order equations, the authors construct a solution that is single-valued on a Riemann surface. These derivations ultimately lead to a branch-free partial solution of the second-order equation, the auxiliary equation for the governing one. We notice that the method $(\mathbf{5 , 7})$ is worked out either for genus $\rho=1$, or for genus three, when the surface has a special symmetry, and the problem is solvable in terms of elliptic functions (reducible to the case $\rho=1$ ). The general solution of the auxiliary equation with prescribed poles,

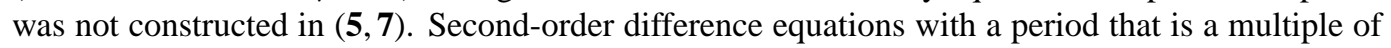
the shift, have not been analysed in those papers either.

In the present paper we aim to

- derive a governing equation of the electromagnetic problem of diffraction by a right-angled wedge when one of the sheets is magnetically conductive and the second one is perfectly electrically conductive;

- construct the general single-valued meromorphic solution of the corresponding auxiliary equation with shift equal to $2 \pi$ and $\pi$-periodic coefficients. For these purposes, we use a new method based on the machinery of the Riemann-Hilbert problem on a hyperelliptic surface proposed in the first part of this investigation (6); 
- work out a rigorous method for the general solution of the governing second-order equation with $2 \pi$-periodic coefficients when the shift is equal to $\pi$;

- find a closed-form solution of the problem on $E$-polarization of a right-angled magnetically conductive wedge.

The article is organized as follows. In section 2, the physical problem is formulated. Section 3 brings in two functional-difference equations of the second order. The first one, the governing equation, has shift $\pi$ and $2 \pi$-periodic coefficients. The second one is an auxiliary equation with shift $2 \pi$ and $\pi$-periodic coefficients. The general single-valued meromorphic solution of the auxiliary equation is found in section 4. The derivations are based on the results of the first part of this study (6). Then, section 5 offers a technique for the general solution of the governing equation that is required to be odd. The main idea of the method is to use the general solution of the auxiliary equation on the axis of symmetry of the strip and substitute it into the main equation. Then deduce the necessary and sufficient conditions for arbitrary $2 \pi$-meromorphic functions involved in the general solution of the auxiliary equation in order that the main equation is satisfied. Analytical continuation of these conditions from the contour into the whole complex plane completes the procedure. In section 6, we show that the spectral functions found belong to the prescribed class of solutions and we find arbitrary constants from additional physical conditions. A detailed analysis of the particular case when there are no branch points is presented in section 7. To find the general solution of the auxiliary second-order equation, we solve two scalar Riemann-Hilbert problems on the segment $[-1,1]$. So, the solution of the Riemann-Hilbert problem on a Riemann surface is bypassed. This method, proposed in section 7.1, can be regarded as an alternative to the classical expansion in terms of the Maliuzhinets functions for difference equations when the discriminant of the equation does not have zeros of odd order. In section 8 we derive high-frequency asymptotics of the electric field. We construct the reflected, transmitted, surface and diffracted waves. Numerical results are presented for the backscattering coefficient. In Appendix A, for the simplest case of no branch points, we show that the use of the method (3) produces less constants than are required by the physical problem. Appendix B presents formulae used for numerical calculations of the backscattering coefficient.

\section{Formulation}

The problem to be considered is the two-dimensional one presented in Fig.1. The half-plane $\{0<$ $r<\infty, \varphi=3 \pi / 4 \pm 0\}$ is a magnetically conductive sheet with the conductivity $R_{m}$. The second sheet $\{0<r<\infty, \varphi= \pm \pi+\pi / 4 \mp 0\}$ is electrically perfectly conducting (opaque) with the resistivity $R_{e}=0$. The system is illuminated by the $E$-polarized plane wave

$$
E_{z}^{i}=e^{i k_{0} r \cos \left(\varphi-\varphi_{0}\right)},
$$

where $(r, \varphi)$ is a point of observation, $k_{0}$ is the wave number, $\varphi_{0}$ is the incident angle. On the second sheet, the electric field $E_{z}$ is continuous and it vanishes:

$$
\left.E_{z}\right|_{\varphi=5 \pi / 4-0}=\left.E_{z}\right|_{\varphi=-3 \pi / 4+0}=0, \quad 0<r<\infty .
$$

As for the first sheet, the electric field $E_{z}$ is discontinuous on it, and its jump is proportional to the magnetic field $H_{\rho}$ (Senior and Volakis (1))

$$
\left.H_{\rho}\right|_{\varphi=3 \pi / 4 \pm 0}=-R_{m}\left[\left.E_{z}\right|_{\varphi=3 \pi / 4-0}-\left.E_{z}\right|_{\varphi=3 \pi / 4+0}\right] .
$$




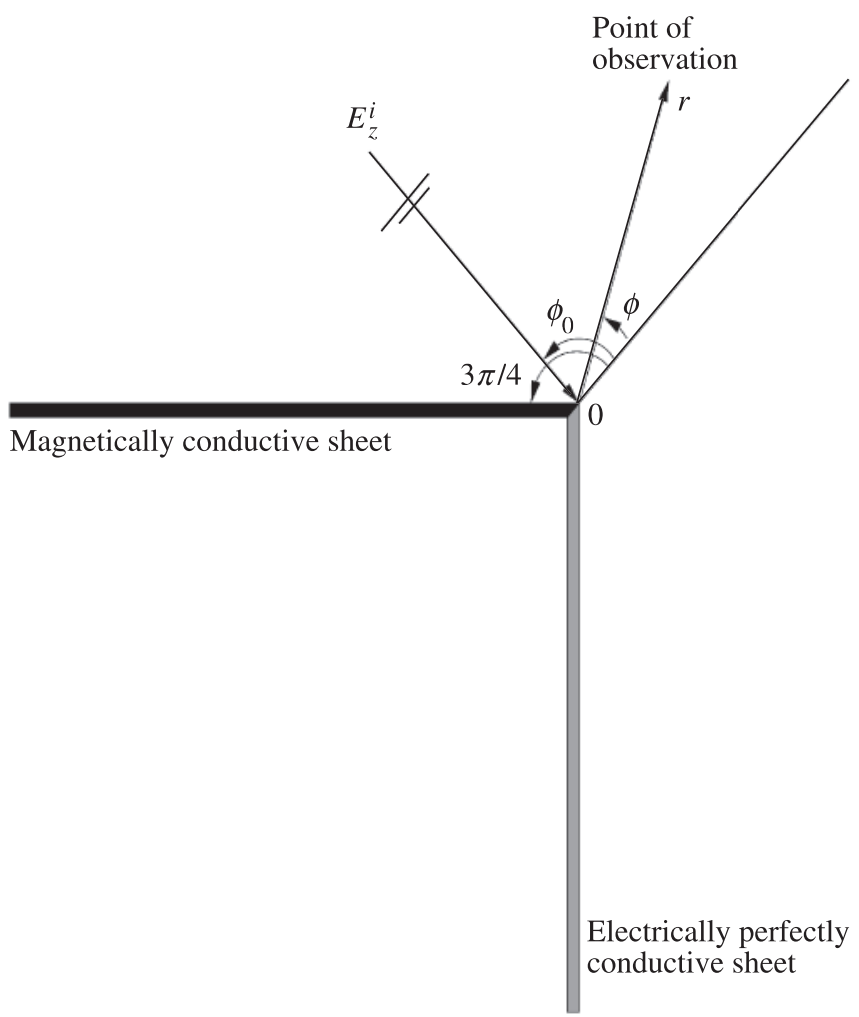

Fig. $1 E$-polarization of a conductive wedge

By expressing the component $H_{\rho}$ through the normal derivative of the electric field

$$
Z_{0} H_{\rho}=-\frac{1}{i k_{0} r} \frac{\partial E_{z}}{\partial \varphi}
$$

we derive the boundary conditions

$$
\begin{gathered}
\left.\frac{2}{r} \frac{\partial}{\partial \varphi} E_{z}\right|_{\varphi=3 \pi / 4-0}-i k_{0} \gamma\left(\left.E_{z}\right|_{\varphi=3 \pi / 4-0}-\left.E_{z}\right|_{\varphi=3 \pi / 4+0}\right)=0, \quad 0<r<\infty, \\
\left.\frac{\partial E_{z}}{\partial \varphi}\right|_{\varphi=3 \pi / 4-0}=\left.\frac{\partial E_{z}}{\partial \varphi}\right|_{\varphi=3 \pi / 4+0}, \quad 0<r<\infty,
\end{gathered}
$$

where $\gamma=2 R_{m} Z_{0} \neq 0, Z_{0}$ is the intrinsic impedance of the medium. Generally, the parameter $\gamma=\sin \theta$ is complex and $0<\operatorname{Re} \theta<\pi / 2$. 
Everywhere in the medium apart from the two sheets, the electric field $E_{z}$ satisfies the Helmholtz equation

$$
\left(\nabla^{2}+k_{0}^{2}\right) E_{z}=0
$$

To satisfy the differential equation (2.6) we represent the total field in the form of the Sommerfeld integrals (Maliuzhinets (2))

$$
\begin{gathered}
E_{z}(r, \varphi)=\frac{1}{2 \pi i} \int_{\Gamma} e^{i k_{0} r \cos s} \mathcal{S}_{1}(s+\varphi) d s, \\
E_{z}(r, \varphi)=\frac{1}{2 \pi i} \int_{\Gamma} e^{i k_{0} r \cos s} \mathcal{S}_{2}(s+\varphi-\pi) d s, \quad \frac{3 \pi}{4}<\varphi<\frac{5 \pi}{4},
\end{gathered}
$$

where $\Gamma$ is the Sommerfeld double loop contour symmetric with respect to the origin. The asymptotes for its branches are $s=3 \pi / 2$ and $s=-\pi / 2$ for the upper loop and $s=\pi / 2$ and $s=-3 \pi / 2$ for the lower one. The function $\mathcal{S}_{1}(s)$ is analytic everywhere in the strip $|\operatorname{Re} s|<3 \pi / 4$ and continuous in the strip up to the boundary $|\operatorname{Re} s|=3 \pi / 4$ apart from the point $s=\varphi_{0}$, where it has a simple pole with the residue defined by the incident field (2.1)

$$
\underset{s=\varphi_{0}}{\operatorname{res}} \mathcal{S}_{1}(s)=1
$$

The second spectral function $\mathcal{S}_{2}(s)$ is analytic in the strip $|\operatorname{Re} s|<\pi / 4$ and continuous everywhere in the strip $|\operatorname{Re} s| \leqslant \pi / 4$. At infinity, as $\operatorname{Im} s \rightarrow \pm \infty$ and $\operatorname{Re} s$ is finite, both functions are at most bounded: $\left|\mathcal{S}_{j}(s)\right| \leqslant$ const, $j=1,2$.

\section{Derivation of a functional-difference equation}

The symmetry of the contour $\Gamma$ and the boundary conditions (2.2), (2.5) imply (Maliuzhinets (2))

$$
\begin{aligned}
(\sin s-\gamma)\left[\mathcal{S}_{1}\left(s+\frac{3 \pi}{4}\right)+\mathcal{S}_{2}\left(-s-\frac{\pi}{4}\right)\right] & =-(\sin s+\gamma)\left[\mathcal{S}_{1}\left(-s+\frac{3 \pi}{4}\right)+\mathcal{S}_{2}\left(s-\frac{\pi}{4}\right)\right] \\
\mathcal{S}_{1}\left(s+\frac{3 \pi}{4}\right)-\mathcal{S}_{2}\left(-s-\frac{\pi}{4}\right) & =-\mathcal{S}_{1}\left(-s+\frac{3 \pi}{4}\right)+\mathcal{S}_{2}\left(s-\frac{\pi}{4}\right) \\
\mathcal{S}_{1}\left(s-\frac{3 \pi}{4}\right) & =\mathcal{S}_{1}\left(-s-\frac{3 \pi}{4}\right) \\
\mathcal{S}_{2}\left(s+\frac{\pi}{4}\right) & =\mathcal{S}_{2}\left(-s+\frac{\pi}{4}\right)
\end{aligned}
$$

This system expresses the function $\mathcal{S}_{2}$ through the function $\mathcal{S}_{1}$ :

$$
\mathcal{S}_{2}(s)=\left(1-\frac{\sin (s+\pi / 4)}{\sin \theta}\right) \mathcal{S}_{1}(s+\pi)-\frac{\sin (s+\pi / 4)}{\sin \theta} \mathcal{S}_{1}(s-2 \pi) .
$$

The spectral function $\mathcal{S}_{1}$ is a solution to the functional-difference equation

$$
(\sin s+\sin \theta)\left[\mathcal{S}_{1}\left(s+\frac{7 \pi}{4}\right)-\mathcal{S}_{1}\left(s-\frac{9 \pi}{4}\right)\right]=\sin s\left[\mathcal{S}_{1}\left(s+\frac{3 \pi}{4}\right)-\mathcal{S}_{1}\left(s-\frac{5 \pi}{4}\right)\right]
$$


The usual trick, found in Maliuzhinets (2), of eliminating the pole at $s=\varphi_{0}$ is to split the function $\mathcal{S}_{1}(s)$ into two factors

$$
\mathcal{S}_{1}(s)=\Sigma(s) \psi\left(s+\frac{3 \pi}{4}\right)
$$

where

$$
\Sigma(s)=\frac{2 \cos 2 \varphi_{0}}{\sin 2 s-\sin 2 \varphi_{0}} .
$$

It may be noted that the function $\psi(s)$ is analytic everywhere in the strip $|\operatorname{Re} s|<3 \pi / 2$ and also it is even. To simplify equation (3.3) introduce the new function

$$
f(s)=\psi(s+\pi)-\psi(s-\pi),
$$

which is a solution to the following problem.

Find an odd function $f(s)$ meromorphic in the $s$-plane, free of poles in the strip $|\operatorname{Re} s| \leqslant \pi / 2$ and satisfying the functional-difference equation

$$
(\cos s-\sin \theta)[f(s+\pi)+f(s-\pi)]=\cos s f(s) .
$$

At infinity, the function $f(s)$ may grow:

$$
|f(s)| \leqslant C e^{2|\operatorname{Im} s|}, \quad \operatorname{Im} s \rightarrow \infty, \quad \operatorname{Re} s \text { is finite, } \quad C=\text { const. }
$$

Analysis of equation (3.7) shows that the function $f(s)$ can have simple poles at the zeros of the function $\cos s+\sin \theta$, that is, at the points $\pm(\pi / 2+\theta)+2 \pi n(n \in \mathbb{Z})$. As for the zeros of the function $\cos s$, the points $s=\pi / 2+\pi n(n \in \mathbb{Z})$, they cannot be poles of the function $f(s)$. Indeed, the function $f(s)$ is analytic in the strip $-\pi / 2 \leqslant \operatorname{Re} s \leqslant \pi / 2$. Therefore

$$
\underset{s= \pm \pi / 2}{\operatorname{res}} f(s)=0 \text {. }
$$

This requirement and equation (3.7) at the points $\pm \pi / 2$ yield $f( \pm 3 \pi / 2)=-f(\mp \pi / 2)$, that is, the points $s= \pm 3 \pi / 2$ are removable points. By the same argument the function $f(s)$ is bounded at the other points $\pm 5 \pi / 2, \pm 7 \pi / 2, \ldots$.

For the method to be used, it is vital to have the shift in a functional-difference equation to be not less than the period of the coefficients. In the case under consideration the shift and the period are equal to $\pi$ and $2 \pi$, respectively. To transform equation (3.7) into the desired form, we replace $s$ in (3.7) first by $s+\pi$ and then by $s-\pi$. Afterwards, we eliminate the terms $f(s \pm \pi)$ from the two new equations. Finally, we obtain the following functional-difference equation

$$
a(s)[f(s+2 \pi)+f(s-2 \pi)]+b(s) f(s)=0,
$$

where

$$
a(s)=\cos ^{2} s-\sin ^{2} \theta, \quad b(s)=\cos ^{2} s-2 \sin ^{2} \theta .
$$


Now the coefficients are $\pi$-periodic functions, and the shift equals $2 \pi$. Clearly, if the function $f(s)$ is a solution of equation (3.7), then it satisfies the auxiliary equation (3.10). However, generally, the inverse statement is not correct. In sections 4 and 5 we construct the general solution of the auxiliary equation and show what to do with it in order to find the general solution of the main equation (3.7).

Solving the functional-difference equation (3.7) is crucial for derivation of the spectral functions $\mathcal{S}_{1}(s)$ and $\mathcal{S}_{2}(s)$. Assume the function $f(s)$ has been found. Then the general even solution to equation (3.6) growing at infinity as $e^{|2 \operatorname{Im} s|}$ becomes

$$
\begin{gathered}
\psi(s)=C_{0}+C_{1} \cos s+C_{2} \cos 2 s+\frac{\cos s}{4 \pi i} \int_{\Omega} \Lambda(\tau, s) f(\tau-\pi) d \tau, \quad-\pi<\operatorname{Re} s<\pi, \\
\psi(\sigma \pm \pi)=C_{0}-C_{1} \cos \sigma+C_{2} \cos 2 \sigma \pm \frac{f(\sigma)}{2}-\frac{\cos \sigma}{4 \pi i} \int_{\Omega} \Lambda(\tau, \sigma+\pi) f(\tau-\pi) d \tau, \quad \operatorname{Re} \sigma=0
\end{gathered}
$$

where

$$
\Lambda(\tau, s)=\left(\cot \frac{\tau-s}{2}-\cot \frac{\tau-s_{0}}{2}\right) \frac{1}{\cos \tau},
$$

$\Omega=\{s \in \mathbb{C}: \operatorname{Re} s=\pi\}$, and $s_{0}$ is an arbitrary fixed internal point in the strip $\Pi=\{s \in \mathbb{C}:-\pi<$ $\operatorname{Re} s<\pi\}$ such that $\operatorname{Re} s_{0} \neq 0$. Relations (3.13) are the Sokhotski-Plemelj formulae for the integral (3.12) with the periodic analogue (3.14) of the Cauchy kernel. The choice of the kernel ensures the convergence of the integrals (3.12), (3.13) with the density satisfying the inequality (3.8). The integral in (3.13) is understood in the sense of the principal value. The constants $C_{0}$ and $C_{1}$ are arbitrary. We next show that the constant $C_{2}$ is zero. Indeed, if $C_{2} \neq 0$, then $\psi(s) \sim C_{2} \cos 2 s$, $\operatorname{Im} s \rightarrow \infty$, and therefore by (3.2), (3.4) and (3.5),

$$
S_{2}(s) \sim-\frac{4 \cos 2 \varphi_{0}}{\sin \theta} C_{2} \sin \left(s+\frac{\pi}{4}\right), \quad \operatorname{Im} s \rightarrow \infty .
$$

This means that $S_{2}(s)$ has an exponential growth at infinity that is not acceptable. So $C_{2}=0$.

Outside the strip $-\pi \leqslant \operatorname{Re} s \leqslant \pi$, the function $\psi(s)$ is defined by analytical continuation. For example

$$
\begin{gathered}
\psi(s)=f(s-\pi)+\psi(s-2 \pi), \quad \pi \leqslant \operatorname{Re} s \leqslant 2 \pi, \\
\psi(s)=-f(s+\pi)+\psi(s+2 \pi), \quad-2 \pi \leqslant \operatorname{Re} s \leqslant-\pi .
\end{gathered}
$$

Now express the spectral functions $\mathcal{S}_{1}(s), \mathcal{S}_{2}(s)$ in terms of the function $\psi(s)$ and the solution to the functional-difference equation (3.7), the function $f(s)$. From (3.4) and (3.16) we derive

$$
\begin{gathered}
\mathcal{S}_{1}(s)=\Sigma(s) \psi\left(s+\frac{3 \pi}{4}\right), \quad-\frac{7 \pi}{4} \leqslant \operatorname{Re} s \leqslant \frac{\pi}{4}, \\
\mathcal{S}_{1}(s)=\Sigma(s)\left[\psi\left(s-\frac{5 \pi}{4}\right)+f\left(s-\frac{\pi}{4}\right)\right], \quad \frac{\pi}{4} \leqslant \operatorname{Re} s \leqslant \frac{5 \pi}{4} .
\end{gathered}
$$

The relations (3.2) and (3.16) give

$$
\begin{aligned}
\mathcal{S}_{2}(s)= & \Sigma(s)\left\{\left(1-\frac{\sin (s+\pi / 4)}{\sin \theta}\right)\left[\psi\left(s-\frac{\pi}{4}\right)+f\left(s+\frac{3 \pi}{4}\right)\right]\right. \\
& \left.-\frac{\sin (s+\pi / 4)}{\sin \theta}\left[\psi\left(s+\frac{3 \pi}{4}\right)-f\left(s-\frac{\pi}{4}\right)\right]\right\}, \quad-\frac{3 \pi}{4} \leqslant \operatorname{Re} s \leqslant \frac{\pi}{4} .
\end{aligned}
$$


Analysis of the last formula indicates that if the function $f(s)$ has a pole at the point $s=\pi / 2+\theta$, then the spectral function $\mathcal{S}_{2}(s)$ has an inadmissible pole at the point $s=-\pi / 4+\theta$ in the strip $-\pi / 4<\operatorname{Re} s<\pi / 4$. Therefore, because the function $f(s)$ is odd, $s= \pm(\pi / 2+\theta)$ have to be removable points:

$$
\underset{s= \pm(\pi / 2+\theta)}{\operatorname{res}} f(s)=0
$$

\section{General solution of the auxiliary functional-difference equation (3.10)}

\subsection{Derivation of a scalar Riemann-Hilbert problem on an elliptic surface}

In this section we reduce the functional-difference equation (3.10) first to a vector Riemann-Hilbert boundary-value problem on a complex plane and then to a scalar problem on an elliptic surface. Introduce two functions

$$
\Phi_{1}(s)=f(s), \quad \Phi_{2}(s)=f(s+2 \pi), \quad s \in \bar{\Pi}=\{s \in \mathbb{C}:-\pi \leqslant \operatorname{Re} s \leqslant \pi\} .
$$

Then on the contour $\Omega, \Phi_{1}(\sigma)=\Phi_{2}(\sigma-2 \pi)$, and equation (3.10) can be written as a vector functional-difference equation of the first order

$$
\boldsymbol{\Phi}(\sigma)=\mathbf{G}(\sigma) \boldsymbol{\Phi}(\sigma-2 \pi), \quad \sigma \in \Omega,
$$

where

$$
\boldsymbol{\Phi}(s)=\left(\begin{array}{l}
\Phi_{1}(s) \\
\Phi_{2}(s)
\end{array}\right), \quad \mathbf{G}(s)=\left(\begin{array}{cc}
0 & 1 \\
-1 & -b(s) / a(s)
\end{array}\right)
$$

4.1.1 Eigenvalues of the matrix $\mathbf{G}(s)$. Analyse the eigenvalues of the matrix $\mathbf{G}(s)$

$$
\lambda_{j}(s)=\frac{-b(s)+(-1)^{j-1} \Delta^{1 / 2}(s)}{2 a(s)}, \quad j=1,2,
$$

where the function $\Delta(s)$ has the form

$$
\Delta(s)=b^{2}(s)-4 a^{2}(s)=\cos ^{2} s\left(4 \sin ^{2} \theta-3 \cos ^{2} s\right) .
$$

Let

$$
\eta=\arccos \left(\frac{2}{\sqrt{3}} \sin \theta\right) .
$$

Because of the assumption $0<\operatorname{Re} \theta<\pi / 2, \operatorname{Re} \sin \theta>0$, and thus $0 \leqslant \operatorname{Re} \eta<\pi / 2$. Clearly, if $\eta=0$ (this means $\sin \theta=\gamma=\frac{1}{2} \sqrt{3}>0$ ) then the function $\Delta^{1 / 2}(s)$ does not have branch points in the strip $\Pi$. This particular case is reported in section 7 . Here we assume that $\eta \neq 0$. Let

$$
\beta(s)=\sqrt{\cos ^{2} s-\cos ^{2} \eta}
$$




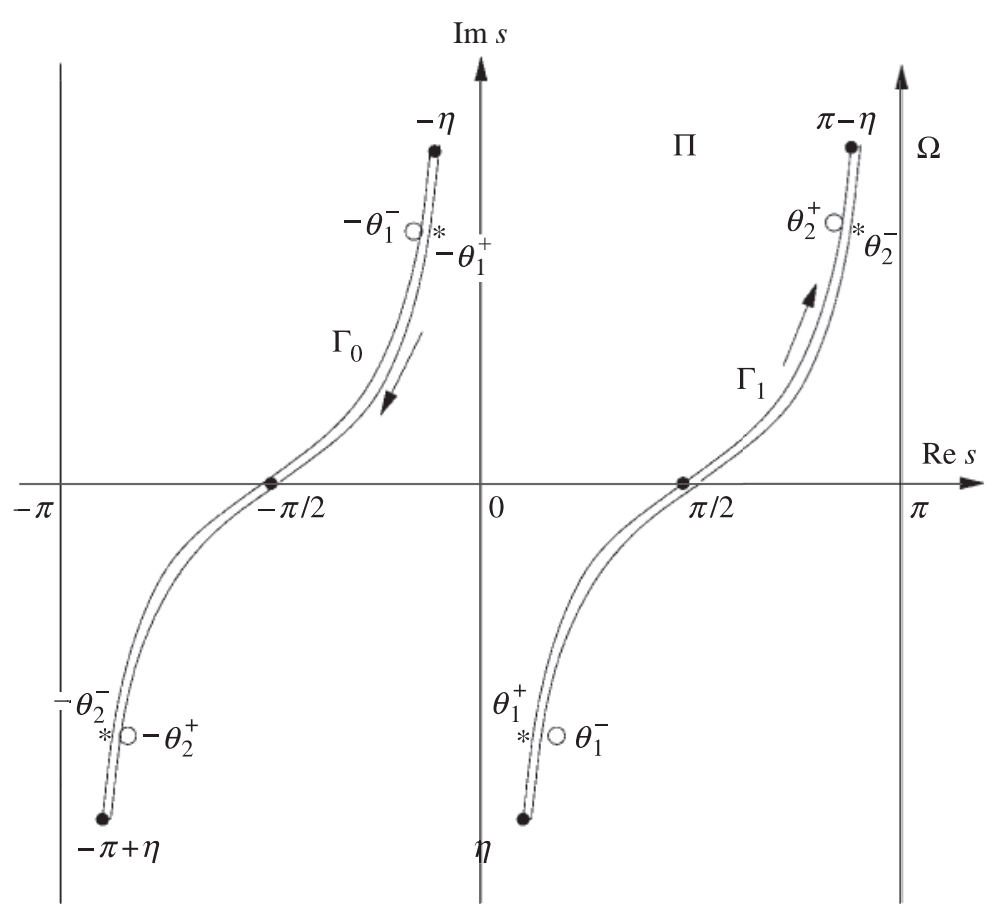

Fig. 2 The branch points and the cuts

Then $\Delta^{1 / 2}(s)=i \sqrt{3} \beta(s) \cos s$, and the eigenvalues (4.4) of the matrix $\mathbf{G}(s)$ can be written as follows:

$$
\lambda_{j}(s)=\frac{\cos s-(-1)^{j} i \sqrt{3} \beta(s)}{\cos s+(-1)^{j} i \sqrt{3} \beta(s)}, \quad j=1,2 .
$$

The function $\beta(s)$ has four branch points in the strip $\Pi$. These points are $s_{0}=-\pi+\eta, s_{1}=-\eta$, $s_{2}=\eta$ and $s_{3}=\pi-\eta$. To fix a branch of the function $\beta(s)$, cut the strip $\Pi$ along smooth curves $\Gamma_{0}, \Gamma_{1}$ joining the branch points $-\pi+\eta$ with $-\eta$ and $\eta$ with $\pi-\eta$ (Fig. 2 ). The curve $\Gamma_{1}$ is chosen such that it passes through the points $\pi / 2-\theta, \pi / 2$ and $\pi / 2+\theta$ and it is located symmetrically with respect to the point $s=\pi / 2$. The curve $\Gamma_{0}$ is obtained by reflecting the curve $\Gamma_{1}$ through the origin. This special choice of the cuts $\Gamma_{0}, \Gamma_{1}$ is required for the solution $f(s)$ to be odd and will be used later in this section. The branch curves do not intersect the contour $\Omega$. This fact is essential for the solution of equation (3.10) to be single-valued. We fix the branch $\beta(s)$ such that $\beta(0)=\sin \eta$. This branch is an even function and possesses the following properties:

$$
\begin{aligned}
& \beta(s) \sim \cos s, \quad \operatorname{Im} s \rightarrow \pm \infty, \\
& \beta(s \pm \pi)=-\beta(s), \quad s \in \Pi,
\end{aligned}
$$




$$
\beta^{+}(\sigma)=-\beta^{-}(\sigma), \quad \sigma \in \Gamma_{j}, \quad j=0,1
$$

Then $\lambda_{j}(s)$ are even single-valued analytic functions in the strip $\Pi$ cut along the curves $\Gamma_{0}, \Gamma_{1}$. On the sides of the cuts,

$$
\lambda_{j}^{+}(\sigma) \lambda_{j}^{-}(\sigma)=1, \quad \sigma \in \Gamma_{j}, \quad j=0,1 .
$$

Let $s=\eta$ and $s=-\eta$ be the initial points and $s=\pi-\eta$ and $s=-\pi+\eta$ be the terminal points of the curves $\Gamma_{1}$ and $\Gamma_{0}$, respectively. Denote by $\Gamma_{j}^{+}$the left and by $\Gamma_{j}^{-}$the right banks of the cuts $\Gamma_{j}(j=0,1)$ with respect to the positive direction. Because of the choice of the cuts the functions $1 / \lambda_{1}(s)$ and $\lambda_{1}(s)$ have zeros of the first order at the points $\theta_{1}^{+}=\pi / 2-\theta \in \Gamma_{1}^{+}$and $\theta_{2}^{+}=\pi / 2+\theta \in \Gamma_{1}^{+}$, respectively. On the right side of the cut $\Gamma_{1}$ at the corresponding opposite points $\theta_{1}^{-}=\pi / 2-\theta \in \Gamma_{1}^{-}$and $\theta_{2}^{-}=\pi / 2+\theta \in \Gamma_{1}^{-}$, the functions $\lambda_{1}(s)$ and $1 / \lambda_{1}(s)$ have first-order zeros. The zeros of the functions $1 / \lambda_{1}(s)$ and $\lambda_{1}(s)$ on the sides of the cut $\Gamma_{0}$ are defined by the symmetry. In Fig. 2 the zeros of the functions $\lambda_{1}(s)$ and $1 / \lambda_{1}(s)$ are circled and starred respectively. These properties of the eigenvalues $\lambda_{1}(s)$ and $\lambda_{2}(s)$ will be used later for solution of the Jacobi inversion problem and specification of the general solution of equation (3.7) from the solution of equation (3.10).

4.1.2 Vector Riemann-Hilbert problem on a system of curves. Next, we diagonalize the matrix $\mathbf{G}(s)$ :

$$
[\mathbf{T}(s)]^{-1} \mathbf{G}(s) \mathbf{T}(s-h)=\Lambda(s),
$$

where

$$
\mathbf{T}(s)=\left(\begin{array}{cc}
1 & 1 \\
\lambda_{1}(s) & \lambda_{2}(s)
\end{array}\right), \quad \boldsymbol{\Lambda}(s)=\left(\begin{array}{cc}
\lambda_{1}(s) & 0 \\
0 & \lambda_{2}(s)
\end{array}\right),
$$

and introduce the new vector function $\phi(s)=[\mathbf{T}(s)]^{-1} \mathbf{\Phi}(s), s \in \Pi$, with the components

$$
\begin{gathered}
\phi_{1}(s)=\frac{1}{2}\left(\frac{b(s)}{\Delta^{1 / 2}(s)}+1\right) \Phi_{1}(s)+\frac{a(s)}{\Delta^{1 / 2}} \Phi_{2}(s), \\
\phi_{2}(s)=\frac{1}{2}\left(-\frac{b(s)}{\Delta^{1 / 2}(s)}+1\right) \Phi_{1}(s)-\frac{a(s)}{\Delta^{1 / 2}(s)} \Phi_{2}(s) .
\end{gathered}
$$

Then these functions satisfy the two separate equations

$$
\phi_{j}(\sigma)=\lambda_{j}(\sigma) \phi_{j}(\sigma-2 \pi), \quad \sigma \in \Omega, \quad j=1,2 .
$$

In order that the vector function $\Phi(s)$ is single-valued in the strip $\Pi$ it is necessary and sufficient that (see (6))

$$
\phi_{1}^{+}(\sigma)=\phi_{2}^{-}(\sigma), \quad \phi_{1}^{-}(\sigma)=\phi_{2}^{+}(\sigma), \quad \sigma \in \Gamma_{j}, \quad j=0,1,
$$

where $\phi_{j}^{ \pm}(\sigma)$ are the limiting values of the functions $\phi_{j}(s)$ on the left $(+)$ and the right $(-)$ sides of the contours $\Gamma_{0}, \Gamma_{1}$. Transform now the problem (4.16), (4.17) into a vector Riemann-Hilbert problem on a system of segments. Take the mapping function $z=u(s)$ as follows:

$$
u(s)=\tan \frac{\eta}{2} \cot \frac{s}{2}
$$




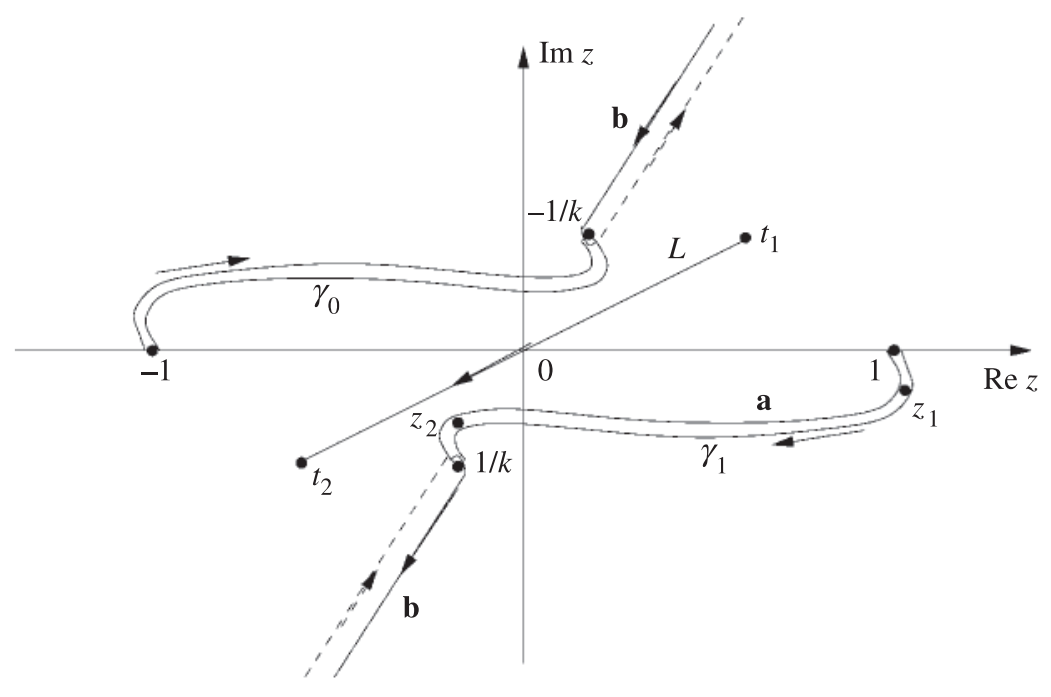

Fig. 3 The canonical cross-sections $\mathbf{a}, \mathbf{b}$ and the contour $L$

Then its inverse function $s=v(z)$ becomes

$$
v(z)=-i \log \frac{z+i \tan \frac{1}{2} \eta}{z-i \tan \frac{1}{2} \eta} .
$$

The single branch of the logarithmic function $v(z)$ is chosen such that $v(\infty)=0$. The contour $\Omega$ is mapped onto the left side of the straight segment $L$ with the starting point $t_{1}=i \tan \frac{1}{2} \eta$ and the ending point $t_{2}=-i \tan \frac{1}{2} \eta$ (Fig.3). The left boundary $\{s \in \mathbb{C}: \operatorname{Re} s=-\pi\}$ of the strip $\Pi$ is mapped onto the right side of the contour $L$. As for the branch points $s_{0}, s_{1}, s_{2}$ and $s_{3}$, they fall into the points $-1 / k,-1,1$ and $1 / k$, where $k=\cot ^{2} \frac{1}{2} \eta$. The cuts $\Gamma_{0}, \Gamma_{1}$ become smooth curves $\gamma_{0}, \gamma_{1}$ joining the points $-1 / k,-1$ and $1,1 / k$. The curves $\gamma_{0}, \gamma_{1}$ do not cross the contour $L$.

We write down the vector Riemann-Hilbert problem that is equivalent to equations (4.16), (4.17):

$$
\begin{gathered}
F_{j}^{+}(t)=l_{j}(t) F_{j}^{-}(t), \quad t \in L, \quad j=1,2, \\
F_{1}^{+}(t)=F_{2}^{-}(t), \quad F_{1}^{-}(t)=F_{2}^{+}(t), \quad t \in \gamma_{m}, \quad m=0,1,
\end{gathered}
$$

where

$$
\begin{gathered}
F_{j}^{ \pm}(t)=\phi_{j}^{ \pm}(\sigma), \quad l_{j}(t)=\lambda_{j}(\sigma), \\
\sigma=-i \log \frac{t+i \tan \frac{1}{2} \eta}{t-i \tan \frac{1}{2} \eta}, \quad j=1,2 .
\end{gathered}
$$


4.1.3 Scalar Riemann-Hilbert problem on an elliptic surface. To solve the vector problem (4.20), (4.21), convert it into a scalar Riemann-Hilbert problem on a Riemann surface. Let $\mathcal{R}$ be the elliptic surface of the algebraic function

$$
w^{2}=q(z), \quad q(z)=\left(1-z^{2}\right)\left(1-k^{2} z^{2}\right),
$$

formed by gluing two copies $\mathbb{C}_{1}$ and $\mathbb{C}_{2}$ of the extended complex plane $\mathbb{C} \cup \infty$ cut along the curves $\gamma_{0}$ and $\gamma_{1}$. The positive (left) sides of the cuts $\gamma_{m}(m=0,1)$ on $\mathbb{C}_{1}$ are glued to the negative (right) sides of the curves $\gamma_{m}$ on $\mathbb{C}_{2}$ and vice versa.

Let $q^{1 / 2}(z)$ be the branch chosen such that $q^{1 / 2}(0)=1$. Then the function $w$ defined by (4.23) is single-valued on the surface $\mathcal{R}: w=q^{1 / 2}(z), z \in \mathbb{C}_{1}$. Introduce now the following functions on the surface $\mathcal{R}$ :

$$
F(z, w)=\left\{\begin{array}{ll}
F_{1}(z), & (z, w) \in \mathbb{C}_{1}, \\
F_{2}(z), & (z, w) \in \mathbb{C}_{2},
\end{array} \quad l(t, \xi)= \begin{cases}l_{1}(t), & (t, \xi) \in L_{1}, \\
l_{2}(t), & (t, \xi) \in L_{2}\end{cases}\right.
$$

From (4.21) it becomes evident that the function $F(z, w)$ is meromorphic everywhere on the surface $\mathcal{R}$ apart from the contour $\mathcal{L}=L_{1} \cup L_{2}$, with $L_{1}=L \subset \mathbb{C}_{1}$ and $L_{2}=L \subset \mathbb{C}_{2}$. On the contour $\mathcal{L} \subset \mathcal{R}$, this function satisfies the boundary condition

$$
F^{+}(t, \xi)=l(t, \xi) F^{-}(t, \xi), \quad(t, \xi) \in \mathcal{L},
$$

where $\xi=w(t)$. At the end points the function $F(z, w)$ has the following asymptotic behaviour:

$$
|F(z, w)| \leqslant A_{j}\left|z-t_{j}\right|^{-2}, \quad(z, w) \in \mathcal{R}, \quad z \rightarrow t_{j}, \quad j=1,2, \quad A_{j}=\text { const. }
$$

\subsection{Factorization of the function $l(t, \xi)$}

To solve the Riemann-Hilbert problem (4.25) one needs to factorize the coefficient $l(t, \xi)$. This means constructing a canonical solution to the problem $(4.25)$, that is, a function $X(z, w)$ meromorphic on $\mathcal{R} \backslash \mathcal{L}$ with at most a finite number of poles and zeros on $\mathcal{R}$ such that its boundary values $X^{ \pm}(t, \xi)$ are non-zero, and that $(t, \xi) \in \mathcal{L} \subset \mathcal{R}$ and satisfy the boundary condition

$$
X^{+}(t, \xi)=l(t, \xi) X^{-}(t, \xi), \quad(t, \xi) \in \mathcal{L} \subset \mathcal{R} .
$$

At the end points $z=t_{1}$ and $z=t_{2}$ this solution is bounded:

$$
|X(z, w)| \leqslant A, \quad(z, w) \in \mathcal{R}, \quad z \rightarrow t_{j}, \quad j=1,2, \quad A=\text { const. }
$$

First, define $\mathbf{a}$ - and $\mathbf{b}$-canonical cross-sections of the surface $\mathcal{R}$. The cross-section a consists of the banks of the cut $\gamma_{1}$ (Fig. 3) which simultaneously belong to $\mathbb{C}_{1}$ and $\mathbb{C}_{2}$. The positive direction on $\mathbf{a}$ is chosen such that the first sheet $\mathbb{C}_{1}$ is always on the left. The cross-section $\mathbf{b}$ is a smooth closed curve that consists of two parts. The first part is a curve of $\mathbb{C}_{1}$ joining the points $1 / k$ and $-1 / k$ and passing through infinity. The second part lies on the sheet $\mathbb{C}_{2}$ and joins the points $-1 / k$ and $1 / k$ through infinity. The starting point is $1 / k$ and the first sheet is traced first. Both parts of the cross-section $\mathbf{b}$ are symmetric with respect to the origin.

It is directly verified that $\lambda_{j}(\sigma)=\lambda_{j}(\bar{\sigma}), \sigma \in \Omega$. Therefore, $l_{j}(t)=l_{j}(-t), t \in L, j=1,2$, and the coefficient of the problem (4.27) is even: $l(-t, \xi)=l(t, \xi),(t, \xi) \in \mathcal{L}$. To explore this property 
of the function $l(t, \xi)$, we first find a meromorphic solution of the following auxiliary problem for a half of the contour $\mathcal{L}$

$$
X_{0}^{+}(t, \xi)=l(t, \xi) X_{0}^{-}(t, \xi), \quad(t, \xi) \in \mathcal{L}^{\prime},
$$

where $\mathcal{L}^{\prime}$ consists of the two segments $L^{\prime}=\left[t_{1}, 0\right]$ lying on the two sheets $\mathbb{C}_{1}$ and $\mathbb{C}_{2}$ of the surface. We seek a solution bounded at the end point $z=t_{1}$ and do not prescribe the asymptotics of the solution at the point $z=0$.

A canonical solution to the problem (4.29) is given by Antipov and Silvestrov (6, 8):

$$
\begin{gathered}
X_{0}(z, w)=\exp \{\chi(z, w)\}, \\
\chi(z, w)=\frac{1}{2 \pi i} \int_{\mathcal{L}^{\prime}} \log l(t, \xi) d W+\sum_{\mu=1}^{2} \operatorname{sgn} \kappa_{\mu} \sum_{j=1}^{\left|\kappa_{\mu}\right|} \int_{p_{\mu 0}}^{p_{\mu j}} d W \\
+\int_{\left(\delta_{0}, v_{0}\right)}^{\left(\sigma_{0}, u_{0}\right)} d W+m_{0} \oint_{\mathbf{a}} d W+n_{0} \oint_{\mathbf{b}} d W,
\end{gathered}
$$

where

$$
d W=\frac{w+\xi}{2 \xi} \frac{d t}{t-z}, \quad w=w(z), \quad \xi=w(t),
$$

is the Weierstrass kernel, an analogue of the Cauchy kernel on the surface $\mathcal{R}$. It is directly verified that

$$
l_{j}\left(t_{1}\right)=\lambda_{j}(\pi-i \infty)=-\frac{1}{2}+(-1)^{j-1} \frac{i \sqrt{3}}{2}, \quad j=1,2 .
$$

We fix the single branches of the logarithmic functions as follows:

$$
\arg l_{1}\left(t_{1}\right)=-\frac{4 \pi}{3}, \quad \arg l_{2}\left(t_{1}\right)=-\frac{2 \pi}{3} .
$$

Analysis of the Weierstrass integrals in (4.30) implies that

$$
X_{0}(z, w)=O\left\{\left(z-t_{1}\right)^{\beta_{j}}\right\}, \quad(z, w) \in \mathbb{C}_{j}, \quad z \rightarrow t_{1}, \quad j=1,2,
$$

where $\beta_{1}=\frac{2}{3}, \beta_{2}=\frac{1}{3}$. Thus, the function $X_{0}(z, w)$ is bounded at the end point $z=t_{1}$. The integers $\kappa_{\mu}(\mu=1,2)$ are used $(\mathbf{8})$ to achieve the prescribed behaviour of the solution at the second end point, $z=0$. Since we have not fixed the asymptotics of the solution at this point, it is possible to choose the integers $\kappa_{1}$ and $\kappa_{2}$ in an arbitrary way. The simplest choice is $\kappa_{1}=\kappa_{2}=0$. Then the second term in (4.30) vanishes. The final formula for the function $F(z, w)$ is independent of $\kappa_{1}$ and $\kappa_{2}$. The point $\left(\delta_{0}, v_{0}\right) \in \mathbb{C}_{1}, v_{0}=q^{1 / 2}\left(\delta_{0}\right)$ is arbitrarily fixed. The point $\left(\sigma_{0}, u_{0}\right)\left(u_{0}=w\left(\sigma_{0}\right)\right)$ and the integers $m_{0}, n_{0}$ are not arbitrary and will be fixed later.

The second integral in (4.30) is taken over a smooth curve which joins the end points $\left(\delta_{0}, v_{0}\right)$ and $\left(\sigma_{0}, u_{0}\right)$ and which does not intersect the cross-sections $\mathbf{a}, \mathbf{b}$ and the contour $\mathcal{L}^{\prime}$. This integral is independent of the shape of the integration path. The first integral in (4.30) is discontinuous through the contour $\mathcal{L}^{\prime}$ with the jump $\log l(t, \xi)$. The other integrals are also discontinuous through the contours of integration. The corresponding jumps are equal to $2 \pi i m$ ( $m$ is an integer). Hence the function $X_{0}(z, w)$ satisfies the homogeneous boundary condition (4.29). 


\subsection{Elimination of an essential singularity at infinity}

4.3.1 Jacobi's inversion problem. In general, for an arbitrary point $\left(\sigma_{0}, u_{0}\right)$ and arbitrary integers $m_{0}, n_{0}$, the function $X_{0}(z, w)$ in (4.30) has an essential singularity at infinity. This is because $w(z) \sim(-1)^{j} k z^{2}, z \rightarrow \infty, z \in \mathbb{C}_{j}$, and the Weierstrass kernel (4.31) has a pole at infinity. To eliminate the essential singularity we evaluate the principal terms of the expansions of the function $\chi(z, w)$ at infinity on both sheets of the surface:

$$
\frac{k z}{2}(-1)^{j-1}\left\{\frac{1}{2 \pi i} \int_{L^{\prime}}\left[\log l_{1}(t)-\log l_{2}(t)\right] \frac{d t}{q^{1 / 2}(t)}+\int_{\left(\delta_{0}, v_{0}\right)}^{\left(\sigma_{0}, u_{0}\right)} \frac{d t}{\xi(t)}+m_{0} \oint_{\mathbf{a}} \frac{d t}{\xi(t)}+n_{0} \oint_{\mathbf{b}} \frac{d t}{\xi(t)}\right\}
$$

Thus in order that the function $X_{0}(z, w)$ is bounded at infinity it is necessary and sufficient that

$$
\int_{\left(\delta_{0}, v_{0}\right)}^{\left(\sigma_{0}, u_{0}\right)} \frac{d t}{\xi(t)}+m_{0} \oint_{\mathbf{a}} \frac{d t}{\xi(t)}+n_{0} \oint_{\mathbf{b}} \frac{d t}{\xi(t)}=d^{\circ}
$$

where

$$
d^{\circ}=\frac{1}{2 \pi i} \int_{L^{\prime}}\left[\log l_{2}(t)-\log l_{1}(t)\right] \frac{d t}{q^{1 / 2}(t)} .
$$

This nonlinear equation is the Jacobi inversion problem for the surface $\mathcal{R}$ of genus $\rho=1$. We next solve this problem in closed form.

4.3.2 Evaluation of the constant $d^{\circ}$. To find the solution of the problem (4.36) explicitly we simplify the expression (4.37) for the constant $d^{\circ}$. The procedure consists of two steps. At the first stage we transform the integral (4.37) into an integral over an infinite line. The second step is to evaluate this integral by non-trivial application of the Cauchy theorem.

It follows from (4.8) that $l_{1}(t) l_{2}(t)=1$. Since in addition $l_{j}(t)=l_{j}(-t)$, for the chosen branches of the functions $\log l_{j}(t)$ we establish

$$
\begin{gathered}
\log l_{j}(-t)=\log l_{j}(t), \quad \log l_{1}(t)+\log l_{2}(t)=-2 \pi i, \\
\log l_{1}(t)-\log l_{2}(t)=2 \log l_{1}(t)+2 \pi i
\end{gathered}
$$

Therefore

$$
d^{\circ}=-\frac{1}{\pi i} \int_{L^{\prime}} \frac{\log l_{1}(t)+\pi i}{q^{1 / 2}(t)} d t=-\frac{1}{2 \pi i} \int_{L} \frac{\log l_{1}(t)+\pi i}{q^{1 / 2}(t)} d t .
$$

On making substitution $t=-1 /(k \tau)$, we establish the following identities:

$$
\log l_{1}(t)=\log l_{1}(\tau), \quad q^{1 / 2}(t)=-\frac{q^{1 / 2}(\tau)}{k \tau^{2}}, \quad t=-\frac{1}{k \tau} .
$$

The second formula in (4.40) is verified directly. Show the validity of the first identity. Assume that the image (4.18) of a point $\sigma$ is $t \in L$. Then the point $\tau$ corresponds to the point $\sigma-\pi$. Because of 


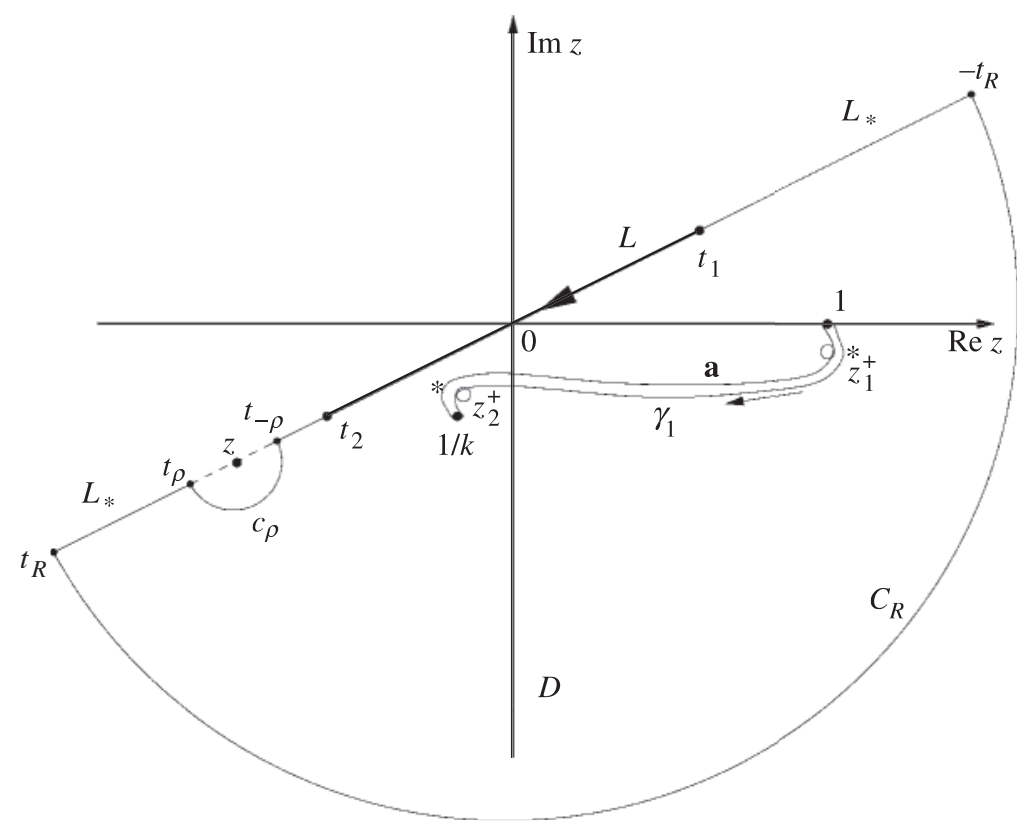

Fig. 4 The domain $D$ and the contour of integration: $\eta \neq 0$

the relations $\log l_{1}(t)=\log \lambda_{1}(\sigma)$ and $\log l_{1}(\tau)=\log \lambda_{1}(\sigma-\pi)$ and also since the image (4.18) of the point $t=t_{1}$ coincides with this point, $\tau=t_{1}$, the first formula in (4.40) follows.

The substitution $t=-1 /(k \tau)$ transforms (4.39) into the integral over two semi-infinite segments $L_{*}$ which expand the segment $\left[t_{1}, t_{2}\right]$ in both directions

$$
d^{\circ}=-\frac{1}{2 \pi i} \int_{L_{*}} \frac{\log l_{1}(\tau)+\pi i}{q^{1 / 2}(\tau)} d \tau .
$$

The positive direction on the contour $L_{*}$ is from $t_{2}$ to the point at infinity and then to the point $t_{1}$. By adding the two relations (4.39) and (4.41) we obtain

$$
2 d^{\circ}=-\frac{1}{2 \pi i} \int_{L \cup L_{*}} \frac{\log l_{1}(t)+\pi i}{q^{1 / 2}(t)} d t .
$$

By the Cauchy theorem applied to the function

$$
g(z)=\frac{\log l_{1}(z)+\pi i}{q^{1 / 2}(z)}
$$

which is holomorphic in the domain $D$ with $\rho=0$ (Fig.4), this integral can be written as follows:

$$
d^{\circ}=\frac{1}{4 \pi i} \oint_{\mathbf{a}} \frac{\log l_{1}(z)+\pi i}{q^{1 / 2}(z)} d z=\frac{1}{4 \pi i} \int_{1}^{1 / k} \frac{\log l_{1}^{+}(t)+\log l_{1}^{-}(t)+2 \pi i}{q^{1 / 2}\left(t^{+}\right)} d t .
$$


We notice that the half-plane located to the left from the contour $L \cup L_{*}$ is the image $z=u(s)$ of the strip $0<\operatorname{Re} s<\pi$. Here $t^{+}$is a point on the left bank of the cut $\gamma_{1} ; l_{1}^{+}(t)$ and $l_{1}^{-}(t)$ are the limiting values of the function $l_{1}(z)$ on the left $\gamma_{1}^{+}$and right $\gamma_{1}^{-}$banks of the cut $\gamma_{1}$. Let $z_{1}^{ \pm} \in \gamma_{1}^{ \pm}$ and $z_{2}^{ \pm} \in \gamma_{1}^{ \pm}$be the images (4.18) of the points $\theta_{1}^{ \pm}$and $\theta_{2}^{ \pm}$and

$$
z_{1}=\tan \frac{\eta}{2} \cot \left(\frac{\pi}{4}-\frac{\theta}{2}\right), \quad z_{2}=\tan \frac{\eta}{2} \cot \left(\frac{\pi}{4}+\frac{\theta}{2}\right) .
$$

At the points $z_{1}^{+}$and $z_{2}^{-}$the function $l_{1}(z)$ equals zero. At the other two points $z_{1}^{-}$and $z_{2}^{+}$it equals infinity. Therefore, the function $g(z)$ has logarithmic singularities at the points $z_{1}^{ \pm}, z_{2}^{ \pm}$. Apart from these points, the function $g(z)$ is continuous everywhere on the boundary $D$. We also notice that $|g(z)| \leqslant$ const $|z|^{-2}, z \rightarrow \infty$. This justifies the application of the Cauchy theorem (4.44) to the function $g(z)$.

We next prove the following property of the functions $l_{1}^{ \pm}(t)$

$$
\log l_{1}^{+}(t)+\log l_{1}^{-}(t)= \begin{cases}-2 \pi i, & t \in\left(z_{1}, z_{2}\right) \subset \gamma_{1}, \\ -4 \pi i, & t \in\left[1, z_{1}\right) \cup\left(z_{2}, 1 / k\right] \subset \gamma_{1} .\end{cases}
$$

First, we note that formula (4.12) implies $l_{1}^{+}(t) l_{1}^{-}(t)=1, t \in \gamma_{1}$. Therefore, on a portion of the contour $\gamma_{1}$ say, $\left(z_{1}, z_{2}\right)$, where the function $\log l_{1}(z)$ is continuous,

$$
\log l_{1}^{+}(t)+\log l_{1}^{-}(t)=2 \pi i n, \quad\left(z_{1}, z_{2}\right) \subset \gamma_{1} .
$$

where $n$ is an integer to be defined. Clearly, when the point $z$ traverses the contours $\gamma_{1}^{+}, \gamma_{1}^{-}$in the positive direction and passes the points $z_{1}^{+}, z_{2}^{-}$(the points where the function $\log l_{1}(z)$ becomes infinite), the function $\log l_{1}(z)$ receives the increment $\pi i$. Its increment becomes $-\pi i$ when it passes the other two points $z_{1}^{-}$and $z_{2}^{+}$. Thus,

$$
\log l_{1}^{+}(t)+\log l_{1}^{-}(t)=2 \pi i(n-1), \quad t \in\left[1, z_{1}\right) \cup\left(z_{2}, 1 / k\right] \subset \gamma_{1} .
$$

To fix $n$ we first evaluate the values $\log l_{1}(z)$ at the two opposite points $(1 / \sqrt{k})^{ \pm} \in\left(z_{1}, z_{2}\right)^{ \pm} \subset \gamma_{1}^{ \pm}$. The point $t=1 / \sqrt{k} \in \gamma_{1}$ is the image of the point $s=\pi / 2 \in \Gamma_{1}$. This implies $\log l_{1}^{ \pm}(1 / \sqrt{k})=$ $\log \lambda_{1}^{ \pm}(\pi / 2)$. One may observe that

$$
\log l_{1}^{-}\left(\frac{1}{\sqrt{k}}\right)=\log \lambda_{1}\left(\frac{\pi}{2}-i \infty\right)+\left.\log \lambda_{1}\left(\frac{\pi}{2}+i y\right)\right|_{y=-\infty} ^{y=-0} .
$$

To find the increment of the function $\log \lambda_{1}(\pi / 2+i y)$ in (4.49), we evaluate the integral

$$
\begin{aligned}
\left.\log \lambda_{1}\left(\frac{\pi}{2}+i y\right)\right|_{y=-\infty} ^{y=0^{-}} & =\int_{-\infty}^{0} d \log \lambda_{1}\left(\frac{\pi}{2}+i y\right) \\
& =2 \sqrt{3} i \int_{-\infty}^{0} \frac{\cos ^{2} \eta \cosh y d y}{\left(4 \sinh ^{2} y+3 \cos ^{2} \eta\right) \sqrt{\sinh ^{2} y+\cos ^{2} \eta}}=\frac{\pi i}{3}
\end{aligned}
$$

Here we have made two substitutions. First, we put $\xi=\sinh y, \xi_{0}=\cos \eta$, and then we set $\tau=$ 
$\xi\left(\xi^{2}+\xi_{0}^{2}\right)^{-1 / 2}$. Finally, on using (4.32) and (4.49), we obtain the desired value: $\log l_{1}^{-}(1 / \sqrt{k})=$ $-\pi i$. Similarly, $\log l_{1}^{+}(1 / \sqrt{k})=-\pi i$. Hence

$$
\log l_{1}^{+}(t)+\log l_{1}^{-}(t)=-2 \pi i, \quad t=1 / \sqrt{k} \in\left(z_{1}, z_{2}\right) \subset \gamma_{1} .
$$

On comparing the last formula with (4.47), $n=-1$. The relation (4.46) is proved.

By making the substitution $t=1 /(k \tau)$ and using the relation (4.46) and

$$
z_{1} z_{2}=\frac{1}{k}, \quad \frac{d t}{q^{1 / 2}\left(t^{+}\right)}=-\frac{d \tau}{q^{1 / 2}\left(\tau^{+}\right)},
$$

we can further simplify the integral (4.44)

$$
d^{\circ}=-\int_{1}^{z_{1}} \frac{d t}{q^{1 / 2}\left(t^{+}\right)}=\int_{1}^{z_{1}} \frac{d t}{q^{1 / 2}\left(t^{-}\right)} .
$$

4.3.3 Solution to the Jacobi problem. On exploring the new expression for the constant $d^{\circ}$, we find it is a straightforward matter to write down a solution of the Jacobi problem (4.36) in explicit form. Replace $d^{\circ}$ by (4.53) in equation (4.36)

$$
\int_{\left(\delta_{0}, v_{0}\right)}^{\left(\sigma_{0}, u_{0}\right)} \frac{d t}{\xi(t)}+m_{0} \oint_{\mathbf{a}} \frac{d t}{\xi(t)}+n_{0} \oint_{\mathbf{b}} \frac{d t}{\xi(t)}=\int_{1}^{z_{1}} \frac{d t}{q^{1 / 2}\left(t^{-}\right)}
$$

We observe that the above condition is identically satisfied if the points $\left(\delta_{0}, v_{0}\right),\left(\sigma_{0}, u_{0}\right)$ and the integers $m_{0}, n_{0}$ are chosen as follows:

$$
\left(\delta_{0}, v_{0}\right)=(1,0), \quad\left(\sigma_{0}, u_{0}\right)=\left(z_{1}^{-}, q^{1 / 2}\left(z_{1}^{-}\right)\right) \in \gamma_{1}^{-}, \quad m_{0}=n_{0}=0 .
$$

Under such a choice of the points and the integers, the function $X_{0}(z, w)$ in $(4.30)$ is bounded at infinity and has the form

$$
X_{0}(z, w)=\exp \left\{\frac{1}{2 \pi i} \int_{\mathcal{L}^{\prime}} \log l(t, \xi) d W+\int_{(0,1)}^{\left(\sigma_{0}, u_{0}\right)} d W\right\} .
$$

\subsection{General solution to the Riemann-Hilbert problem}

To study the behaviour at the end points of the canonical solution of the auxiliary problem (4.29) we rewrite formula (4.56) as follows:

$$
X_{0}(z, w)=\exp \left\{\chi_{10}(z)+w(z) \chi_{20}(z)\right\}
$$

where

$$
\begin{gathered}
\chi_{10}(z)=\frac{1}{2} \log \frac{\left(z-t_{1}\right)\left(z-z_{1}\right)}{z(z-1)}, \\
\chi_{20}(z)=-\frac{1}{2 \pi i} \int_{0}^{t_{1}} \frac{\left[\log l_{1}(t)+\pi i\right] d t}{q^{1 / 2}(t)(t-z)}-\frac{1}{2} \int_{1}^{z_{1}} \frac{d t}{q^{1 / 2}\left(t^{+}\right)(t-z)} .
\end{gathered}
$$


At the point $z=t_{1}$ this solution is bounded. In the vicinity of the point $z=0$ it behaves as follows:

$$
X_{0}(z, w)=z^{-1 / 2+(-1)^{j} v_{0}} X_{*}(z, w), \quad z \rightarrow 0, \quad(z, w) \in \mathbb{C}_{j}, \quad j=1,2,
$$

where $v_{0}=\left[\log l_{1}(0)+\pi i\right] /(2 \pi i)$ and $X_{*}(z, w)$ is a bounded function as $z \rightarrow 0$.

On using the condition (4.29) and also the identity $l(-t,-w)=1 / l(t, w),(t, w) \in \mathcal{L}$, that can be derived from $l_{1}(t) l_{2}(t)=1$ and $l_{j}(t)=l_{j}(-t), j=1,2$, one may show that the function $X_{1}(z, w)=X_{0}(-z,-w)$ provides a canonical solution of another auxiliary problem on the second half of the contour $\mathcal{L}$ :

$$
X_{1}^{+}(t, \xi)=l(t, \xi) X_{1}^{-}(t, \xi), \quad(t, \xi) \in \mathcal{L} \backslash \mathcal{L}^{\prime}
$$

At the point $z=-t_{1}=t_{2}$ it is bounded and in the vicinity of the point $z=0$ it has the representation

$$
X_{0}(z, w)=z^{-1 / 2-(-1)^{j} v_{0}} X_{*}(-z,-w), \quad z \rightarrow 0, \quad(z, w) \in \mathbb{C}_{j}, \quad j=1,2 .
$$

From the boundary conditions (4.29), (4.60) and also from relations (4.59) and (4.61) we deduce that the function $X(z, w)=z X_{0}(z, w) X_{1}(z, w)$ is the canonical solution for the problem (4.27). This solution is bounded not only at the ends of the contour $\mathcal{L}$ but also at the point $z=0$. Finally, after straightforward transformations we obtain

$$
X(z, w)=\chi_{3}(z) \exp \left\{w(z) \chi_{2}(z)\right\}
$$

where

$$
\begin{gathered}
\chi_{2}(z)=-\frac{z}{\pi i} \int_{0}^{t_{1}} \frac{\left[\log l_{1}(t)+\pi i\right] d t}{q^{1 / 2}(t)\left(t^{2}-z^{2}\right)}-z \int_{1}^{z_{1}} \frac{d t}{q^{1 / 2}\left(t^{+}\right)\left(t^{2}-z^{2}\right)}, \\
\chi_{3}(z)=\sqrt{\frac{\left(z^{2}-t_{1}^{2}\right)\left(z^{2}-z_{1}^{2}\right)}{z^{2}-1}}, \quad t_{1}=i \tan \frac{\eta}{2}, \quad z_{1}=\tan \frac{\eta}{2} \cot \left(\frac{\pi}{4}-\frac{\theta}{2}\right) .
\end{gathered}
$$

A single-valued branch of the function $\chi_{3}(z)$ is chosen such that $\chi_{3}(z) \sim z, z \rightarrow \infty$. The first integral is taken over the segment $\left[0, t_{1}\right]$. The contour of integration for the second integral is the portion of the positive bank of the cut $\gamma_{1}$ with the starting point $z=1$ and the terminal point $z=z_{1}$. Both functions $\chi_{2}(z)$ and $\chi_{3}(z)$ are odd holomorphic functions in the $z$-plane with the cuts along the line $L$ and the curves $\left(-z_{1},-1\right) \subset \gamma_{0},\left(1, z_{1}\right) \subset \gamma_{1}$.

Now we can use the above formula for the canonical function $X(z, w)$ to write down the general solution of the auxiliary Riemann-Hilbert problem (4.27), (4.28):

$$
F(z, w)=X(z, w)\left[R_{1}(z)+w(z) R_{2}(z)\right],
$$

where $R_{1}(z), R_{2}(z)$ are arbitrary rational functions with zeros and poles defined by the properties of the functions $F(z, w)$ and $X(z, w)$. These functions for the problem of interest (3.7) will be derived in section 5 .

\subsection{The function $f(s)$}

Finally, we find the general odd solution of the auxiliary equation (3.10). According to formulae (4.1), (4.15), (4.22) and (4.24) the general solution of equation (3.10) in the strip $\bar{\Pi}:-\pi \leqslant \operatorname{Re} s \leqslant$ $\pi$ is given by

$$
f(s)=\Phi_{1}(s)=\phi_{1}(s)+\phi_{2}(s)=F_{1}(z)+F_{2}(z)=F(z, w)+F(z,-w),
$$


where $w=q^{1 / 2}(z)$. By making use of formulae (4.64), (4.62) and (4.63) we transform the above formula to the form

$$
f(s)=\chi_{3}(z)\left[M_{1}(s) \cosh \chi_{4}(z)+M_{2}(s) q^{1 / 2}(z) \sinh \chi_{4}(z)\right], \quad \pi \leqslant \operatorname{Re} s \leqslant \pi,
$$

where

$$
\chi_{4}(z)=q^{1 / 2}(z) \chi_{2}(z), \quad z=\tan \frac{\eta}{2} \cot \frac{s}{2} .
$$

The functions $M_{j}(s)=2 R_{j}(z), j=1,2$, are arbitrary $2 \pi$-periodic meromorphic functions. Their zeros and poles are defined by the properties of the unknown function $f(s)$ and the known functions $q^{1 / 2}(z), \chi_{3}(z), \chi_{4}(z)$. The functions $\chi_{3}(z)$ and $\chi_{4}(z)$ are odd and the function $q^{1 / 2}(z)$ is even. Therefore, for the general solution of equation (3.10) $f(s)$ to be odd, it is necessary and sufficient that the function $M_{1}(s)$ is even and the function $M_{2}(s)$ is odd.

In what follows, we show how to select the general odd solution of equation (3.7) from the family of solutions given by (4.66). To do this it is sufficient to know the general solution of equation (3.10) in the strip $-\pi \leqslant \operatorname{Re} s \leqslant \pi$ only. However, we write down the solution in the next strip $\pi \leqslant \operatorname{Re} s \leqslant 3 \pi$ in case its necessity might arise in other applications. So, if $\pi \leqslant \operatorname{Re} s \leqslant 3 \pi$, then

$$
f(s)=-\frac{b(s)}{2 a(s)}[F(z, w)+F(z,-w)]+\frac{\Delta^{1 / 2}(s)}{2 a(s)}[F(z, w)-F(z,-w)] .
$$

Analytical continuation of the solution for the whole $s$-pane can be derived by equation (3.10). The function $f(s)$ is meromorphic and single-valued in the $s$-plane.

\section{Solution of the governing functional-difference equation (3.7)}

This section presents a procedure for constructing the general odd solution of a second-order difference equation when the shift is less than the period of the coefficients involved. We exemplify the technique by solving equation (3.7). The method picks up those functions from the general solution of equation (3.10) which satisfies the equation (3.7). To achieve this goal we first assume that $s$ is any point of the imaginary axis. Next, we substitute the general solution of the auxiliary equation (3.10) into equation (3.7) and establish conditions for the arbitrary $2 \pi$-periodic meromorphic functions $M_{1}(s)$ and $M_{2}(s)$, in order that the solution of equation (3.10) satisfies equation (3.7). Thirdly, by the uniqueness of analytical continuation we find the most general form of the meromorphic solution of the equation of interest. Finally, by requiring for the solution to be within the prescribed class we find the functions $M_{1}(s)$ and $M_{2}(s)$.

\subsection{General meromorphic solution}

Let $s$ be a point of the imaginary axis. To simplify the expressions for $f(s \pm \pi)$ we notice that the image (4.18) of the point $s$ is the point $z=\tan \frac{1}{2} \eta \cot \frac{1}{2} s$ on one of the semi-infinite segments $L_{*}$ (Fig.4). The points $s \pm \pi$ fall into the points $\zeta^{ \pm}=(-1 / k z)^{ \pm} \in L^{ \pm}$, where $L^{+}\left(L^{-}\right)$is the left (right) bank of the contour $L$. Therefore

$$
f(s \pm \pi)=\chi_{3}^{ \pm}(\zeta)\left[M_{1}(s+\pi) \cosh \chi_{4}^{ \pm}(\zeta)+M_{2}(s+\pi) q^{1 / 2}(\zeta) \sinh \chi_{4}^{ \pm}(\zeta)\right],
$$

where $\zeta=-1 /(k z), \zeta \in L$. 
5.1.1 Functions $q^{1 / 2}(\zeta)$ and $\beta(s)$. We first establish a relation between the branches of the functions $q^{1 / 2}(\zeta)$ and $\beta(s)$. The single branch of the function $\beta(s)$ has been chosen by the condition $\beta(0)=\sin \eta$. As for the branch of the function $q^{1 / 2}(z)$, it meets the condition $q^{1 / 2}(0)=1$. By using relations $z=\tan \frac{1}{2} \eta \cot \frac{1}{2} s$ we observe that

$$
q^{1 / 2}(z)=\epsilon_{0} \frac{2 \beta(s)}{\sin \eta(1-\cos s)},
$$

where $\epsilon_{0}$ is real and $\left|\epsilon_{0}\right|=1$. To fix the sign of $\epsilon_{0}$ we put $z=0$. Because the point $s=\pi$ corresponds to the point $z=0$ on using formula (4.10), we find that $\beta(\pi)=-\sin \eta$, and the righthand side in (5.2) equals $-\epsilon_{0}$. So, $\epsilon_{0}=-1$. The desired relation between the functions $q^{1 / 2}(\zeta)$ and $\beta(s)$ is obtained by exploring formula (4.40)

$$
q^{1 / 2}(\zeta)=\frac{2 \beta(s)}{\sin \eta(1+\cos s)}
$$

5.1.2 The functions $\chi_{3}^{+}(\zeta)$ and $\chi_{3}^{-}(\zeta)$. At the next stage we derive a relation between the function $\chi_{3}(z), z \in L_{*}$, and the limiting values $\chi_{3}^{ \pm}(\zeta)$ on the banks $L^{ \pm}$of the contour $L$. Clearly, $\chi_{3}^{+}(\zeta)=-\chi_{3}^{-}(\zeta)$. By noticing that $k^{2} t_{1}^{2}=1 / t_{1}^{2}$, from (4.63) one can deduce

$$
\chi_{3}^{+}(\zeta)=\epsilon_{1} Q(z) \chi_{3}(z), \quad Q(z)=\frac{\beta(s) \sqrt{\cos ^{2} s-\sin ^{2} \theta}}{(\cos s-\sin \theta)(\cos s+\cos \eta)} \tan \frac{s}{2},
$$

where $\epsilon_{1}$ could be either 1 or -1 . We next fix the sign of the parameter $\epsilon_{1}$. The function $\sqrt{\cos ^{2} s-\sin ^{2} \theta}$ is single-valued in the strip $\Pi$ cut along the curves $\left(-\theta_{2},-\theta_{1}\right) \subset \Gamma_{0}$ and $\left(\theta_{1}, \theta_{2}\right) \subset \Gamma_{1}$. The branch of this function is fixed such that at the point $s=0$ it is equal to $\cos \theta$. Equivalently, $\sqrt{\cos ^{2} s-\sin ^{2} \theta} \sim \cos s$ as $\operatorname{Im} s \rightarrow \pm \infty$. Now, because the branch of the function $\chi_{3}(z)$ has been chosen by the condition $\chi_{3}(z) \sim z=\tan \frac{\eta}{2} \cot \frac{s}{2}$, as $z \rightarrow \infty(s \rightarrow 0)$, the sign of the parameter $\epsilon_{1}$ cannot be arbitrary and has to be defined. On setting $\zeta=0$ in the first equation in (5.4), we obtain

$$
\begin{gathered}
\chi_{3}^{+}(0)=-i t_{1} z_{1}=\tan ^{2} \frac{\eta}{2} \cot \left(\frac{\pi}{4}-\frac{\theta}{2}\right), \\
Q(z) \sim \tan \frac{\eta}{2} \cot \left(\frac{\pi}{4}-\frac{\theta}{2}\right) \tan \frac{s}{2}, \quad \chi_{3}(z) \sim \tan \frac{\eta}{2} \cot \frac{s}{2}, \quad z \rightarrow \infty \quad(s \rightarrow 0) .
\end{gathered}
$$

Comparing the limiting value of the function $Q(z) \chi_{3}(z)$ as $z \rightarrow \infty(s \rightarrow 0)$ with $\chi_{3}^{+}(0)$ yields $\epsilon_{1}=1$. Therefore

$$
\chi_{3}^{ \pm}(\zeta)= \pm Q(z) \chi_{3}(z)
$$

5.1.3 The functions $\chi_{4}^{+}(\zeta)$ and $\chi_{4}^{-}(\zeta)$. By the Sokhotski-Plemelj formulae applied to the function $\chi_{2}(z)$ in $(4.63)$ we obtain

$$
\chi_{2}^{ \pm}(\zeta)= \pm \frac{\log l_{1}(\zeta)+\pi i}{2 q^{1 / 2}(\zeta)}+\chi_{2}(\zeta)
$$


where $\chi_{2}(\zeta)$ is the principal value of the integral in (4.63). On using (4.40) we have $\log l_{1}(\zeta)=$ $\log l_{1}(z)$. Therefore, the limiting values of the function $\chi_{4}(z)=q^{1 / 2}(z) \chi_{2}(z)$ on the banks $L^{ \pm}$can be written as follows:

$$
\chi_{4}^{ \pm}(\zeta)= \pm \frac{\log l_{1}(z)+\pi i}{2}+\chi_{5}(\zeta)+\chi_{6}(\zeta)
$$

Here

$$
\begin{aligned}
& \chi_{5}(\zeta)=-\frac{\zeta q^{1 / 2}(\zeta)}{\pi i} \int_{0}^{t_{1}} \frac{\left[\log l_{1}(t)+\pi i\right] d t}{q^{1 / 2}(t)\left(t^{2}-\zeta^{2}\right)} \\
& \chi_{6}(\zeta)=-\zeta q^{1 / 2}(\zeta) \int_{1}^{z_{1}} \frac{d t}{q^{1 / 2}\left(t^{+}\right)\left(t^{2}-\zeta^{2}\right)}
\end{aligned}
$$

Notice that the integrand in the first integral in (5.9) is even and therefore

$$
\chi_{5}(\zeta)=\frac{q^{1 / 2}(\zeta)}{2 \pi i} \int_{L} \frac{\left[\log l_{1}(t)+\pi i\right] d t}{q^{1 / 2}(t)(t-\zeta)} .
$$

On making the substitutions $\zeta=-1 /(k z)$ and $t=-1 /(k \tau)$ and using formulae (4.40) and (5.3), we transform the above integral

$$
\chi_{5}(\zeta)=-\frac{q^{1 / 2}(z)}{2 \pi i} \int_{L_{*}} \frac{\left[\log l_{1}(\tau)+\pi i\right] d \tau}{q^{1 / 2}(\tau)(\tau-z)}-\frac{q^{1 / 2}(z)}{2 \pi i z} \int_{L_{*}} \frac{\left[\log l_{1}(t)+\pi i\right] d t}{q^{1 / 2}(t)} .
$$

Also, from (4.41) and (5.10)

$$
\chi_{5}(\zeta)=\chi_{5}(z)-\frac{q^{1 / 2}(z)}{2 \pi i} \int_{L \cup L_{*}} h(t) d t+\frac{d^{0}}{z} q^{1 / 2}(z),
$$

where

$$
h(t)=\frac{\log l_{1}(t)+\pi i}{q^{1 / 2}(t)(t-z)} .
$$

The function $h(t)$ is holomorphic in the domain $D$ (Fig.4) aside from a semi-circle of sufficiently small radius $\rho$ and centre $z \in L_{*}$. It is continuous up to the boundary apart from the points $z_{1}^{ \pm}, z_{2}^{ \pm} \in$ $\gamma_{1}^{ \pm}$, where the function $h(t)$ has logarithmic singularities. By the Cauchy theorem

$$
\left(\int_{-t_{R}}^{t_{-\rho}}+\int_{t_{\rho}}^{t_{R}}+\int_{c_{\rho}}+\int_{C_{R}}+\oint_{\mathbf{a}}\right) h(t) d t=0
$$

Because of (4.46), the last integral in (5.14) over the cross-section a can be transformed as follows:

$$
\begin{aligned}
\oint_{\mathbf{a}} h(t) d t & =\int_{1}^{1 / k} \frac{\log l_{1}^{+}(t)+\log l_{1}^{-}(t)+2 \pi i}{q^{1 / 2}\left(t^{+}\right)(t-z)} d t \\
& =-2 \pi i\left(\int_{1}^{z_{1}}+\int_{z_{2}}^{1 / k}\right) \frac{d t}{q^{1 / 2}\left(t^{+}\right)(t-z)} .
\end{aligned}
$$


On the circular arc $c_{\rho}$,

$$
h(t)=\frac{\log l_{1}(z)+\pi i}{q^{1 / 2}(z)} \frac{1}{t-z}+h_{1}(t), \quad\left|h_{1}(t)\right| \leqslant A_{1}, \quad A_{1}=\text { const. }
$$

On the contour $C_{R}$, the function $h(t)$ admits the representation $h(t)=h_{2}(t) t^{-3}$, where $\left|h_{2}(t)\right| \leqslant$ $A_{2}, A_{2}=$ const. Finally, taking $\rho \rightarrow 0$ and $R \rightarrow \infty$, from (5.14) and (5.12) we find

$$
\chi_{5}(\zeta)=\chi_{5}(z)-\frac{\log l_{1}(z)+\pi i}{2}-q^{1 / 2}(z)\left(\int_{1}^{z_{1}}+\int_{z_{2}}^{1 / k}\right) \frac{d t}{q^{1 / 2}\left(t^{+}\right)(t-z)}+\frac{d^{0}}{z} q^{1 / 2}(z) .
$$

Now we transform the integral $\chi_{6}(\zeta)$. On using formula (4.41), we find it is a straightforward matter to show that

$$
\chi_{6}(\zeta)=-\frac{d^{0}}{\zeta} q^{1 / 2}(\zeta)-\frac{q^{1 / 2}(\zeta)}{\zeta} \int_{1}^{z_{1}} \frac{t^{2} d t}{q^{1 / 2}\left(t^{+}\right)\left(t^{2}-\zeta^{2}\right)} .
$$

By making the substitutions $\zeta=-1 /(k z)$ and $t=-1 /(k \tau)$ the integral becomes

$$
\chi_{6}(\zeta)=-\frac{d^{0}}{z} q^{1 / 2}(z)+z q^{1 / 2}(z) \int_{z_{2}}^{1 / k} \frac{d t}{q^{1 / 2}\left(t^{+}\right)\left(t^{2}-z^{2}\right)} .
$$

Here we used that for $\tau_{1}=-\tau, q^{1 / 2}\left(\tau^{+}\right)=q^{1 / 2}\left(\tau_{1}^{-}\right)=-q^{1 / 2}\left(\tau_{1}^{+}\right)$.

Having transformed the integrals $\chi_{5}(\zeta)$ and $\chi_{6}(z)$ we obtain from (5.8), (5.17) and (5.19) the following relation for the limiting values $\chi_{4}^{ \pm}(\zeta)$ :

$$
\chi_{4}^{ \pm}(\zeta)=\chi_{4}(z) \pm \frac{\log l_{1}(z)+\pi i}{2}+\chi_{7}(z)
$$

where

$$
\begin{aligned}
\chi_{7}(z) & =-\frac{\log l_{1}(z)+\pi i}{2}-\frac{q^{1 / 2}(z)}{2}\left(\int_{1}^{z_{1}}+\int_{z_{2}}^{1 / k}\right)\left(\frac{1}{t-z}+\frac{1}{t+z}\right) \frac{d t}{q^{1 / 2}\left(t^{+}\right)} \\
& =-\frac{\log l_{1}(z)+\pi i}{2}-\frac{q^{1 / 2}(z)}{2}\left(\int_{-1 / k}^{-z_{2}}+\int_{-z_{1}}^{-1}+\int_{1}^{z_{1}}+\int_{z_{2}}^{1 / k}\right) \frac{d t}{q^{1 / 2}\left(t^{+}\right)(t-z)} .
\end{aligned}
$$

The function $\chi_{7}(z)$ is an even holomorphic function in the $z$-plane cut along the curves $\gamma_{0}, \gamma_{1}$. The first relation in (5.21) reveals that the function $\chi_{7}(z)$ is bounded at infinity. We next prove that $\chi_{7}(z)$ is identically equal to zero. Indeed, from the relation (4.46) and the Sokhotski-Plemelj formulae it follows that $\chi_{7}^{+}(t)+\chi_{7}^{-}(t)=0$ for $t \in \gamma_{1}$. Since the function $\chi_{7}(z)$ is even the same relation is valid on the curve $\gamma_{0}: \chi_{7}^{+}(t)+\chi_{7}^{-}(t)=0$. Thus, the function $\chi_{7}(z)$ is bounded at infinity and at the ends of the contours $\gamma_{0}, \gamma_{1}$, it is holomorphic in $\mathbb{C} \backslash\left\{\gamma_{0} \cup \gamma_{1}\right\}$, continuous up to the boundary $\gamma_{0} \cup \gamma_{1}$ and it satisfies the boundary condition

$$
\chi_{7}^{+}(t)=-\chi_{7}^{-}(t), \quad t \in \gamma_{0} \cup \gamma_{1} .
$$

This Riemann-Hilbert problem has the trivial solution only. So, $\chi_{7}(z) \equiv 0, z \in \mathbb{C}$. 
Since formula (5.1) requires the values of the functions $\cosh \chi_{4}^{ \pm}(\zeta)$ and $\sinh \chi_{4}^{ \pm}(\zeta)$ we find first

$$
e^{\chi_{4}^{+}(\zeta)}=i p(s) e^{\chi_{4}(z)}, \quad e^{\chi_{4}^{-}(\zeta)}=-\frac{i}{p(s)} e^{\chi_{4}(z)},
$$

where $p(s)=\exp \left\{\frac{1}{2} \log \lambda_{1}(s)\right\}$. Next,

$$
\begin{gathered}
\cosh \chi_{4}^{ \pm}(\zeta)=p_{1}(s) \cosh \chi_{4}(z) \pm p_{2}(s) \sinh \chi_{4}(z), \\
\sinh \chi_{4}^{ \pm}(\zeta)= \pm p_{2}(s) \cosh \chi_{4}(z)+p_{1}(s) \sinh \chi_{4}(z), \quad z \in L_{*},
\end{gathered}
$$

where

$$
\begin{aligned}
& p_{1}(s)=\frac{i}{2}\left(p(s)-\frac{1}{p(s)}\right)=\frac{\sqrt{3} \beta(s)}{2 \sqrt{\cos ^{2} s-\sin ^{2} \theta}} \\
& p_{2}(s)=\frac{i}{2}\left(p(s)+\frac{1}{p(s)}\right)=-\frac{i \cos s}{2 \sqrt{\cos ^{2} s-\sin ^{2} \theta}} .
\end{aligned}
$$

Here the branch of the function $\sqrt{\cos ^{2} s-\sin ^{2} \theta}$ is chosen in the same manner as in (5.4).

5.1.4 Relation for the functions $M_{1}(s)$ and $M_{2}(s)$. On substituting the expressions for $f(s)$ and $f(s \pm \pi)$ into equation (3.7) and using (4.66), (5.1), (5.3), (5.6) and (5.24) we obtain

$$
\begin{array}{r}
-i \tan \frac{s}{2}\left[\frac{2(\cos s-\cos \eta)}{\sin \eta(\cos s+1)} M_{2}(s+\pi) \cosh \chi_{4}(z)+\frac{\beta(s)}{\cos s+\cos \eta} M_{1}(s+\pi) \sinh \chi_{4}(z)\right] \\
=M_{1}(s) \cosh \chi_{4}(z)+\frac{2 \beta(s)}{\sin \eta(\cos s-1)} M_{2}(s) \sinh \chi_{4}(z) .
\end{array}
$$

The functions $\cosh \chi_{4}(z)$ and $\sinh \chi_{4}(z)$ are linearly independent over the field of meromorphic functions. Therefore, the conditions for the function $f(s)$ to satisfy equation (3.7) become

$$
\begin{gathered}
M_{1}(s)=-2 i \frac{\cos s-\cos \eta}{\sin \eta(\cos s+1)} \tan \frac{s}{2} M_{2}(s+\pi), \\
-i \tan \frac{s}{2} \frac{M_{1}(s+\pi)}{\cos s+\cos \eta}=\frac{2 M_{2}(s)}{\sin \eta(\cos s-1)}, \quad \operatorname{Re} s=0 .
\end{gathered}
$$

By the uniqueness theorem these conditions are valid not only on the imaginary axis but also in the whole $s$-plane. We next notice that the first equation in (5.27) coincides with the second one if we replace $s$ by $s+\pi$. This means that in order that the general solution of equation (3.10) is the general solution of the equation (3.7) it is necessary and sufficient that

$$
M_{2}(s)=-\frac{i \sin \eta \sin s(\cos s-1)}{2(\cos s+1)(\cos s+\cos \eta)} M_{1}(s+\pi) .
$$

Thus, the general solution of (3.7) depends upon the function $M_{1}(s)$ only and has the form

$$
f(s)=\chi_{3}(z)\left[M_{1}(s) \cosh \chi_{4}(z)-\frac{i \sin s \beta(s) M_{1}(s+\pi) \sinh \chi_{4}(z)}{(\cos s+1)(\cos s+\cos \eta)}\right], \quad-\pi \leqslant \operatorname{Re} s \leqslant \pi .
$$

We recall that $M_{1}(s)$ is an arbitrary $2 \pi$-periodic meromorphic function to be defined. We emphasize that the solution (5.29) is odd if and only if the function $M_{1}(s)$ is even. 


\subsection{Properties of the functions $\chi_{3}(z), \cosh \chi_{4}(z), \sinh \chi_{4}(z)$}

To identify the function $M_{1}(s)$ we need to study the properties of the functions

$$
f_{1}(s)=\chi_{3}(z) \cosh \chi_{4}(z), \quad f_{2}(s)=\frac{i \sin s \beta(s) \chi_{3}(z) \sinh \chi_{4}(z)}{(\cos s+1)(\cos s+\cos \eta)} .
$$

In the strip $-\pi \leqslant \operatorname{Re} s \leqslant \pi$, the functions $f_{1}(s)$ and $f_{2}(s)$ are odd, and it is sufficient to study their properties in the strip $0 \leqslant \operatorname{Re} s \leqslant \pi$. Analysis of the functions (4.63) and (5.30) indicates that in the strip $0 \leqslant \operatorname{Re} s \leqslant \pi$ the functions $f_{1}(s)$ and $f_{2}(s)$ may have singularities and zeros at the following points: $s=x \pm i \infty(0 \leqslant x \leqslant \pi), s=0, s=\eta, s=\theta_{1}^{ \pm} \in \Gamma_{1}^{ \pm}$(the corresponding points in the $z$-plane are $z=\mp t_{1}, z=\infty, z=1, z=z_{1}^{ \pm} \in \gamma_{1}^{ \pm}$) and also, because of the denominator of the function $f_{2}(s)$, at the points $s=\pi$ and $s=\pi-\eta$. Apart from the above points, the functions $f_{1}(s)$ and $f_{2}(s)$ are bounded and do not equal zero.

Now we look into the behaviour of the functions $f_{1}(s)$ and $f_{2}(s)$ as $\operatorname{Im} s \rightarrow-\infty, s \in \bar{\Pi}$. In the vicinity of the end point $t=t_{1}$ of the contour $L$,

$$
\begin{gathered}
\chi_{3}(z)=A_{0}\left(z-t_{1}\right)^{1 / 2}+O\left\{\left(z-t_{1}\right)^{3 / 2}\right\} \\
\chi_{4}(z)=\frac{1}{6} \log \left(z-t_{1}\right)+O(1), \quad z \rightarrow t_{1}, \quad A_{0}=\mathrm{const} \neq 0 .
\end{gathered}
$$

Therefore,

$$
\begin{gathered}
\chi_{3}(z) \cosh \chi_{4}(z)=A_{1}\left(z-t_{1}\right)^{1 / 3}+A_{2}\left(z-t_{1}\right)^{2 / 3}+O\left\{\left(z-t_{1}\right)^{4 / 3}\right\}, \\
\chi_{3}(z) \sinh \chi_{4}(z)=-A_{1}\left(z-t_{1}\right)^{1 / 3}+A_{2}\left(z-t_{1}\right)^{2 / 3}+O\left\{\left(z-t_{1}\right)^{4 / 3}\right\}, \quad z \rightarrow t_{1},
\end{gathered}
$$

where $A_{1}, A_{2}$ are non-zero constants. Next, since

$$
z-t_{1} \sim 2 t_{1} e^{-i s}, \quad \frac{i \sin s \beta(s)}{(\cos s+1)(\cos s+\cos \eta)} \sim 1, \quad \operatorname{Im} s \rightarrow-\infty, \quad s \in \bar{\Pi},
$$

it follows that

$$
\begin{gathered}
f_{1}(s)=A_{1}^{\prime} e^{-i s / 3}+A_{2}^{\prime} e^{-2 i s / 3}+O\left(e^{-4 i s / 3}\right), \\
f_{2}(s)=-A_{1}^{\prime} e^{-i s / 3}+A_{2}^{\prime} e^{-2 i s / 3}+O\left(e^{-4 i s / 3}\right), \quad \operatorname{Im} s \rightarrow-\infty, \quad s \in \bar{\Pi},
\end{gathered}
$$

where $A_{1}^{\prime}, A_{2}^{\prime}$ are some non-zero constants. So, the functions $f_{1}(s)$ and $f_{2}(s)$ decay exponentially at the lower end of the strip. Since they are odd functions, they decay exponentially at the upper end as well:

$$
\begin{gathered}
f_{1}(s)=-A_{1}^{\prime} e^{i s / 3}-A_{2}^{\prime} e^{2 i s / 3}+O\left(e^{4 i s / 3}\right), \\
f_{2}(s)=A_{1}^{\prime} e^{i s / 3}-A_{2}^{\prime} e^{2 i s / 3}+O\left(e^{4 i s / 3}\right), \quad \operatorname{Im} s \rightarrow+\infty, \quad s \in \bar{\Pi} .
\end{gathered}
$$

Next, we analyse these functions at the points $s=0$ and $s=\pi$. As $z \rightarrow \infty, \chi_{3}(z) \sim z$, and the function $\chi_{4}(z)$ is bounded and continuous at the point $z=\infty$. Since this function is odd then, clearly, $\chi_{4}(\infty)=0$. This means $\chi_{4}(z) \sim A_{3} z^{-1}, z \rightarrow \infty, A_{3}=$ const $\neq 0$. Then

$$
\cosh \chi_{4}(z)=1+O\left(z^{-2}\right), \quad \sinh \chi_{4}(z)=A_{3} z^{-1}+O\left(z^{-3}\right), \quad z \rightarrow \infty .
$$

From the above formulae it follows that the point $s=0$ is a simple pole of the function $f_{1}(s)$ and a 
simple zero of the function $f_{2}(s)$. To recover the properties of these functions at the point $s=\pi$, we notice that

$$
\cosh \chi_{4}^{+}(0)=p_{1}(0) \neq 0, \quad \sinh \chi_{4}^{+}(0)=p_{2}(0) \neq 0 .
$$

The function $f_{1}(s)$ is clearly bounded and non-zero at the point $s=\pi$. As for the second function, $f_{2}(s)$, because of the factor $\sin s /(\cos s+1)$, it has a simple pole at the point $s=\pi$.

Now we prove that both functions are bounded and non-zero at the point $s=\eta$. The image of this point in the $z$-plane is the point $z=1$. Analysis of the Cauchy integral in (4.63) shows (Gakhov (9))

$$
\chi_{2}(z)=-\frac{\pi i}{2 q_{*}(1)}(z-1)^{-1 / 2}+\chi_{*}(z)
$$

where $q_{*}(z)=(z-1)^{-1 / 2} q^{1 / 2}(z)$ and $\chi_{*}(z)$ is a function bounded at $z=1$ and $\chi_{*}(1) \neq 0$. Then by the definition (4.67)

$$
\chi_{4}(z)=-\frac{\pi i}{2}+q_{*}(1) \chi_{*}(z)(z-1)^{1 / 2}, \quad z \rightarrow 1 .
$$

Hence $\cosh \chi_{4}(z) \sim-i A_{4}(z-1)^{1 / 2}, \sinh \chi_{4}(z) \sim-i, z \rightarrow 1$, where $A_{4}=q_{*}(1) \chi_{*}(1) \neq 0$. Use of this result and also of the asymptotics

$$
\begin{aligned}
\chi_{3}(z) & \sim A_{5}(z-1)^{-1 / 2}, \quad z \rightarrow 1, \quad A_{5}=\text { const } \neq 0, \\
\beta(s) & \sim A_{6}(s-\eta)^{1 / 2}, \quad s \rightarrow \eta, \quad A_{6}=\text { const } \neq 0,
\end{aligned}
$$

yields the boundness of the functions $f_{1}(s), f_{2}(s)$ at the point $s=\eta$ and $f_{j}(\eta) \neq 0, j=1,2$.

The next potential singular point is $s=\pi-\eta$. Its image is the point $z=1 / k$. From (4.63) we find $\chi_{4}(z) \sim A_{7}(z-1 / k)^{1 / 2}, z \rightarrow 1 / k, A_{7}=$ const $\neq 0$. Therefore, $\cosh \chi_{4}(1 / k)=1$ and the function $\sinh \chi_{4}(z)$ has a zero of order $\frac{1}{2}$. Because the function $\beta(s)$ has also a zero of order $\frac{1}{2}$ at the point $s=\pi-\eta$, both functions $f_{1}(s)$ and $f_{2}(s)$ are bounded and do not equal zero at this point.

Finally, we analyse the behaviour of the functions $f_{1}(s), f_{2}(s)$ at the points $s=\theta_{1}^{ \pm} \in \Gamma_{1}^{ \pm}$. Since

$$
\begin{gathered}
\chi_{3}(z) \sim A_{8}\left(z-z_{1}\right)^{1 / 2}, \quad z \rightarrow z_{1}, \quad A_{8}=\text { const } \neq 0, \\
\chi_{4}(z) \sim \mp \frac{1}{2} \log \left(z-z_{1}\right), \quad z \rightarrow z_{1}^{ \pm}, \quad z \notin \gamma_{1},
\end{gathered}
$$

it follows that in the vicinity of the points $s=\theta_{1}^{ \pm} \in \Gamma_{1}^{ \pm}$,

$$
\begin{gathered}
\cosh \chi_{4}(z) \sim A_{9}\left(s-\theta_{1}\right)^{-1 / 2}, \quad \sinh \chi_{4}(z) \sim \pm A_{9}\left(s-\theta_{1}\right)^{-1 / 2}, \\
\chi_{3}(z) \sim A_{8}^{\prime}\left(s-\theta_{1}\right)^{1 / 2}, \quad s \rightarrow \theta_{1}^{ \pm}, \quad s \notin \Gamma_{1},
\end{gathered}
$$

where $A_{8}^{\prime}, A_{9}$ are non-zero constants. On using that $\beta^{+}\left(\theta_{1}\right)=-\beta^{-}\left(\theta_{1}\right) \neq 0$, we obtain that the functions $f_{1}(s)$ and $f_{2}(s)$ are bounded at the point $s=\theta_{1}$ and, in addition, $f_{j}^{+}\left(\theta_{1}\right)=f_{j}^{-}\left(\theta_{1}\right) \neq 0$, $j=1,2$.

In summary, the odd functions $f_{1}(s)$ and $f_{2}(s)$ are bounded and non-zero everywhere in a finite part of the strip $-\pi \leqslant \operatorname{Re} s \leqslant \pi$ apart from the points $s=0$ and $s= \pm \pi$. At the point $s=0$, the function $f_{1}(s)$ has a simple pole, and the function $f_{2}(s)$ has a simple zero. At the points $s= \pm \pi$, the function $f_{1}(s)$ is bounded and non-zero, and the function $f_{2}(s)$ has simple poles. At the ends of the strip these functions decay exponentially as described in (5.34), (5.35). 


\subsection{Definition of the function $M_{1}(s)$}

First we prove that the even $2 \pi$-periodic function $M_{1}(s)$ is entire. Equation (3.7) and formulae (4.15) indicate that the function $M_{1}(s)$ may have simple poles at the points $\pm(\pi / 2+\theta)+2 \pi n$, $n \in \mathbb{Z}$, and at the zeros of the function $\Delta(s)=i \sqrt{3} \beta(s) \cos s$, that is, at the points $\pi / 2+\pi n$, $\pm \eta+\pi n, n \in \mathbb{Z}$. Analyse the function

$$
f(s)=f_{1}(s) M_{1}(s)-f_{2}(s) M_{1}(s+\pi)
$$

at the point $s=\pi / 2+\theta$. Since $s=3 \pi / 2+\theta$ is not a pole of the function $M_{1}(s)$ and $f_{1}(\pi / 2+\theta) \neq 0$ then from the analyticity of the function $f(s)$ at the point $s=\pi / 2+\theta$, it follows the analyticity of the function $M_{1}(s)$ at this point and therefore, by the periodicity, at the points $s= \pm(\pi / 2+\theta)+2 \pi n$, $n \in \mathbb{Z}$.

It has been shown that the functions $f_{1}(s)$ and $f_{2}(s)$ are non-zero at the points $s= \pm \eta$. Clearly, the function $f(s)$ is analytic at these points if the function $M_{1}(s)$ is analytic at the points $\pm \eta+\pi n$, $n \in \mathbb{Z}$.

Now we study the function $M_{1}(s)$ at the point $s=\pi / 2$. Represent it as follows: $M_{1}(s)=$ $M_{0}(s) \sec s$, where the function $M_{0}(s)$ is an even $2 \pi$-periodic function. From (5.43) and (5.30) the residue of the function $f(s)$ at the point $s=\pi / 2$ becomes

$$
\operatorname{res}_{s=\pi / 2} f(s)=-f_{1}\left(\frac{\pi}{2}\right) M_{0}\left(\frac{\pi}{2}\right)-f_{2}\left(\frac{\pi}{2}\right) M_{0}\left(\frac{3 \pi}{2}\right)=-M_{0}\left(\frac{\pi}{2}\right) \chi_{3}^{+}\left(z_{0}\right) e^{\chi_{4}^{+}\left(z_{0}\right)},
$$

where $z_{0}=\tan \frac{1}{2} \eta=1 / \sqrt{k}$. Here we used the analyticity of the functions $f_{j}(s), j=1,2$, in the strip $\Pi$, their definition (5.30) and also the relations $M_{0}(3 \pi / 2)=M_{0}(-\pi / 2)=M_{0}(\pi / 2)$ (the function $M_{0}(s)$ is even and $2 \pi$-periodic). We can further reason, on using the analyticity of the function $f(s)$ at the point $s=\pi / 2$ and also $\chi_{3}^{+}\left(z_{0}\right) \neq 0$, that $M_{0}(\pi / 2)=0$. This means that the function $M_{1}(s)$ is analytic at the points $\pi / 2+\pi n, n \in \mathbb{Z}$.

At the point $s=0$, the function $f_{1}(s)$ has a simple pole. For the function $f(s)$ to be analytic at this point, it is necessary that $M_{1}(0)=0$. But the function $M_{1}(s)$ is even and therefore its zero at the point $s=0$ is of order not less than two. Note that although the function $f_{2}(s)$ has simple poles at the points $s= \pm \pi$, the function $f(s)$ is bounded at these points.

On using (3.8), (5.34), (5.35) and (5.43), we find that the function $M_{1}(s)$ may grow exponentially as $\operatorname{Im} s \rightarrow \pm \infty, s \in \bar{\Pi}$ as follows:

$$
\left|M_{1}(s)\right| \leqslant A_{*} e^{\frac{7}{3}|\operatorname{Im} s|}, \quad A_{*}=\text { const. }
$$

Thus, the most general form of the even $2 \pi$-periodic entire function $M_{1}(s)$ that meets all the above conditions is

$$
M_{1}(s)=(1-\cos s)\left(B_{0}+B_{1} \cos s\right),
$$

where $B_{0}, B_{1}$ are arbitrary constants. Then the desired odd solution of equation (3.7) in the class (3.8) in the strip $\bar{\Pi}$ has the form

$$
\begin{aligned}
f(s)= & \chi_{3}(z)\left\{(1-\cos s)\left(B_{0}+B_{1} \cos s\right) \cosh \left[q^{1 / 2}(z) \chi_{2}(z)\right]\right. \\
& \left.-\frac{i \sin s \beta(s)}{\cos s+\cos \eta}\left(B_{0}-B_{1} \cos s\right) \sinh \left[q^{1 / 2}(z) \chi_{2}(z)\right]\right\}, \quad-\pi \leqslant \operatorname{Re} s \leqslant \pi .
\end{aligned}
$$

Outside the strip $\bar{\Pi}$, the solution can be found by analytical continuation by means of equation (3.7). 


\section{Analysis of the spectral functions $\mathcal{S}_{1}(s), \mathcal{S}_{2}(s)$}

\subsection{Asymptotics at infinity}

First we write down the asymptotics at infinity of the functions $f(s)$ and $\psi(s)$. From (5.34), (5.35), (5.43) and (5.46) for the odd function $f(s)$ we obtain

$$
f(s)=\mp A e^{\mp \frac{5}{3} i s}+O\left(e^{\mp \frac{2}{3} i s}\right), \quad \operatorname{Im} s \rightarrow \pm \infty, \quad A=-\frac{1}{2} A_{2}^{\prime} B_{1} .
$$

To analyse the asymptotics of the function $\psi(s)$ as $\operatorname{Im} s \rightarrow-\infty$, we make the substitutions $x=$ $e^{-i s}, x_{0}=e^{-i s_{0}}, \xi=e^{-i \tau}$ in (3.12) $\left(C_{2}=0\right)$. Then the kernel (3.14) becomes

$$
\Lambda(\tau, s)=-\frac{4 i \xi^{2}\left(x-x_{0}\right)}{(\xi-x)\left(\xi-x_{0}\right)\left(\xi^{2}+1\right)} .
$$

We next introduce the function

$$
\psi_{*}(s)=\frac{\cos s}{4 \pi i} \int_{-i \infty}^{i \infty} \Lambda(\tau, s) f(\tau) d \tau=\frac{\left(x-x_{0}\right)\left(x^{2}+1\right)}{2 \pi i x} \int_{0}^{\infty} \frac{\xi f_{0}(\xi) d \xi}{(\xi-x)\left(\xi-x_{0}\right)\left(\xi^{2}+1\right)},
$$

where $f_{0}(\xi)=f(i \log \xi)$. From (6.1) we may conclude that the function $\xi f_{0}(\xi)$ has a power singularity at the point $\xi=0$ :

$$
\xi f_{0}(\xi)=A \xi^{-2 / 3}+O\left(\xi^{1 / 3}\right), \quad \xi \rightarrow 0 .
$$

Analysis of the Cauchy integral (6.3) at the point $x=0$ gives

$$
\psi_{*}(s)=\frac{A}{\sqrt{3}} e^{\pi i / 6+\frac{5}{3} i s}+\psi_{0}(s) e^{i s}, \quad \operatorname{Im} s \rightarrow-\infty,
$$

where $\psi_{0}(s)$ is a bounded function with a definite finite limit as $\operatorname{Im} s \rightarrow-\infty$. Then

$$
\psi(s)=\frac{i A}{\sqrt{3}} e^{\frac{5}{3} i s}+\psi_{1}(s) e^{i s}, \quad \operatorname{Im} s \rightarrow-\infty,
$$

where $\psi_{1}(s)=\frac{1}{2} C_{1}-\psi_{0}(s-\pi)$. We notice that the function $\psi(s)$ is even and therefore

$$
\psi(s)=\frac{i A}{\sqrt{3}} e^{-\frac{5}{3} i s}+\psi_{1}(-s) e^{-i s}, \quad \operatorname{Im} s \rightarrow+\infty .
$$

Having found the asymptotics at infinity of the functions $\psi(s)$ and $f(s)$ we now define the asymptotics of the spectral functions. Formulae (3.17), (3.18), (6.1), (6.6) and (6.7) imply

$$
\mathcal{S}_{1}(s)=A_{ \pm} e^{ \pm i s / 3}+S_{ \pm}(s) e^{ \pm i s}, \quad \operatorname{Im} s \rightarrow \pm \infty,
$$

where

$$
A_{+}=\frac{4}{\sqrt{6}}(i-1) A \cos 2 \varphi_{0}, \quad A_{-}=-i A_{+},
$$

and the bounded functions $S_{ \pm}(s)$ have the definite finite limits $S_{+}(i \infty)=-S_{-}(-i \infty)$. Thus, the 
spectral function $\mathcal{S}_{1}(s)$ decays exponentially as $\operatorname{Im} s \rightarrow \pm \infty: \mathcal{S}_{1}(s)=O\left(e^{-\frac{1}{3} \operatorname{Im} s \mid}\right)$. To specify the asymptotics at infinity of the second spectral function, we notice that

$$
\begin{aligned}
\mathcal{S}_{1}(s+\pi)+\mathcal{S}_{1}(s-2 \pi)= & A_{ \pm}\left(e^{ \pm i \pi / 3}+e^{\mp 2 \pi / 3}\right) e^{ \pm i s / 3} \\
& +\left[S_{ \pm}(s+\pi)+S_{ \pm}(s-2 \pi)\right] e^{ \pm i s}, \quad \operatorname{Im} s \rightarrow \pm \infty .
\end{aligned}
$$

Since the first term in the right-hand side in (6.10) vanishes, on using formula (3.2), we obtain that the spectral function $\mathcal{S}_{2}(s)$ is bounded as $\operatorname{Im} s \rightarrow \pm \infty$ and it has the definite finite limits $\mathcal{S}_{2}( \pm i \infty)$ such that $\mathcal{S}_{2}(i \infty)=-\mathcal{S}_{2}(-i \infty)$.

\subsection{Definition of the arbitrary constants}

In general, the spectral functions may have some inadmissible poles which should be eliminated by fixing the arbitrary constants. The function $\mathcal{S}_{1}(s)$ must be analytic everywhere in the strip $-3 \pi / 4 \leqslant$ $\operatorname{Re} s \leqslant 3 \pi / 4$ apart from the point $s=\varphi_{0}$, where it has to have a simple pole to reproduce the incident field (2.8)

$$
\underset{s=\varphi_{0}}{\operatorname{res}} \mathcal{S}_{1}(s)=1
$$

The function $\mathcal{S}_{2}(s)$ must be free of poles everywhere in the strip $-\pi / 4 \leqslant \operatorname{Re} s \leqslant \pi / 4$. Among the poles of the function $\Sigma(s), s=\varphi_{0}+\pi n, s=-\varphi_{0}+\pi / 2+\pi n(n=0, \pm 1, \pm 2, \ldots)$ determine now those which violate the analyticity of the spectral functions $\mathcal{S}_{1}(s)$ and $\mathcal{S}_{2}(s)$ in the strips $\{-3 \pi / 4 \leqslant$ $\operatorname{Re} s \leqslant 3 \pi / 4\} \backslash\left\{s=\varphi_{0}\right\}$ and $\{-\pi / 4 \leqslant \operatorname{Re} s \leqslant \pi / 4\}$, respectively.

Let first $\pi / 4 \leqslant \varphi_{0} \leqslant 3 \pi / 4$. Then there are only two inadmissible poles at the points $s=\varphi_{0}-\pi$ and $s=-\varphi_{0}+\pi / 2$ for the function $\mathcal{S}_{1}(s)$. As for the function $\mathcal{S}_{2}(s)$, it has only one forbidden pole at the point $s=\pi / 2-\varphi_{0}$. In the next zone, $-\pi / 4 \leqslant \varphi_{0} \leqslant \pi / 4$, the poles, we need to remove, are $s=-\varphi_{0}-\pi / 2$ and $s=-\varphi_{0}+\pi / 2$ for the function $\mathcal{S}_{1}(s)$ and $s=\varphi_{0}$ for the second function $\mathcal{S}_{2}(s)$. In the last possible case, $-3 \pi / 4 \leqslant \varphi_{0} \leqslant-\pi / 4$, the function $\mathcal{S}_{1}(s)$ has two poles $s=-\varphi_{0}-\pi / 2$ and $s=\varphi_{0}+\pi$, and the function $\mathcal{S}_{2}(s)$ has again only one pole $s=-\varphi_{0}-\pi / 2$ which should be removed. Thus, we have found three new additional conditions

$$
\underset{s=\alpha_{1}}{\operatorname{res}} \mathcal{S}_{1}(s)=0, \quad \underset{s=\alpha_{2}}{\text { res }} \mathcal{S}_{1}(s)=0, \quad \underset{s=\alpha_{3}}{\operatorname{res}} \mathcal{S}_{2}(s)=0
$$

where

$$
\begin{gathered}
\alpha_{1}=\left\{\begin{array}{ll}
\varphi_{0}-\pi, & \pi / 4 \leqslant \varphi_{0} \leqslant 3 \pi / 4, \\
-\varphi_{0}-\pi / 2, & -3 \pi / 4 \leqslant \varphi_{0} \leqslant \pi / 4,
\end{array} \alpha_{2}= \begin{cases}-\varphi_{0}+\pi / 2, & -\pi / 4 \leqslant \varphi_{0} \leqslant 3 \pi / 4 \\
\varphi_{0}+\pi, & -3 \pi / 4 \leqslant \varphi_{0} \leqslant-\pi / 4\end{cases} \right. \\
\alpha_{3}= \begin{cases}-\varphi_{0}+\pi / 2, & \pi / 4 \leqslant \varphi_{0} \leqslant 3 \pi / 4 \\
\varphi_{0}, & -\pi / 4 \leqslant \varphi_{0} \leqslant \pi / 4 \\
-\varphi_{0}-\pi / 2, & -3 \pi / 4 \leqslant \varphi_{0} \leqslant-\pi / 4 .\end{cases}
\end{gathered}
$$

To fix the four constants $B_{0}, B_{1}, C_{0}$ and $C_{1}$, we have the four conditions (6.11) and (6.12). On evaluating the residues in (6.11) and (6.12), we can express these conditions in terms of the functions $f(s)$ and $\psi(s)$. Let

$$
\delta_{ \pm}=\sin \theta \pm \sin \left(\varphi_{0}+\pi / 4\right)
$$


Suppose first that $-3 \pi / 4 \leqslant \varphi_{0} \leqslant-\pi / 4$. Then by using (6.13) and substituting the expressions (3.17) and (3.18) into the conditions (6.11), (6.12) we replace them by

$$
\begin{gathered}
\delta_{+}\left[f\left(\varphi_{0}-\pi / 4\right)-1\right]=0, \quad f\left(\varphi_{0}+3 \pi / 4\right)=0, \\
\psi\left(\varphi_{0}-\pi / 4\right)=0, \quad \psi\left(\varphi_{0}+3 \pi / 4\right)=1 .
\end{gathered}
$$

It will be shown in section 8.1 that the assumption $\delta_{+}=0$ implies the existence of non-physical reflected wave in the shadow domain. The parameter $\delta_{+}$vanishes if and only if $\varphi_{0}=-\pi / 4-\theta$ and $\theta$ is real. Therefore we exclude this case and from the first condition in equations (6.15) we have $f\left(\varphi_{0}-\pi / 4\right)=1$.

Correspondingly, in the case $-\pi / 4 \leqslant \varphi_{0} \leqslant \pi / 4$, the conditions (6.11), (6.12) become

$$
\begin{gathered}
f\left(\varphi_{0}-\pi / 4\right)=1, \quad \delta_{-} f\left(\varphi_{0}+3 \pi / 4\right)=0, \\
\psi\left(\varphi_{0}-\pi / 4\right)=0, \quad \psi\left(\varphi_{0}+3 \pi / 4\right)=1 .
\end{gathered}
$$

Analysis of the reflected waves and the surface waves (section 8.1) indicates that the case $\varphi_{0}=$ $\theta-\pi / 4, \theta$ is real $\left(\delta_{-}=0\right)$, is singular and gives rise to the infinite reflected and surface coefficients. Therefore $\delta_{-} \neq 0$ and $f\left(\varphi_{0}+3 \pi / 4\right)=0$. Finally, if $\pi / 4 \leqslant \varphi_{0} \leqslant 3 \pi / 4$, then the additional conditions can be written in the form

$$
\begin{gathered}
f\left(\varphi_{0}-\pi / 4\right)=1, \quad \delta_{-} f\left(-\varphi_{0}+5 \pi / 4\right)=\sin \left(\varphi_{0}+\pi / 4\right), \\
\psi\left(\varphi_{0}-\pi / 4\right)=0, \quad \psi\left(-\varphi_{0}+5 \pi / 4\right)=0 .
\end{gathered}
$$

The assumption $\delta_{-}=0$ means $\varphi_{0}=3 \pi / 4-\theta$ and $\theta$ is real, $\theta \neq 0$. It conflicts with the requirement for the function $\mathcal{S}_{2}(s)$ to be analytic everywhere in the strip $3 \pi / 4 \leqslant \operatorname{Re} s \leqslant 5 \pi / 4$. Also, if $\delta_{-}=0$, then the reflected and the surface coefficients become infinite (section 8.1). Therefore, $\delta_{-} \neq 0$ and

$$
f\left(-\varphi_{0}+5 \pi / 4\right)=\delta_{*}, \quad \delta_{*}=\delta_{-}^{-1} \sin \left(\varphi_{0}+\pi / 4\right) .
$$

We next aim to find the constants $B_{j}, C_{j}(j=0,1)$ explicitly. Rewrite the expressions for the functions $f(s)$ and $\psi(s)$ as follows:

$$
\begin{aligned}
& f(s)=B_{0} \mathcal{F}_{0}(s)+B_{1} \mathcal{F}_{1}(s), \quad-\pi<\operatorname{Re} s<\pi, \\
& \psi(s)=C_{0}+C_{1} \cos s+B_{0} \mathcal{G}_{0}(s)+B_{1} \mathcal{G}_{1}(s), \quad-\pi<\operatorname{Re} s<\pi,
\end{aligned}
$$

where the functions $\mathcal{F}_{j}(s)$ and $\mathcal{G}_{j}(s)(j=0,1)$ are independent of the constants $B_{j}, C_{j}$ :

$$
\begin{gathered}
\mathcal{F}_{j}(s)=\cos ^{j} s \chi_{3}(z)\left\{(1-\cos s) \cosh \left[q^{1 / 2}(z) \chi_{2}(z)\right]-\frac{(-1)^{j} i \sin s \beta(s)}{\cos s+\cos \eta} \sinh \left[q^{1 / 2}(z) \chi_{2}(z)\right]\right\}, \\
\mathcal{G}_{j}(s)=\frac{\cos s}{4 \pi i} \int_{\Omega} \Lambda(\tau, s) \mathcal{F}_{j}(\tau-\pi) d \tau, \quad j=0,1
\end{gathered}
$$

Now it is straightforward to write down the desired constants in explicit form. By substituting the expressions (6.19) into equations (6.15) we obtain

$$
B_{0}=\frac{\mathcal{F}_{1}^{+}-\delta \mathcal{F}_{1}^{-}}{\mathcal{D}}, \quad B_{1}=-\frac{\mathcal{F}_{0}^{+}-\delta \mathcal{F}_{0}^{-}}{\mathcal{D}}
$$




$$
\begin{gathered}
C_{0}=-\frac{1}{2}\left[B_{0}\left(\mathcal{G}_{0}^{-}+\mathcal{G}_{0}^{+}\right)+B_{1}\left(\mathcal{G}_{1}^{-}+\mathcal{G}_{1}^{+}\right)-1+\frac{\delta}{\delta_{*}}\right] \\
C_{1}=-\frac{1}{2 \cos \left(\varphi_{0}-\pi / 4\right)}\left[B_{0}\left(\mathcal{G}_{0}^{-}-\mathcal{G}_{0}^{+}\right)+B_{1}\left(\mathcal{G}_{1}^{-}-\mathcal{G}_{1}^{+}\right)+1-\frac{\delta}{\delta_{*}}\right] .
\end{gathered}
$$

Here

$$
\begin{gathered}
\mathcal{F}_{j}^{-}=\mathcal{F}_{j}\left(\varphi_{0}-\pi / 4\right), \quad \mathcal{G}_{j}^{-}=\mathcal{G}_{j}\left(\varphi_{0}-\pi / 4\right), \quad \mathcal{D}=\mathcal{F}_{0}^{-} \mathcal{F}_{1}^{+}-\mathcal{F}_{0}^{+} \mathcal{F}_{1}^{-}, \\
\mathcal{F}_{j}^{+}= \begin{cases}\mathcal{F}_{j}\left(\varphi_{0}+3 \pi / 4\right), \quad-3 \pi / 4 \leqslant \varphi_{0} \leqslant \pi / 4, \\
\mathcal{F}_{j}\left(-\varphi_{0}+5 \pi / 4\right), \pi / 4 \leqslant \varphi_{0} \leqslant 3 \pi / 4,\end{cases} \\
\mathcal{G}_{j}^{+}= \begin{cases}\mathcal{G}_{j}\left(\varphi_{0}+3 \pi / 4\right), & -3 \pi / 4 \leqslant \varphi_{0} \leqslant \pi / 4, \\
\mathcal{G}_{j}\left(-\varphi_{0}+5 \pi / 4\right), & \pi / 4 \leqslant \varphi_{0} \leqslant 3 \pi / 4,\end{cases}
\end{gathered}
$$

and

$$
\delta= \begin{cases}0, & -3 \pi / 4 \leqslant \varphi_{0} \leqslant \pi / 4 \\ \delta_{*}, & \pi / 4 \leqslant \varphi_{0} \leqslant 3 \pi / 4\end{cases}
$$

The determination of the four constants completes the procedure that constructs the exact formulae for the spectral functions $\mathcal{S}_{1}(s), \mathcal{S}_{2}(s)$. The function $\mathcal{S}_{1}(s)$ is analytic everywhere in the strip $-3 \pi / 4 \leqslant \operatorname{Re} s \leqslant 3 \pi / 4$ apart from the pole $s=\varphi_{0}$. It decays exponentially at infinity as $e^{-|\operatorname{Im} s| / 3}$. The second function $\mathcal{S}_{2}(s)$ is analytic in the strip $-\pi / 4 \leqslant \operatorname{Re} s \leqslant \pi / 4$. It is bounded at infinity and $\mathcal{S}_{2}(i \infty)=-\mathcal{S}_{2}(-i \infty)$. In the exterior of the above strips both functions are meromorphic and single-valued. We have also established that if the parameter $\theta$ is real and the incident angle $\varphi_{0}$ equals $-\pi / 4-\theta$, then a non-physical reflected wave is observed in the shadow domain. For $\varphi_{0}=-\pi / 4+\theta$ or $\varphi_{0}=3 \pi / 4-\theta(\theta$ is real $)$, the reflected and surface coefficients become infinite.

\section{Case $\eta=0$ : no branch points}

In this section we aim to find a solution of equation (3.10) in the case when there are no branch points, and solving the Riemann-Hilbert problem on the elliptic surface can be bypassed.

\subsection{General solution of the auxiliary equation}

If $\eta=0$, then $\sin \theta=\frac{1}{2} \sqrt{3}$,

$$
a(s)=\cos ^{2} s-\frac{3}{4}, \quad b(s)=\cos ^{2} s-\frac{3}{2}, \quad \Delta(s)=3 \cos ^{2} s \sin ^{2} s,
$$

and the eigenvalues $\lambda_{1}, \lambda_{2}$ become

$$
\lambda_{1}(s)=\frac{\cos s+\sqrt{3} \sin s}{\cos s-\sqrt{3} \sin s}, \quad \lambda_{2}(s)=\frac{1}{\lambda_{1}(s)} .
$$


The matrix of transformation $\mathbf{T}(s)$ is single-valued, and the functions $F_{j}(z)=\phi_{j}(s) \quad(j=1,2)$, $z=i \cot \frac{1}{2} s$, are solutions to the following scalar Riemann-Hilbert problems:

$$
F_{j}^{+}(t)=l_{j}(t) F_{j}^{-}(t), \quad t \in(-1,1), \quad j=1,2,
$$

where

$$
l_{j}(t)=\lambda_{j}\left(\pi+i \log \frac{1+t}{1-t}\right)=\frac{1+t^{2}-(-1)^{j} 2 i \sqrt{3} t}{1+t^{2}+(-1)^{j} 2 i \sqrt{3} t}, \quad j=1,2
$$

In view of

$$
\lambda_{1}(\pi \pm i \infty)=-\frac{1}{2} \pm \frac{\sqrt{3}}{2} i, \quad \lambda_{2}(\pi \pm i \infty)=-\frac{1}{2} \mp \frac{\sqrt{3}}{2} i, \quad \lambda_{j}(\pi)=1,
$$

and since $\operatorname{Im} \lambda_{j}(\sigma), \sigma \in \Omega$, vanishes if and only if $s=\pi$, the increments of the arguments of the eigenvalues $\lambda_{1}, \lambda_{2}$ are equal to $\frac{4}{3} \pi,-\frac{4}{3} \pi$, respectively. Choose the branches of the functions $\log l_{j}(t)$ as follows:

$$
\arg l_{1}(-1)=-\frac{2 \pi}{3}, \quad \arg l_{2}(-1)=\frac{2 \pi}{3} .
$$

Then, obviously,

$$
\arg l_{1}(1)=\frac{2 \pi}{3}, \quad \arg l_{2}(1)=-\frac{2 \pi}{3} .
$$

The general solutions to the problems (7.3) have the form $F_{j}(z)=F_{j 0}(z) R_{j}(z)$, where $R_{j}(z)$, $j=1,2$, are rational functions, and

$$
F_{j 0}(z)=\exp \left\{\frac{1}{2 \pi i} \int_{-1}^{1} \frac{\log l_{j}(t)}{t-z} d t\right\}, \quad z \in \mathbb{C} \backslash[-1,1], \quad j=1,2 .
$$

Because of the relations (7.4), (7.6) and (7.7)

$$
\begin{gathered}
\log l_{j}(-t)=-\log l_{j}(t), \quad t \in[-1,1], \quad j=1,2, \\
\log l_{2}(t)=-\log l_{1}(t), \quad t \in[-1,1] .
\end{gathered}
$$

Therefore,

$$
F_{10}(z)=\exp \left\{\frac{1}{\pi i} \int_{0}^{1} \frac{t \log l_{1}(t)}{t^{2}-z^{2}} d t\right\}, \quad F_{20}(z)=\frac{1}{F_{1}(z)}, \quad z \in \mathbb{C} \backslash[-1,1],
$$

are even functions. At the end points,

$$
F_{10}(z)=O\left\{(z \mp 1)^{1 / 3}\right\}, \quad F_{20}(z)=O\left\{(z \mp 1)^{-1 / 3}\right\}, \quad z \rightarrow \pm 1 .
$$

The general solution of the auxiliary equation (3.10) when there are no branch points becomes

$$
f(s)=\Phi_{10}(s) P_{1}(s)+\Phi_{20}(s) P_{2}(s),
$$

where

$$
\Phi_{10}(s)=F_{10}(z), \quad \Phi_{20}(s)=\frac{1}{\Phi_{10}(s)}, \quad P_{j}(s)=R_{j}(z), \quad z=i \cot \frac{s}{2} .
$$

The functions $\Phi_{j 0}(s)$ are even, and $P_{j}(s)(j=1,2)$ are $2 \pi$-periodic meromorphic functions. 


\subsection{General solution of the governing equation (3.7)}

Define now which conditions have to satisfy the functions $P_{j}(s), j=1,2$, in order that formula (7.12) provides the general solution of equation (3.7). As in the general case, we substitute the general solution (7.12) of the auxiliary equation into equation (3.7)

$$
\begin{array}{r}
(\cos s-\sin \theta)\left\{\left[\Phi_{10}(s+\pi)+\Phi_{10}(s-\pi)\right] P_{1}(s+\pi)+\left[\Phi_{20}(s+\pi)+\Phi_{20}(s-\pi)\right] P_{2}(s+\pi)\right\} \\
=\cos s\left[\Phi_{10}(s) P_{1}(s)+\Phi_{20}(s) P_{2}(s)\right]
\end{array}
$$

and assume first that $\operatorname{Re} s=0$. By the mapping $z=i \cot s / 2$, a point $s$ of the imaginary axis is transformed into the point $x=i \cot s / 2 \in(-\infty,-1) \cup(1, \infty)$. As for the boundary points $s \pm \pi$, they fall into the points $(1 / x)^{ \pm} \in(-1,1)^{ \pm}$on the upper and lower banks of the cut $[-1,1]$. Therefore

$$
\Phi_{10}(s)=F_{10}(x)=\exp \chi_{1}(x), \quad \chi_{1}(x)=\frac{1}{2 \pi i} \int_{-1}^{1} \frac{\log l_{1}(t) d t}{t-x}, \quad x=i \cot \frac{s}{2} .
$$

By the Sokhotski-Plemelj formulae,

$$
\Phi_{10}(s \pm \pi)=F_{10}^{ \pm}\left(\frac{1}{x}\right)=\left[l_{1}(x)\right]^{ \pm 1 / 2} \exp \chi_{*}(x)
$$

where $\chi_{*}(x)$ is the principal value of the Cauchy integral

$$
\chi_{*}(x)=\frac{1}{2 \pi i} \int_{-1}^{1} \frac{\log l_{1}(t) d t}{t-1 / x} .
$$

On replacing $t$ by $1 / t$ and using the identity $l_{1}(1 / x)=l_{1}(x)$, we obtain another formula for the integral $\chi_{*}(x)$ :

$$
\chi_{*}(x)=\frac{1}{2 \pi i}\left(\int_{-\infty}^{-1}+\int_{1}^{\infty}\right)\left(\frac{1}{t}-\frac{1}{t-x}\right) \frac{\log l_{1}(t) d t}{t-x} .
$$

The next step of the procedure is to establish a relation between the functions $\chi_{1}(x)$ and $\chi_{*}(x)$. From (7.15) and (7.18),

$$
\chi_{1}(x)-\chi_{*}(x)=\frac{1}{2 \pi i} \int_{-\infty}^{\infty} \frac{\log l_{1}(t) d t}{t-x}-C,
$$

where the constant $C$ is given by

$$
C=\frac{1}{2 \pi i}\left(\int_{-\infty}^{-1}+\int_{1}^{\infty}\right) \frac{\log l_{1}(t) d t}{t} .
$$

To evaluate the integral in (7.19) consider the function

$$
g(z)=\frac{\log l_{1}(z)}{z-x}, \quad z \in(-\infty, \infty) .
$$




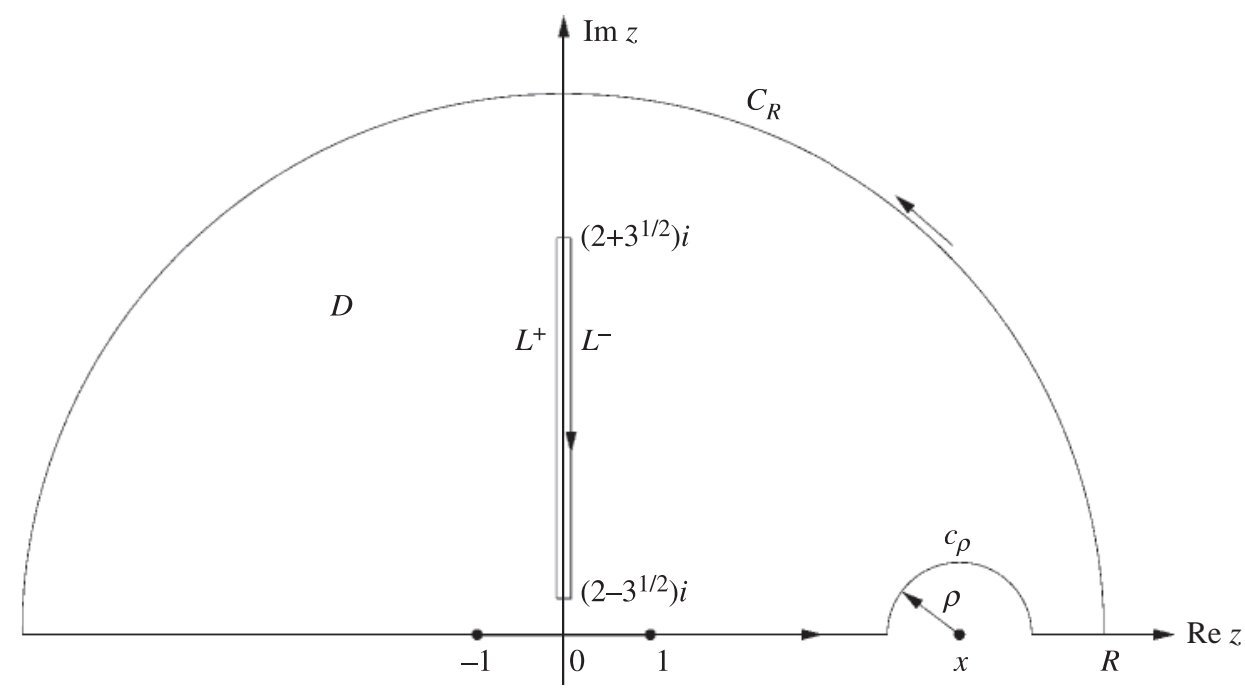

Fig. 5 The domain $D$ and the contour of integration: $\eta=0$

Since

$$
\log l_{1}(z)=\log \frac{1+z^{2}+2 i \sqrt{3} z}{1+z^{2}-2 i \sqrt{3} z}=\log \frac{[z-(2-\sqrt{3}) i][z+(2+\sqrt{3}) i]}{[z-(2+\sqrt{3}) i][z+(2-\sqrt{3}) i]}, \quad \log l_{1}(\infty)=0,
$$

it follows that the function $g(z)$ is analytic in the $z$-plane cut along the segments $[-(2+\sqrt{3}) i,-(2-$ $\sqrt{3}) i]$ and $[(2-\sqrt{3}) i,(2+\sqrt{3}) i]$ of the imaginary axis, apart from the simple pole at the point $z=x$. As $z \rightarrow \infty, g(z) \sim 4 i \sqrt{3} z^{-2}$. To apply the Cauchy theorem to the function $g(z)$ analytic in the domain $D$ (Fig.5), we notice that

$$
\begin{gathered}
\int_{c_{\rho}} g(z) d z=-\pi i \log l_{1}(x)+\rho \mathcal{I}(\rho), \quad|\mathcal{I}(\rho)| \leqslant K_{0}, \quad K_{0}=\text { const }, \\
\int_{C_{R}} g(z) d z=R^{-1} \mathcal{I}^{\prime}(R), \quad\left|\mathcal{I}^{\prime}(R)\right| \leqslant K_{0}^{\prime}, \quad K_{0}^{\prime}=\text { const }
\end{gathered}
$$

and

$$
\int_{L^{+} \cup L^{-}} g(z) d z=2 \pi i \log \frac{x-(2-\sqrt{3}) i}{x-(2+\sqrt{3}) i}
$$

On letting $\rho \rightarrow 0$ and $R \rightarrow \infty$, we finally obtain

$$
\frac{1}{2 \pi i} \int_{-\infty}^{\infty} \frac{\log l_{1}(t) d t}{t-x}=\frac{1}{2} \log \frac{[x-(2+\sqrt{3}) i][x+(2+\sqrt{3}) i]}{[x-(2-\sqrt{3}) i][x+(2-\sqrt{3}) i]} .
$$


On applying this result it is possible to evaluate the constant $C$. The identity $\log l_{1}(1 / t)=\log l_{1}(t)$ and formula (7.20) imply

$$
C=\frac{1}{2 \pi i} \int_{-1}^{1} \frac{\log l_{1}(t) d t}{t}
$$

By adding (7.20) and (7.26) and setting $x=0$ in (7.25) we find

$$
C=\log (2+\sqrt{3})
$$

Substituting formulae (7.25) and (7.27) into (7.19) yields the desired relation

$$
\chi_{*}(x)=\chi_{1}(x)+\log (2+\sqrt{3})-\frac{1}{2} \log \frac{[x-(2+\sqrt{3}) i][x+(2+\sqrt{3}) i]}{[x-(2-\sqrt{3}) i][x+(2-\sqrt{3}) i]} .
$$

On using (7.15), (7.16) and (7.28), we observe that the limiting values of the functions $\Phi_{j 0}(s \pm \pi)$, $j=1,2$, have the following representations, which are required in the sequel:

$$
\begin{aligned}
& \Phi_{10}(s \pm \pi)=(2+\sqrt{3}) \frac{x \mp(2-\sqrt{3}) i}{x \mp(2+\sqrt{3}) i} \Phi_{10}(s), \\
& \Phi_{20}(s \pm \pi)=(2-\sqrt{3}) \frac{x \mp(2+\sqrt{3}) i}{x \mp(2-\sqrt{3}) i} \Phi_{20}(s) .
\end{aligned}
$$

Consequently, by using the relations

$$
\begin{aligned}
x^{2} & =\frac{\cos s+1}{\cos s-1}, \\
\Phi_{j 0}(s+\pi)+\Phi_{j 0}(s-\pi) & =\frac{2 \cos s \Phi_{j 0}(s)}{2 \cos s+(-1)^{j} \sqrt{3}}, \quad j=1,2
\end{aligned}
$$

we are able to replace equation (7.14) by

$$
\Phi_{10}(s) P_{1}(s+\pi)+\frac{2 \cos s-\sqrt{3}}{2 \cos s+\sqrt{3}} \Phi_{20}(s) P_{2}(s+\pi)=\Phi_{10}(s) P_{1}(s)+\Phi_{20}(s) P_{2}(s), \quad \operatorname{Re} s=0 .
$$

Because of the linear independence of the functions $\Phi_{10}(s)$ and $\Phi_{20}(s)$ we obtain

$$
\begin{aligned}
P_{1}(s+\pi) & =P_{1}(s) \\
(2 \cos s-\sqrt{3}) P_{2}(s+\pi) & =(2 \cos s+\sqrt{3}) P_{2}(s) .
\end{aligned}
$$

By the uniqueness theorem, these relations are valid not just on the imaginary axis but also in the whole $s$-plane. Thus, if the $2 \pi$-periodic meromorphic functions $P_{1}(s), P_{2}(s)$ meet the requirements (7.32), then the function $f(s)$ given by (7.12) is not only a solution of the auxiliary equation (3.10) but also the general solution of equation (3.7) in the strip $-\pi \leqslant \operatorname{Re} s \leqslant \pi$.

We next specify the behaviour of the meromorphic functions at infinity and their possible poles. From (7.11),

$$
\Phi_{10}(s)=O\left(e^{-|\operatorname{Im} s| / 3}\right), \quad \Phi_{20}(s)=O\left(e^{|\operatorname{Im} s| / 3}\right), \quad \operatorname{Im} s \rightarrow \pm \infty .
$$


Therefore the functions $P_{1}(s)$ and $P_{2}(s)$ may grow at infinity and

$$
\left|P_{1}(s)\right| \leqslant K_{1} e^{\frac{7}{3}|\operatorname{Im} s|}, \quad\left|P_{2}(s)\right| \leqslant K_{2} e^{\frac{5}{3}|\operatorname{Im} s|}, \quad \operatorname{Im} s \rightarrow \pm \infty,
$$

where $K_{1}, K_{2}$ are non-zero constants. Similarly to the general case, we outline the set of potential poles of the meromorphic functions $P_{1}(s), P_{2}(s)$. They are the zeros of the function $\Delta^{1 / 2}(s)=$ $\sqrt{3} \cos s \sin s$ and also the points $\pm \frac{5}{6} \pi+2 \pi n, n \in \mathbb{Z}\left(\theta=\frac{1}{3} \pi\right)$. However, the assumption that $s=\frac{5}{6} \pi$ is a pole of the function $P_{1}(s)$ conflicts with the first relation (7.32). This is because the function $P_{1}(s+\pi)$ is bounded as $s=\frac{5}{6} \pi$. The point $s=\frac{5}{6} \pi$ cannot be a pole of the function $P_{2}(s)$ for the function $f(s)$ being analytic at the point $s=\frac{5}{6} \pi$. Next, the functions $P_{1}(s)$ and $P_{2}(s)$ are $2 \pi$-periodic and odd. Therefore they are analytic at all the points $\pm \frac{5}{6} \pi+2 \pi n, n \in \mathbb{Z}$. So, they may have simple poles at the zeros of the function $\sin 2 s$ only.

The most general form of the described functions is

$$
P_{1}(s)=\frac{1}{\sin 2 s} \sum_{n=0}^{4} E_{n}^{\prime} \cos n s, \quad P_{2}(s)=\frac{1}{\sin 2 s} \sum_{n=0}^{3} E_{n}^{\prime \prime} \cos n s,
$$

where $E_{n}^{\prime}$ and $E_{n}^{\prime \prime}$ are arbitrary constants. Satisfying the conditions (7.32) yields $E_{1}^{\prime}=E_{3}^{\prime}=0$, $E_{1}^{\prime \prime}=-\sqrt{3} E_{0}^{\prime \prime}, E_{2}^{\prime \prime}=E_{0}^{\prime \prime}, E_{3}^{\prime \prime}=0$. Hence

$$
P_{1}(s)=\frac{E_{0}^{\prime}+E_{2}^{\prime} \cos 2 s+E_{4}^{\prime} \cos 4 s}{\sin 2 s}, \quad P_{2}(s)=\frac{E_{0}^{\prime \prime}(2 \cos s-\sqrt{3})}{2 \sin s} .
$$

Because of the analyticity of the function $f(s)$ in the strip $-\pi \leqslant \operatorname{Re} s \leqslant \pi$ we need to make sure that

$$
\underset{s=0}{\text { res }} f(s)=0, \quad \underset{s=\pi / 2}{\text { res }} f(s)=0, \quad \underset{s=\pi}{\text { res }} f(s)=0 .
$$

On using that $\Phi_{10}(0)=\Phi_{20}(0)=1$, we find from the first two conditions

$$
E_{0}^{\prime}+E_{2}^{\prime}+E_{4}^{\prime}+(2-\sqrt{3}) E_{0}^{\prime \prime}=0, \quad E_{2}^{\prime}=E_{0}^{\prime}+E_{4}^{\prime} .
$$

From the relations $\Phi_{10}(\pi)=2+\sqrt{3}, \Phi_{20}(\pi)=(2+\sqrt{3})^{-1}$ and also (7.38) it follows that the third condition in (7.37) is satisfied automatically. Finally, the general solution of equation (3.7) in the chosen class becomes

$$
f(s)=\frac{B_{0}+B_{1} \cos 2 s+\left(B_{1}-B_{0}\right) \cos 4 s}{\sin 2 s} \Phi_{10}(s)-\frac{(2+\sqrt{3}) B_{1}(2 \cos s-\sqrt{3})}{\sin s \Phi_{10}(s)},
$$

where we introduced the new constants $B_{0}=E_{0}^{\prime}$ and $B_{1}=E_{2}^{\prime}$. So, as in the case $\eta \neq 0$, there are two arbitrary constants $B_{0}$ and $B_{1}$ in the representation of the function $f(s)$. To adjust the procedure of section 6.2 for the constants $B_{j}$ and $C_{j}(j=0,1)$ to the case $\eta=0$, we rewrite the expression (7.39) for the function $f(s)$ in the form $f(s)=B_{0} \mathcal{F}_{0}(s)+B_{1} \mathcal{F}_{1}(s)$, where

$$
\begin{gathered}
\mathcal{F}_{0}(s)=2 \sin 2 s \Phi_{10}(s), \\
\mathcal{F}_{1}(s)=\frac{\cos 2 s+\cos 4 s}{\sin 2 s} \Phi_{10}(s)-\frac{(2+\sqrt{3})(2 \cos s-\sqrt{3})}{\sin s \Phi_{10}(s)} .
\end{gathered}
$$


We emphasize that the above functions are bounded at $s=0\left(\mathcal{F}_{j}(0)=0\right)$ and they are free of the constants $B_{0}, B_{1}$. Then the four unknown constants can be found by the method of section 6.2 by formulae (6.21).

Finally, we note that the spectral functions $\mathcal{S}_{1}(s), \mathcal{S}_{2}(s)$ defined by (3.17), (3.18) have the same asymptotics at infinity as in the case $\eta \neq 0$.

\section{High frequency asymptotics}

\subsection{Reflected, transmitted and surface waves}

As $k_{0} r \rightarrow \infty$, the electric field $E_{z}$ can be represented as follows:

$$
E_{z} \sim E_{z}^{i}+E_{z}^{r}+E_{z}^{t}+E_{z}^{s}+E_{z}^{d}
$$

where $E_{z}^{i}, E_{z}^{r}, E_{z}^{t}, E_{z}^{s}$ and $E_{z}^{d}$ are the incident, reflected, transmitted, surface and diffracted waves, respectively. In this section we define these waves for the wedges $-3 \pi / 4<\varphi<3 \pi / 4$ and $3 \pi / 4<\varphi<5 \pi / 4$. The usual trick of constructing the above waves is to apply the method of steepest descent and to deform the contour $\Gamma$ into another one consisting of two steepest descent paths. The right-hand path is given by $\operatorname{Re} s=\pi+\operatorname{gd}(\operatorname{Im} s) \operatorname{sgn} \operatorname{Im} s$, where $\operatorname{gd} x$ is the Gudermann function $\operatorname{gd} x=\arccos (1 / \cosh x)$. This curve goes from the infinite point $s=\pi / 2-i \infty$, crosses the real axis at the point $s=\pi$ and then travels to the upper infinite point $s=3 \pi / 2+i \infty$. The lines $\operatorname{Re} s=\pi / 2$ and $\operatorname{Re} s=3 \pi / 2$ are the asymptotes for the lower and the upper part of the path, respectively. The second path is symmetric to the first one with respect to the origin.

Let first the point of observation be a point in the external wedge $-3 \pi / 4<\varphi<3 \pi / 4$. Analysis of the first spectral function $\mathcal{S}_{1}(s+\varphi)$ and the conditions (6.12) shows that the geometrical optics poles should be among the ones listed below:

$$
\begin{gathered}
s=-\varphi+\varphi_{0}, \quad \max \left\{-3 \pi / 4, \varphi_{0}-\pi\right\}<\varphi<\min \left\{3 \pi / 4, \varphi_{0}+\pi\right\}, \quad-3 \pi / 4<\varphi_{0}<3 \pi / 4, \\
s=-\varphi+\varphi_{0}+\pi, \quad \varphi_{0}<\varphi<3 \pi / 4, \quad-\pi / 4<\varphi_{0}<3 \pi / 4, \\
s=-\varphi+\varphi_{0}-\pi, \quad-3 \pi / 4<\varphi<\varphi_{0}, \quad-3 \pi / 4<\varphi_{0}<\pi / 4, \\
s=-\varphi-\varphi_{0}+\pi / 2, \quad-\pi / 2-\varphi_{0}<\varphi<3 \pi / 4, \quad-3 \pi / 4<\varphi_{0}<-\pi / 4, \\
s=-\varphi-\varphi_{0}-\pi / 2, \quad-3 \pi / 4<\varphi<\pi / 2-\varphi_{0}, \quad \pi / 4<\varphi_{0}<3 \pi / 4, \\
s=-\varphi-\varphi_{0}+3 \pi / 2, \quad \pi / 2-\varphi_{0}<\varphi<3 \pi / 4, \quad-\pi / 4<\varphi_{0}<3 \pi / 4, \\
s=-\varphi-\varphi_{0}-3 \pi / 2, \quad-3 \pi / 4<\varphi<-\pi / 2-\varphi_{0}, \quad-3 \pi / 4<\varphi_{0}<\pi / 4, \\
s=-\varphi+\varphi_{0}+2 \pi, \quad \pi+\varphi_{0}<\varphi<3 \pi / 4, \quad-3 \pi / 4<\varphi_{0}<-\pi / 4, \\
s=-\varphi+\varphi_{0}-2 \pi, \quad-3 \pi / 4<\varphi<\varphi_{0}-\pi, \quad \pi / 4<\varphi_{0}<3 \pi / 4 .
\end{gathered}
$$

To evaluate the residues of the function $\mathcal{S}_{1}(s)$ at the above poles we need to continue the function $\mathcal{S}_{1}(s)$ into the strip $5 \pi / 4 \leqslant \operatorname{Re} s \leqslant 9 \pi / 4$ :

$$
\mathcal{S}_{1}(s)=\Sigma(s)\left[\psi\left(s-\frac{5 \pi}{4}\right)-f\left(s-\frac{9 \pi}{4}\right)+\frac{\cos (s-\pi / 4) f(s-5 \pi / 4)}{\cos (s-\pi / 4)+\sin \theta}\right] .
$$


On using formulae (6.15) to (6.18) we deduce the following result. If $-3 \pi / 4<\varphi_{0}<-\pi / 4$, then

$$
\begin{aligned}
& E_{z}^{i}=e^{i k_{0} r \cos \left(\varphi-\varphi_{0}\right)} \omega_{\varphi}\left(-\frac{3 \pi}{4}, \pi+\varphi_{0}\right), E_{z}^{t}=e^{i k_{0} r \cos \left(\varphi-\varphi_{0}\right)}\left[1-f\left(\varphi_{0}-\frac{\pi}{4}\right)\right] \omega_{\varphi}\left(\pi+\varphi_{0}, \frac{3 \pi}{4}\right), \\
& E_{z}^{r}=-e^{i k_{0} r \sin \left(\varphi+\varphi_{0}\right)}\left\{\omega_{\varphi}\left(-\frac{3 \pi}{4},-\frac{\pi}{2}-\varphi_{0}\right)+\left[1-f\left(\varphi_{0}-\frac{\pi}{4}\right)\right] \omega_{\varphi}\left(-\frac{\pi}{2}-\varphi_{0}, \frac{3 \pi}{4}\right)\right\},
\end{aligned}
$$

where

$$
\omega_{\varphi}(a, b)= \begin{cases}1, & \varphi \in[a, b], \\ 0, & \varphi \notin[a, b] .\end{cases}
$$

In view of (6.15), for all $\varphi_{0}$ apart from $\varphi_{0}=-\pi / 4-\theta$ ( $\theta$ is real), the value $f\left(\varphi_{0}-\pi / 4\right)$ has to be 1 . The assumption that $f\left(\varphi_{0}-\pi / 4\right) \neq 1$ in the case $\delta_{+}=0$ gives reflected waves in the sector $-\pi / 2-\varphi_{0}<\varphi<3 \pi / 4$; that is impossible. Therefore, for all $\varphi_{0}, f\left(\varphi_{0}-\pi / 4\right)=1$, and the transmitted and reflected waves in (8.4) have the form

$$
E_{z}^{t}=0, \quad E_{z}^{r}=-e^{i k_{0} r \sin \left(\varphi+\varphi_{0}\right)} \omega_{\varphi}\left(-3 \pi / 4,-\pi / 2-\varphi_{0}\right) .
$$

In the case $-\pi / 4<\varphi_{0}<\pi / 4$ the incident, transmitted and reflected waves are

$$
\begin{gathered}
E_{z}^{i}=e^{i k_{0} r \cos \left(\varphi-\varphi_{0}\right)}, \quad E_{z}^{t}=0, \\
E_{z}^{r}=-\delta_{*} e^{-i k_{0} r \sin \left(\varphi+\varphi_{0}\right)} \omega_{\varphi}\left(\pi / 2-\varphi_{0}, 3 \pi / 4\right)-e^{i k_{0} r \sin \left(\varphi+\varphi_{0}\right)} \omega_{\varphi}\left(-3 \pi / 4,-\pi / 2-\varphi_{0}\right) .
\end{gathered}
$$

In the last possible case $\pi / 4<\varphi_{0}<3 \pi / 4$, the waves have the form

$$
\begin{gathered}
E_{z}^{i}=e^{i k_{0} r \cos \left(\varphi-\varphi_{0}\right)} \omega_{\varphi}\left(\varphi_{0}-\pi, 3 \pi / 4\right), \quad E_{z}^{t}=0, \\
E_{z}^{r}=-\delta_{*} e^{-i k_{0} r \sin \left(\varphi+\varphi_{0}\right)} \omega_{\varphi}\left(\pi / 2-\varphi_{0}, 3 \pi / 4\right) .
\end{gathered}
$$

Here $\delta_{*}$ is the same parameter as in (6.18). In the singular case, when $\delta_{-}=0$ we have $\theta$ is real and $\varphi_{0}=\theta-\pi / 4$ if $-\pi / 4<\varphi_{0}<\pi / 4$ and $\varphi_{0}=-\theta+3 \pi / 4$ if $\pi / 4<\varphi_{0}<3 \pi / 4$. In both cases the reflected coefficient $-\delta_{*}$ is infinite.

On using the conditions (6.12), we next write the poles of the function $\mathcal{S}_{2}(s)$ which may correspond to the reflected and transmitted waves in the sector $3 \pi / 4<\varphi<5 \pi / 4$ :

$$
\begin{gathered}
s=-\varphi+\varphi_{0}+\pi, \quad 3 \pi / 4<\varphi<5 \pi / 4, \quad \pi / 4<\left|\varphi_{0}\right|<3 \pi / 4, \\
s=-\varphi-\varphi_{0}+3 \pi / 2, \quad \max \left\{3 \pi / 4, \pi / 2-\varphi_{0}\right\}<\varphi<5 \pi / 4, \quad-3 \pi / 4<\varphi_{0}<\pi / 4, \\
s=-\varphi-\varphi_{0}+\pi / 2, \quad 3 \pi / 4<\varphi<\min \left\{3 \pi / 2-\varphi_{0}, 5 \pi / 4\right\}, \quad-\pi / 4<\varphi_{0}<3 \pi / 4, \\
s=-\varphi+\varphi_{0}+2 \pi, \quad \max \left\{3 \pi / 4, \pi+\varphi_{0}\right\}<\varphi<5 \pi / 4, \quad-3 \pi / 4<\varphi_{0}<\pi / 4, \\
s=-\varphi+\varphi_{0}, \quad 3 \pi / 4<\varphi<\min \left\{\pi+\varphi_{0}, 5 \pi / 4\right\}, \quad-\pi / 4<\varphi_{0}<3 \pi / 4, \\
s=-\varphi-\varphi_{0}+5 \pi / 2, \quad 3 \pi / 2-\varphi_{0}<\varphi<5 \pi / 4, \quad \pi / 4<\varphi_{0}<3 \pi / 4, \\
s=-\varphi-\varphi_{0}-\pi / 2, \quad 3 \pi / 4<\varphi<\pi / 2-\varphi_{0}, \quad-3 \pi / 4<\varphi<-\pi / 4 .
\end{gathered}
$$

As in the case of the angular region $|\varphi|<3 \pi / 4$, to evaluate the residues at these poles in addition 
to formulae (3.18) we need the relevant spectral function in the strips $\pi / 4 \leqslant \operatorname{Re} s \leqslant 5 \pi / 4$ and $-7 \pi / 4 \leqslant \operatorname{Re} s \leqslant-3 \pi / 4$. By analytical continuation of (3.18) to the left and to the right we obtain

$$
\begin{aligned}
\mathcal{S}_{2}(s)= & \Sigma(s)\left\{\left(1-\frac{\sin (s+\pi / 4)}{\sin \theta}\right) \psi\left(s+\frac{7 \pi}{4}\right)-\frac{\sin (s+\pi / 4)}{\sin \theta}\right. \\
& \left.\times\left[\psi\left(s+\frac{3 \pi}{4}\right)+f\left(s+\frac{7 \pi}{4}\right)-\frac{\sin (s+\pi / 4) f(s+3 \pi / 4)}{\sin (s+\pi / 4)+\sin \theta}\right]\right\}, \quad-\frac{7 \pi}{4} \leqslant \operatorname{Re} s \leqslant-\frac{3 \pi}{4}, \\
\mathcal{S}_{2}(s)= & \Sigma(s)\left\{\left(1-\frac{\sin (s+\pi / 4)}{\sin \theta}\right)\left[\psi\left(s-\frac{\pi}{4}\right)-f\left(s-\frac{5 \pi}{4}\right)\right]\right. \\
& \left.-\frac{\sin (s+\pi / 4)}{\sin \theta}\left[f\left(s-\frac{\pi}{4}\right)+\psi\left(s-\frac{5 \pi}{4}\right)\right]\right\}, \quad \frac{\pi}{4} \leqslant \operatorname{Re} s \leqslant \frac{5 \pi}{4} .
\end{aligned}
$$

Because of the relations (6.15) and (6.17) not all the residues of the function $\mathcal{S}_{2}(s)$ at the poles (8.9) are non-zero. The reflected and transmitted waves can be written straightforwardly $\left(E_{z}^{i}=0\right)$ :

$$
\begin{gathered}
E_{z}^{r}=0, \quad E_{z}^{t}=0, \quad-3 \pi / 4<\varphi_{0}<-\pi / 4, \\
E_{z}^{r}=0, \quad E_{z}^{t}=\frac{\sin \theta}{\delta_{-}} \omega_{\varphi}\left(\frac{3 \pi}{4}, \pi+\varphi_{0}\right) e^{i k_{0} r \cos \left(\varphi-\varphi_{0}\right)}, \quad-\frac{\pi}{4}<\varphi_{0}<\frac{\pi}{4}, \\
E_{z}^{r}=-\frac{\sin \theta}{\delta_{-}} \omega_{\varphi}\left(\frac{3 \pi}{2}-\varphi_{0}, \frac{5 \pi}{4}\right) e^{i k_{0} r \sin \left(\varphi+\varphi_{0}\right)}, \quad E_{z}^{t}=\frac{\sin \theta}{\delta_{-}} e^{i k_{0} r \cos \left(\varphi-\varphi_{0}\right)}, \quad \frac{\pi}{4}<\varphi_{0}<\frac{3 \pi}{4} .
\end{gathered}
$$

The coefficient $\delta_{-}$is defined by (6.14) and it is assumed to be non-zero.

We now specify the poles of the function $\mathcal{S}_{1}(s)$ which define the surface waves in the sector $-3 \pi / 4<\varphi<3 \pi / 4$. Analysis of formulae (3.17) and (8.3) indicates that there is only one pole of the function $\mathcal{S}_{1}(s+\varphi)$ at the point $s=-\varphi-\theta+7 \pi / 4$ that gives rise to the surface wave

$$
E_{z}^{s}=W e^{i k_{0} r \cos (\pi / 4+\varphi+\theta)} \omega_{\varphi}(3 \pi / 4-\operatorname{Re} \theta+\operatorname{gd}(\operatorname{Im} \theta) \operatorname{sgn}(\operatorname{Im} \theta), 3 \pi / 4),
$$

where

$$
W=\frac{2 \cos 2 \varphi_{0} \tan \theta}{\cos 2 \theta+\sin 2 \varphi_{0}} f\left(\frac{\pi}{2}-\theta\right)
$$

Clearly, the surface coefficient $W$ is infinite if the incident angle $\varphi_{0}$ coincides with one of the singular values $-\pi / 4+\theta$, or $3 \pi / 4-\theta$. If $\varphi_{0}=-\pi / 4-\theta$, then $f(\pi / 2-\theta)=0$, and the coefficient $W$ is finite.

Let now $3 \pi / 4<\varphi<5 \pi / 4$. The surface wave pole of the function $\mathcal{S}_{2}(s+\varphi-\pi)$ is $s=$ $-\varphi+\theta-\pi / 4$. On evaluating the residue of the function $\mathcal{S}_{2}(s+\varphi-\pi)$ at this point, we find the surface wave

$$
E_{z}^{s}=-W e^{i k_{0} r \cos (\pi / 4+\varphi-\theta)} \omega_{\varphi}(3 \pi / 4,3 \pi / 4+\operatorname{Re} \theta-\operatorname{gd}(\operatorname{Im} \theta) \operatorname{sgn}(\operatorname{Im} \theta)),
$$

with the coefficient $W$ given by (8.13). 


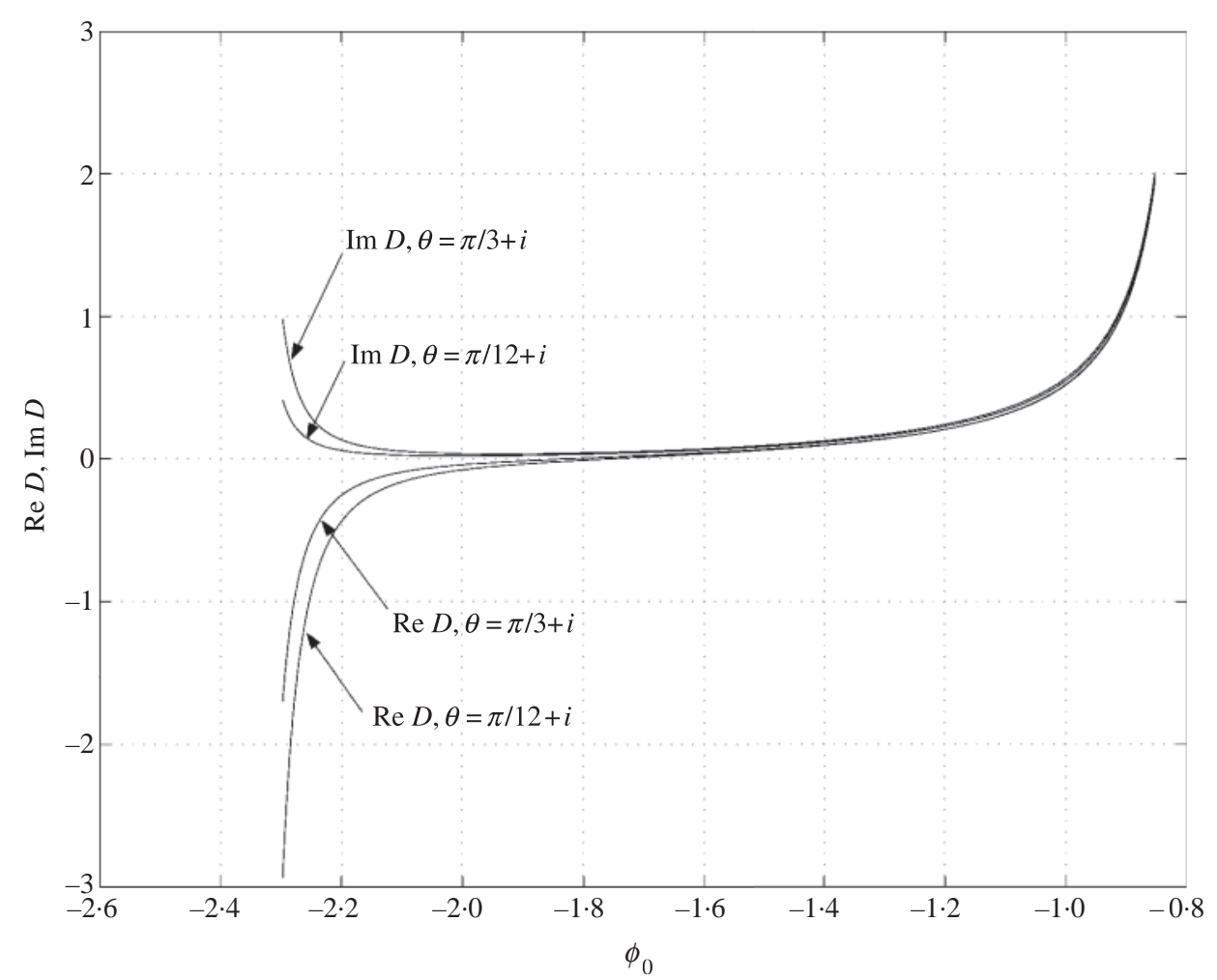

Fig. 6 The real and imaginary parts of the backscattering coefficient for $\varphi_{0} \in(-3 \pi / 4,-\pi / 4)$

\subsection{Diffracted field. Numerical results}

An important characteristic in the geometrical theory of diffraction is the diffraction coefficient (the scattering diagram). By applying the steepest descent method for $k_{0} r \gg 1$ (see, for example, Senior and Volakis (1)) we obtain the diffracted field

$$
E_{z}^{d}=\frac{e^{-i k_{0} r}}{\sqrt{k_{0} r}} D(\varphi), \quad \varphi \in(-3 \pi / 4,5 \pi / 4) \backslash\{\varphi=3 \pi / 4\},
$$

where

$$
\begin{gathered}
D(\varphi)= \begin{cases}D_{1}(\varphi), & -3 \pi / 4<\varphi<3 \pi / 4, \\
D_{2}(\varphi), & 3 \pi / 4<\varphi<5 \pi / 4,\end{cases} \\
D_{1}(\varphi)=\frac{e^{i \pi / 4}}{\sqrt{2 \pi}}\left[\mathcal{S}_{1}(\varphi-\pi)-\mathcal{S}_{1}(\varphi+\pi)\right], \quad D_{2}(\varphi)=\frac{e^{i \pi / 4}}{\sqrt{2 \pi}}\left[\mathcal{S}_{2}(\varphi-2 \pi)-\mathcal{S}_{2}(\varphi)\right] .
\end{gathered}
$$

To illustrate the method presented we consider the most interesting and complicated case $\eta \neq 0$. Clearly, to evaluate the above coefficients we need the spectral functions $\mathcal{S}_{1}(s)$ and $\mathcal{S}_{2}(s)$ given 


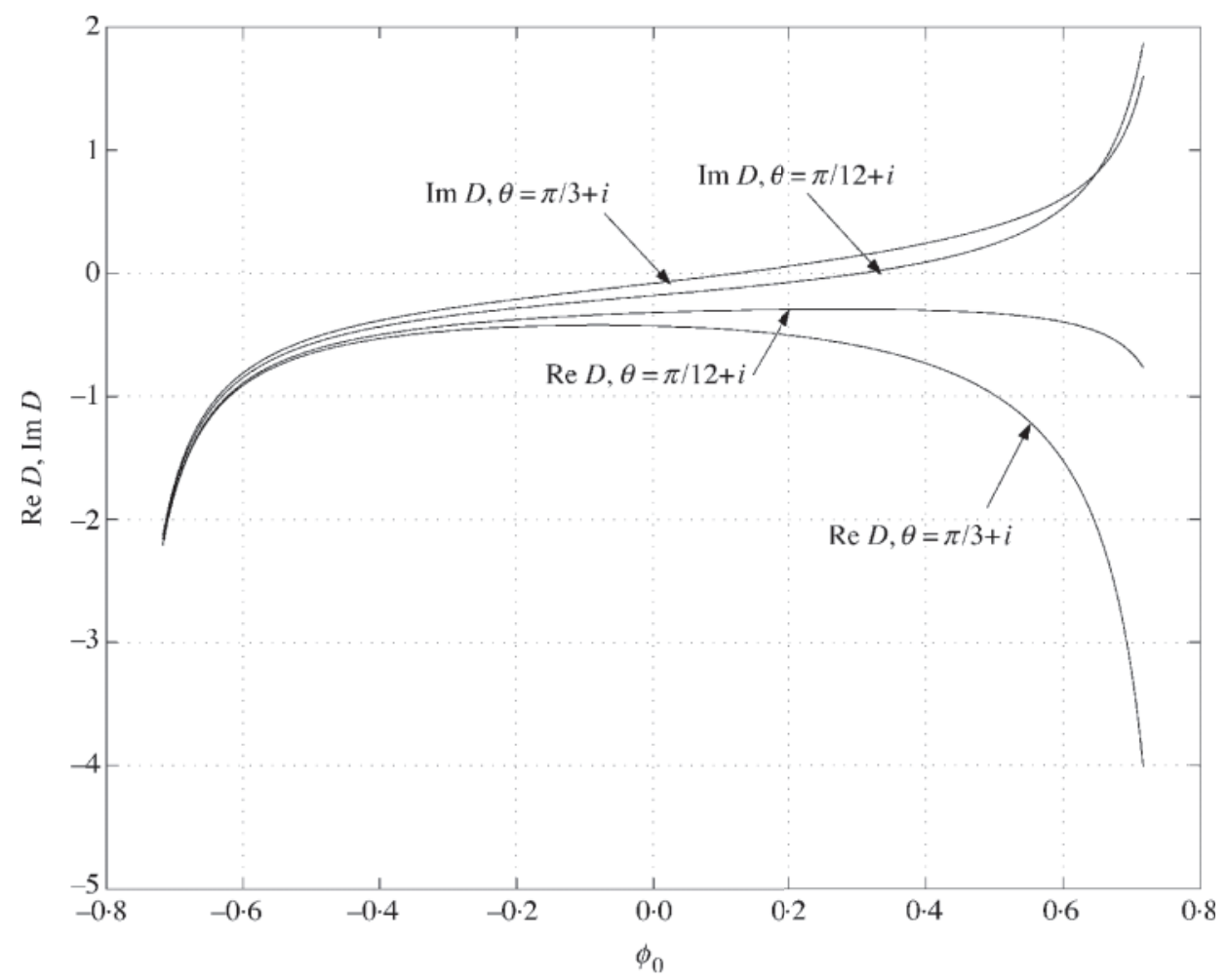

Fig. 7 The real and imaginary parts of the backscattering coefficient for $\varphi_{0} \in(-\pi / 4, \pi / 4)$

by (3.17), (3.18), (8.3) and (8.10). The functions $f(s), \psi(s)$ appearing in these formulae and the constants $B_{j}, C_{j}(j=0,1)$ are defined in terms of the functions $\mathcal{F}_{j}(s)$ and $\mathcal{G}_{j}(s)(j=0,1)$ by (6.19) to (6.22). Next, the functions $\mathcal{F}_{j}(s)$ and $\mathcal{G}_{j}(s)$ are found in terms of elementary functions and the three quadratures (4.63) and (6.20). Without loss of generality, we take the contour of integration for the second integral in (4.63) as the straight segment joining the points $t=1$ and $t=z_{1}$ with the starting point $t=1$. Let $\chi_{2}(z)=\mathcal{J}_{1}(z)+\mathcal{J}_{2}(z)$. The three integrals to be evaluated can be represented in the form

$$
\begin{gathered}
\mathcal{J}_{1}(z)=-\frac{z t_{1}}{\pi i} \int_{0}^{1} \frac{\left[\log l_{1}\left(t_{1} x\right)+\pi i\right] d x}{q^{1 / 2}\left(t_{1} x\right)\left(t_{1}^{2} x^{2}-z^{2}\right)}, \quad z \notin L, \\
\mathcal{J}_{1}^{+}(t)=\frac{\log l_{1}(t)+\pi i}{2 q^{1 / 2}(t)}+\mathcal{J}_{1}(t), \quad t \in L^{+} \quad(s \in \Omega), \\
\mathcal{J}_{2}(z)=-z\left(z_{1}-1\right) \int_{0}^{1} \frac{d x}{q^{1 / 2}\left(t^{+}\right)\left(t^{2}-z^{2}\right)}, \quad t=\left(z_{1}-1\right) x+1, \quad t^{+} \in\left(1, z_{1}\right)^{+}, \\
\mathcal{G}_{j}(s)=\frac{\cos s}{2 \pi} \int_{0}^{1} \frac{\mathcal{F}_{j}(\tau-\pi)}{1-x^{2}}[\Lambda(\tau, s)-\Lambda(-\tau, s)] d x, \quad \tau=-i \log \frac{x+1}{x-1}, \quad j=0,1 .
\end{gathered}
$$


Here $\mathcal{J}_{1}(t)$ is the principal value of the Cauchy integral $\mathcal{J}_{1}(z)$ on the contour $L$.

If the angle of observation $\varphi$ coincides with the angle of incident $\varphi_{0}$, then the corresponding diffracted field becomes the backscattering field. The backscattering coefficient

$$
D\left(\varphi_{0}\right)=\lim _{\varphi \rightarrow \varphi_{0}} D_{1}(\varphi)
$$

is evaluated on using (8.16) and (6.15) to (6.18):

$$
D\left(\varphi_{0}\right)=-\frac{e^{i \pi / 4}}{\sqrt{2 \pi}} f^{\prime}\left(\varphi_{0}+\frac{3 \pi}{4}\right), \quad-\frac{3 \pi}{4}<\varphi_{0}<\frac{\pi}{4},
$$

and

$$
\begin{aligned}
D\left(\varphi_{0}\right)= & \frac{e^{i \pi / 4}}{\sqrt{2 \pi}}\left[f^{\prime}\left(\varphi_{0}-\frac{5 \pi}{4}\right)+\frac{\sin \left(\varphi_{0}+\pi / 4\right)}{\delta_{-}} f^{\prime}\left(\varphi_{0}-\frac{\pi}{4}\right)\right. \\
& \left.+\frac{2 \delta_{-} \cos \left(\varphi_{0}+\pi / 4\right)+\cos 2 \varphi_{0}}{2 \delta_{-}^{2}}\right], \quad \frac{\pi}{4}<\varphi_{0}<\frac{3 \pi}{4} .
\end{aligned}
$$

Numerical computations are implemented for different values of the impedance parameter $\gamma$. If for instance $\theta=\pi / 3+i$, then the values for the parameters of interest become $\gamma=1.3363+$ $0.5876 i, \eta=0.4918-1.1592 i, t_{1}=0.5459+0.1794 i, 1 / k=-0.2658-0.1959 i, z_{1}=1.0800-$ $0.0917 i, z_{2}=-0.2291-0.2008 i$. In Figs 6 to 8 we present the real and imaginary parts of the backscattering coefficient $D\left(\varphi_{0}\right)$ for $\theta=\pi / 3+i$ and $\theta=\pi / 12+i$. As $\varphi_{0}$ tends to one of the critical values $\pm 3 \pi / 4, \pm \pi / 4$, the real and imaginary parts of the backscattering coefficient tend to infinity.

Let us analyse the case of large $\gamma$. If $\operatorname{Re} \theta \in(0, \pi / 2)$ and $|\operatorname{Im} \theta| \rightarrow \infty$, then $|\gamma| \rightarrow \infty$, and the magnetically conductive sheet $\varphi=3 \pi / 4$ vanishes since the boundary conditions (2.5) for $\gamma=\infty$ mean the continuity of the field $E_{z}$ and its normal derivative through the line $\varphi=3 \pi / 4$. In this elementary case the spectral functions can be found in terms of elementary functions

$$
\mathcal{S}_{1}(s)=\frac{\sin \left(\varphi_{0} / 2+3 \pi / 8\right)}{2\left[\cos \left(\varphi_{0} / 2+3 \pi / 8\right)-\cos (s / 2+3 \pi / 8)\right]}, \quad \mathcal{S}_{2}(s)=\mathcal{S}_{1}(s+\pi),
$$

and the backscattering coefficient is given by

$$
D_{\infty}\left(\varphi_{0}\right)=-\frac{e^{i \pi / 4} \cos ^{2}\left(\varphi_{0} / 2-\pi / 8\right)}{\sqrt{2 \pi}\left[\cos ^{2}\left(\varphi_{0} / 2-\pi / 8\right)-\cos ^{2}\left(\varphi_{0} / 2+3 \pi / 8\right)\right]} .
$$

This coefficient is continuous at $\varphi_{0}=\frac{1}{4} \pi$, it is discontinuous and unbounded as $\varphi_{0}=-\frac{1}{4} \pi$ or $\varphi_{0}=\frac{3}{4} \pi$, and $D_{\infty}=0$ if $\varphi_{0}=-\frac{3}{4} \pi$.

As $|\operatorname{Im} \gamma|$ grows the numerical values of the backscattering coefficient evaluated by the formulae (8.19), (8.20) approach the limiting value $D_{\infty}\left(\varphi_{0}\right)$. Figure 9 shows the real part of $D_{\infty}\left(\varphi_{0}\right)$ $\left(\operatorname{Im} D_{\infty}=\operatorname{Re} D_{\infty}\right)$ and the real and imaginary parts of $D\left(\varphi_{0}\right)$ for $\theta=\pi / 3+i \nu, v=4$ $(\gamma=23.650+13.645 i)$. Apart from small neighbourhoods of the points $\varphi_{0}=-\frac{3}{4} \pi$ and $\varphi_{0}=\frac{1}{4} \pi$, where $D\left(\varphi_{0}\right)$ is discontinuous and unbounded and $D_{\infty}\left(\varphi_{0}\right)$ is continuous, the coefficients $D$ and $D_{\infty}$ are very close to each other. The difference $\left|D-D_{\infty}\right|$ decreases as $|v|$ approaches 6 and $|\nu| \leqslant 6$. For $\theta=\frac{1}{3} \pi+6 i$ the impedance parameter becomes $\gamma=174 \cdot 69+100 \cdot 86 i$. The numerical 


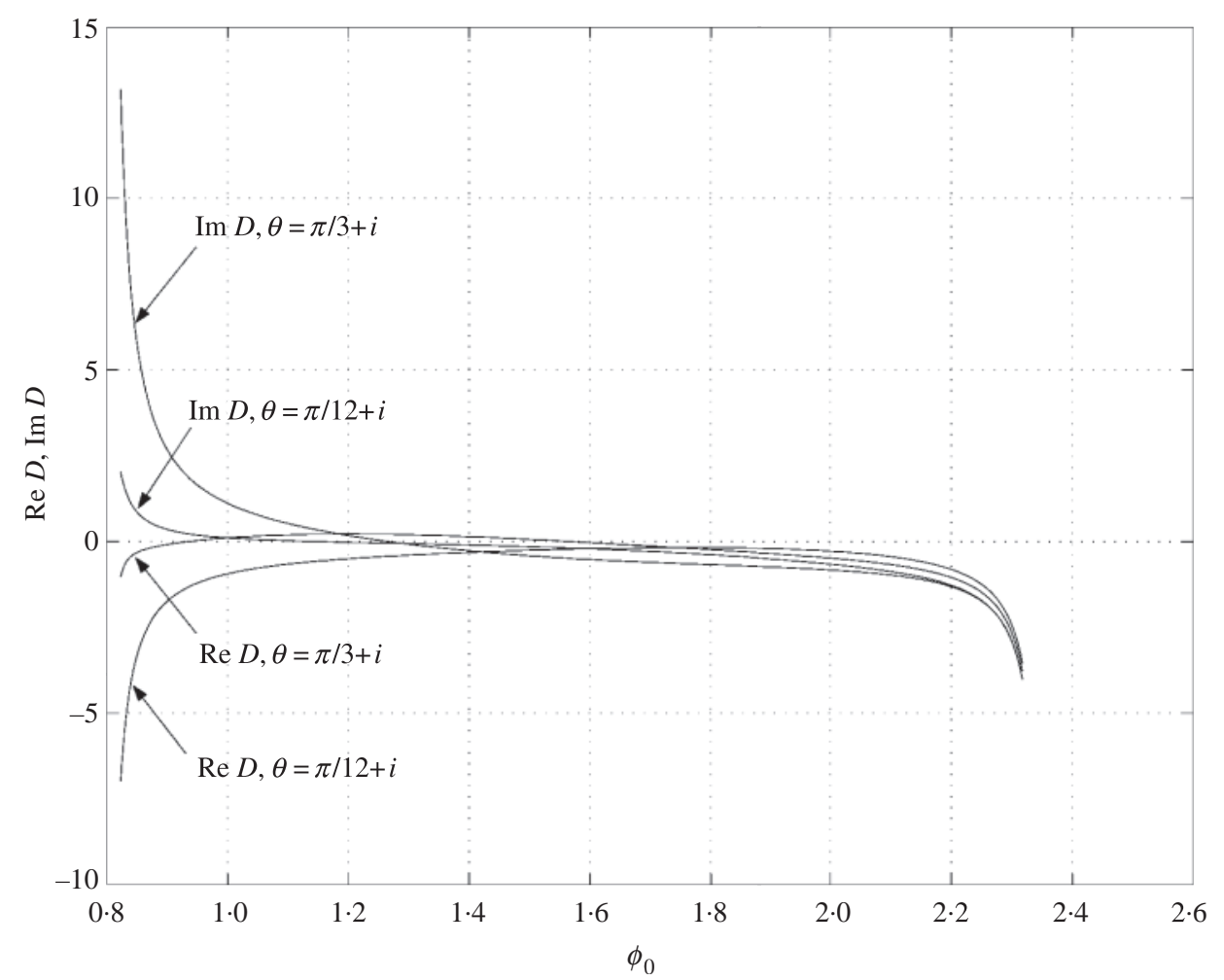

Fig. 8 The real and imaginary parts of the backscattering coefficient for $\varphi_{0} \in(\pi / 4,3 \pi / 4)$

procedure is unstable for $v>6$. This is because the critical points $z_{1}, t_{1},-1 / k$ and 1 are very close to each other. For example, for $v=6 z_{1}=1.000575-0.000332 i, t_{1}=-0.992-0.00459 i$ and $-1 / k=0.9926-0.0043 i$.

Clearly, in the case of no branch points the computer implementation becomes even simpler.

\section{Conclusion}

We have found a closed-form solution of the problem on electric polarization of a right-angled magnetically conductive wedge. The problem has been reduced to the second-order difference equation (3.7) with the shift $\pi$ and $2 \pi$-periodic coefficients. To solve this equation we have analysed the auxiliary equation (3.10) whose shift is $2 \pi$ and coefficients are $\pi$-periodic functions. On using the new method for difference equations based on the theory of the Riemann-Hilbert problem on Riemann surfaces introduced in (6), we have solved the auxiliary equation in terms of two quadratures (4.63). The corresponding Riemann surface is a torus, and because of the choice of the branch cuts, we have managed to solve the Jacobi inversion problem without elliptic functions.

We have also proposed a new procedure for the general solution of a second-order difference 


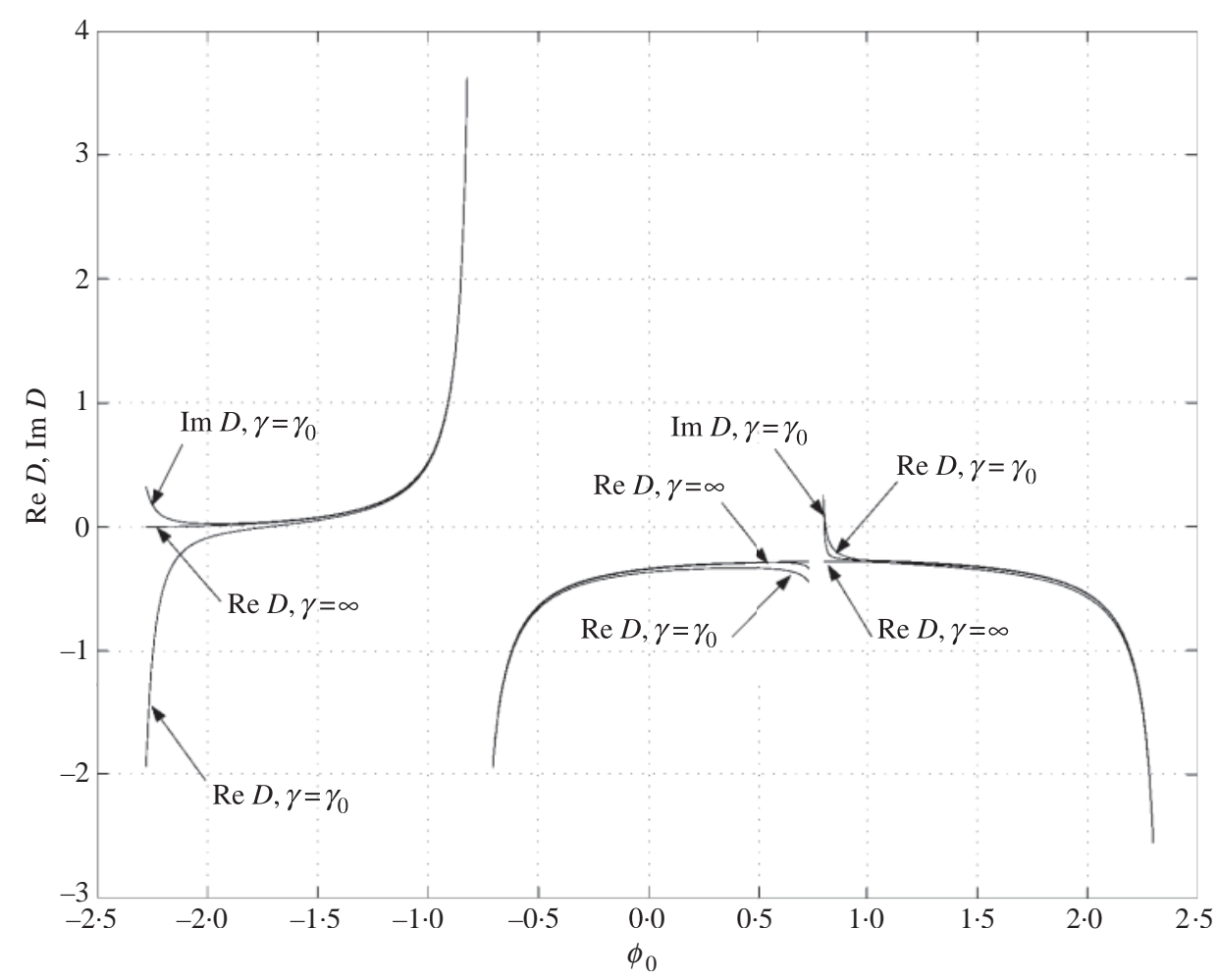

Fig. 9 The real and imaginary parts of the backscattering coefficient for the impedance parameters $\gamma=\gamma_{0}=27 \cdot 31+i 15 \cdot 76(\theta=\pi / 3+4 i)$ and $\gamma=\infty$

equation when the period of the coefficients is twice as much as the shift. It indicates which solutions of the auxiliary equation form the basis of the solutions and which ones are mock solutions.

The spectral functions $\mathcal{S}_{1}(s)$ and $\mathcal{S}_{2}(s)$ found in the paper are meromorphic single-valued functions in the complex plane. In the strip $-3 \pi / 4 \leqslant \operatorname{Re} s \leqslant 3 \pi / 4$ the function $\mathcal{S}_{1}(s)$ has a prescribed pole and it decays exponentially: $\mathcal{S}_{1}(s)=O\left(e^{-\frac{1}{3}|\operatorname{Im} s|}\right), \operatorname{Im} s \rightarrow \pm \infty$. As for the second function, it is free of poles in the strip $-\pi / 4 \leqslant \operatorname{Re} s \leqslant \pi / 4$ and bounded as $\operatorname{Im} s \rightarrow \pm \infty$. It has definite finite limits at infinity, and $\mathcal{S}_{2}(i \infty)=-\mathcal{S}_{2}(-i \infty)$.

The procedure presented is viable and sufficient for numerical purposes. To compute the diffraction coefficient, for example, it is required to evaluate three integrals. For the backscattering coefficient, one needs just two integrals from (8.17), $\mathcal{J}_{1}(z), \mathcal{J}_{2}(z)$, and their first derivatives $\mathcal{J}_{1}^{\prime}(z)$, $\mathcal{J}_{2}^{\prime}(z)$. As the impedance parameter $\gamma \rightarrow \infty$ the values of the backscattering coefficient $D\left(\varphi_{0}\right)$ approach the value $D_{\infty}\left(\varphi_{0}\right)$ of the coefficient when the magnetically conductive screen vanishes.

The method presented in (6) and this paper can be applied to governing second-order difference equations of the diffraction theory with any finite number of zeros of the discriminant of the equation. 


\section{Acknowledgements}

The work was partly supported by Louisiana Board of Regents grant LEQSF(2002-04)-ENH-TR-13 and the Russian Foundation for Basic Research grant 04-01-00160. The authors are grateful to the referees for writing meaningful and thorough reviews.

\section{References}

1. T. B. A. Senior and J. L. Volakis, Approximate Boundary Conditions in Electromagnetics (The Institution of Electrical Engineers, London 1995).

2. G. D. Maliuzhinets, Excitation, reflection and emission of surface waves from a wedge with given face impedances, Sov. Phys. Dokl. 3 (1958) 752-755.

3. C. Demetrescu, C. C. Constantinou and M. J. Mehler, Diffraction by a right-angled resistive wedge, Radio Science 33 (1998) 39-53.

4. —,,-- and B. V. Budaev, Diffraction by a resistive sheet attached to a two-sided impedance plane, Electromagnetics 18 (1998) 315-332.

5. T. B. A. Senior and S. R. Legault, Second-order difference equations in diffraction theory, Radio Science 35 (2000) 683-690.

6. Y. A. Antipov and V. V. Silvestrov, Second-order functional-difference equations. I: method of the Riemann-Hilbert problem on Riemann surfaces, Q. Jl Mech. Appl. Math. 57 (2004) 245-265.

7. S. R. Legault and T. B. A. Senior, Solution of a second-order difference equation using the bilinear relations of Riemann, J. Math. Phys. 43 (2002) 1598-1621.

8. Y. A. Antipov and V. V. Silvestrov, Vector functional-difference equation in electromagnetic scattering, IMA J. Appl. Math. 69 (2004) 27-69.

9. F. D. Gakhov, Boundary Value Problems (Pergamon Press, Oxford 1966).

\section{APPENDIX A}

Analysis of the method by Demetrescu et al.

We aim to show that the procedure $(3,4)$ does not give the general solution of the problem, and the number of arbitrary constants is less than it is required by the physical problem. For illustration, we choose the simplest case $\eta=0\left(\sin \theta=\frac{1}{2} \sqrt{3}\right)$ when there are no branch points. According to $(3,4)$ the general odd solution of equation (3.7) has the form

$$
f(s)=f_{*}(s)-f_{*}(-s)
$$

where

$$
f_{*}(s)=\hat{f}(s) M(s)+\left(1-\frac{\sin \theta}{\cos s}\right)[\hat{f}(s+\pi)+\hat{f}(s-\pi)] M(s+\pi), \quad-\pi \leqslant \operatorname{Re} s \leqslant \pi,
$$

and $M(s)$ is a $2 \pi$-periodic meromorphic function to be determined. As for the function $\hat{f}(s)$, it is a partial solution to the auxiliary equation (3.10) given by

$$
\begin{gathered}
\hat{f}(s)=-\frac{b(s) \varphi_{+}(s)+\sqrt{3} \sin s \cos s \varphi_{-}(s)}{2 a(s)}, \quad-3 \pi \leqslant \operatorname{Re} s \leqslant-\pi, \\
\hat{f}(s)=\varphi_{+}(s), \quad-\pi \leqslant \operatorname{Re} s \leqslant \pi, \\
\hat{f}(s)=\frac{-b(s) \varphi_{+}(s)+\sqrt{3} \sin s \cos s \varphi_{-}(s)}{2 a(s)}, \quad \pi \leqslant \operatorname{Re} s \leqslant 3 \pi,
\end{gathered}
$$


where

$$
\varphi_{ \pm}(s)=F_{10}(z) \pm F_{20}(z) .
$$

Here the functions $F_{j 0}(z)(j=1,2)$ are the integrals (7.8). The auxiliary function $f_{*}(s)$ becomes

$$
\begin{gathered}
f_{*}(s)=\varphi_{+}(s) M(s)+\frac{N(s) M(s+\pi)}{2 \cos s+\sqrt{3}}, \quad-\pi \leqslant \operatorname{Re} s \leqslant \pi, \\
N(s)=\left\{\begin{array}{l}
\cos s \varphi_{+}(s+\pi)+\sqrt{3} \sin s \varphi_{-}(s+\pi), \quad 0 \leqslant \operatorname{Re} s \leqslant \pi, \\
\cos s \varphi_{-}(s+\pi)-\sqrt{3} \sin s \varphi_{-}(s+\pi), \quad-\pi \leqslant \operatorname{Re} s \leqslant 0 .
\end{array}\right.
\end{gathered}
$$

It can be verified that the function $f_{*}(s)$ is continuous through the imaginary axis and is analytic in the strip $-\pi \leqslant \operatorname{Re} s \leqslant \pi$. Next, by the technique presented in section 7 we find

$$
\begin{array}{cc}
F_{10}\left(\frac{1}{z}\right)=C_{*} r_{+}(z) F_{10}(z), & F_{20}\left(\frac{1}{z}\right)=\frac{F_{20}(z)}{C_{*} r_{+}(z)}, \quad 0 \leqslant \operatorname{Re} s \leqslant \pi, \\
F_{10}\left(\frac{1}{z}\right)=C_{*} r_{-}(z) F_{10}(z), & F_{20}\left(\frac{1}{z}\right)=\frac{F_{20}(z)}{C_{*} r_{-}(z)}, \quad-\pi \leqslant \operatorname{Re} s \leqslant 0,
\end{array}
$$

where

$$
r_{ \pm}(z)=\frac{z \pm(2-\sqrt{3}) i}{z \pm(2+\sqrt{3}) i}, \quad C_{*}=2+\sqrt{3} .
$$

Let first $0 \leqslant \operatorname{Re} s \leqslant \pi$. On using (A.4) and (A.6) and the relations

$$
\begin{gathered}
\varphi_{ \pm}(s+\pi)=F_{1}(1 / z) \pm F_{2}(1 / z), \\
C_{*} r_{+}(z)+\frac{1}{C_{*} r_{+}(z)}=\frac{4\left(z^{2}+i z-1\right)}{z^{2}+4 i z-1}, \quad C_{*} r_{+}(z)-\frac{1}{C_{*} r_{+}(z)}=\frac{2 \sqrt{3}\left(z^{2}+1\right)}{z^{2}+4 i z-1}
\end{gathered}
$$

we derive

$$
N(s)=2 \cos s \varphi_{+}(s)+\sqrt{3} \varphi_{-}(s), \quad 0 \leqslant \operatorname{Re} s \leqslant \pi .
$$

It turns out that the above formula is also valid for the strip $-\pi \leqslant \operatorname{Re} s \leqslant 0$. Hence

$$
f_{*}(s)=\varphi_{+}(s) M(s)+\frac{2 \cos s \varphi_{+}(s)+\sqrt{3} \varphi_{-}(s)}{2 \cos s+\sqrt{3}} M(s+\pi), \quad-\pi \leqslant \operatorname{Re} s \leqslant \pi .
$$

Because of the asymptotics of the solution at infinity, the most general form of the function $M(s)$ is given by

$$
M(s)=A_{0}+A_{1} \cos s+B_{1} \sin s .
$$

On using the property of the functions $\varphi_{ \pm}(s)$ to be even and from formula (A.1), the general solution of equation (3.7) becomes

$$
f(s)=2 B_{1} \sin s\left(\varphi_{+}(s)-\frac{2 \cos s \varphi_{+}(s)+\sqrt{3} \varphi_{-}(s)}{2 \cos s+\sqrt{3}}\right) .
$$

So, the two constants $A_{0}$ and $A_{1}$ are eliminated from the representation of the solution. This means that the solution of the problem has only three arbitrary constants $C_{0}, C_{1}$ from (3.13) and $B_{1}$ in (A.12). At the same time, there are five additional conditions to be satisfied: four conditions (6.11) and (6.12) as well as the condition that removes the extra pole in (A.12) at the point $s=\frac{5}{6} \pi$. Thus, the representation (A.1), (A.2) is a partial solution of equation (3.7), not the general one. Note that without the procedure of section 7 , a priori, it is not clear how many constants are missed. 


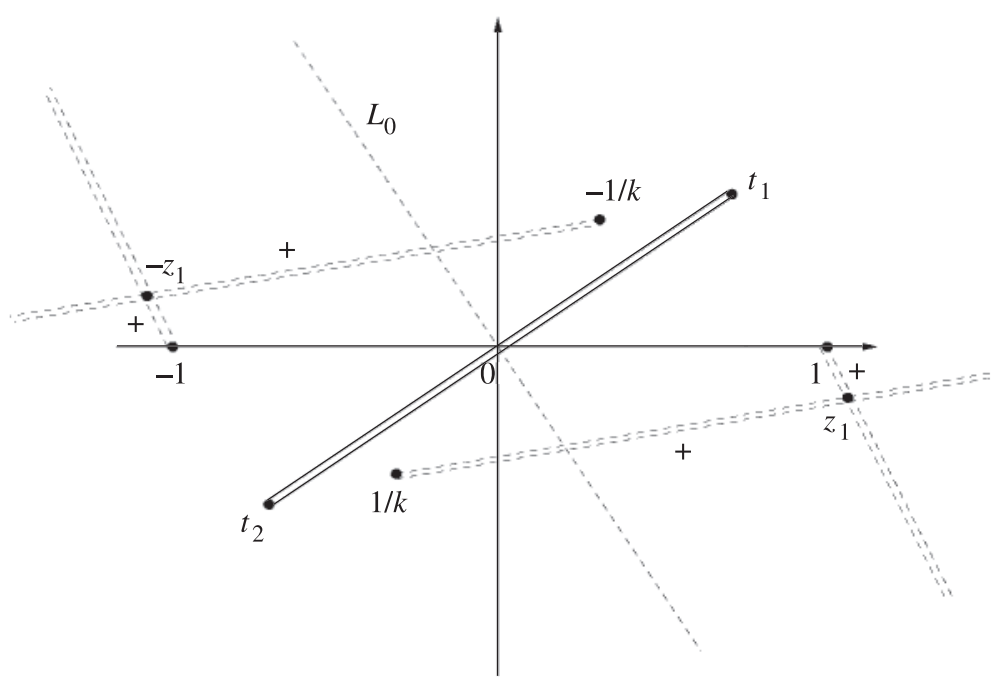

Fig. 10 The branch cuts for the function $q^{1 / 2}(z)$

\section{APPENDIX B}

Evaluation of the function $f^{\prime}(s)$

To evaluate the backscattering coefficient by using formulae (8.19) and (8.20) one needs the derivative $f^{\prime}(s)=$ $B_{0} \mathcal{F}_{0}^{\prime}(s)+B_{1} \mathcal{F}_{1}^{\prime}(s)$, where

$$
\mathcal{F}_{j}^{\prime}(s)=\left[z^{\prime} \chi_{3}^{\prime}(z) \mathcal{H}_{j}(s)+\chi_{3}(z) \mathcal{H}_{j}^{\prime}(s)\right] \cos ^{j} s-\sin s \chi_{3}(z) \mathcal{H}_{j}(s) \delta_{j 1},
$$

$\delta_{j 1}$ is the Kronecker symbol, $j=0,1$,

$$
\begin{gathered}
z^{\prime}=-\frac{1}{2} \tan \frac{\eta}{2} \operatorname{cosec}^{2} \frac{s}{2} \\
\chi_{3}^{\prime}(z)=\frac{z}{\left(z^{2}-1\right) \chi_{3}(z)}\left[2 z^{2}-z_{1}^{2}-t_{1}^{2}-\frac{\left(z^{2}-t_{1}^{2}\right)\left(z^{2}-z_{1}^{2}\right)}{z^{2}-1}\right] \\
\mathcal{H}_{j}(s)=(1-\cos s) \cosh \chi_{4}(z)-\frac{(-1)^{j} i \sin s \beta(s)}{\cos s+\cos \eta} \sinh \chi_{4}(z) \\
\times\left[\left(\cos s \beta(s)+\sin s \beta^{\prime}(s)+\frac{\sin ^{2} s \beta(s)}{\cos s+\cos \eta}\right) \sinh \chi_{4}(z)+\sin s \beta(s) \cosh \chi_{4}(z) \frac{d}{d s} \chi_{4}(z)\right] \\
\mathcal{H}_{j}^{\prime}(s)=\sin s \cosh \chi_{4}(z)+(1-\cos s) \sinh \chi_{4}(z) \frac{d}{d s} \chi_{4}(z)-\frac{(-1)^{j}}{\cos s+\cos \eta} \\
\chi_{4}(z)=q^{1 / 2}(z) \chi_{2}(z), \quad \frac{d}{d s} \chi_{4}(z)=-\frac{z z^{\prime} \chi_{2}(z)}{q^{1 / 2}(z)}\left(1+k^{2}-2 k^{2} z^{2}\right)+z^{\prime} q^{1 / 2}(z) \chi_{2}^{\prime}(z),
\end{gathered}
$$




$$
\begin{gathered}
\beta(s)=-\frac{1}{2} \sin \eta(1-\cos s) q^{1 / 2}(z), \quad \chi_{2}^{\prime}(z)=\mathcal{J}_{1}^{\prime}(z)+\mathcal{J}_{2}^{\prime}(z), \\
\beta^{\prime}(s)=-\frac{1}{2} \sin \eta\left[\sin s q^{1 / 2}(z)-(1-\cos s) \frac{z z^{\prime}\left(1+k^{2}-2 k^{2} z^{2}\right)}{q^{1 / 2}(z)}\right], \\
\mathcal{J}_{1}^{\prime}(z)=-\frac{t_{1}}{\pi i} \int_{0}^{1} \frac{\left[\log l_{1}\left(t_{1} x\right)+\pi i\right]\left(t_{1}^{2} x^{2}+z^{2}\right) d x}{q^{1 / 2}\left(t_{1} x\right)\left(t_{1}^{2} x^{2}-z^{2}\right)^{2}}, \\
\mathcal{J}_{2}^{\prime}(z)=\left(1-z_{1}\right) \int_{0}^{1} \frac{\left(t^{2}+z^{2}\right) d x}{\left(t^{2}-z^{2}\right)^{2} q^{1 / 2}\left(t^{+}\right)}, \quad t=\left(z_{1}-1\right) x+1 .
\end{gathered}
$$

The branch of the logarithmic function has to be fixed by the condition $\log l_{1}\left(t_{1}\right)=-\frac{4}{3} \pi i$. For numerical purposes, we present the chosen branch of the function $q^{1 / 2}(z)$ as follows (Fig.10):

$$
q^{1 / 2}(z)=k\left|q^{1 / 2}(z)\right| e^{i\left(\tau_{1}^{+}+\tau_{1}^{-}+\tau_{2}^{+}+\tau_{2}^{-}\right) / 2},
$$

where

$$
\begin{array}{cl}
\tau_{1}^{ \pm}=\arg (z \pm 1), & \tau_{2}^{ \pm}=\arg \left(z \pm \frac{1}{k}\right), \\
\tau_{1}^{-} \in\left[\alpha_{1}, 2 \pi+\alpha_{1}\right], & \tau_{1}^{+} \in\left[-\pi+\alpha_{1}, \pi+\alpha_{1}\right], \\
\tau_{2}^{-} \in\left[-2 \pi+\alpha_{2}, \alpha_{2}\right], & \tau_{2}^{+} \in\left[-\pi+\alpha_{2}, \pi+\alpha_{2}\right], \\
\alpha_{1}=\tan ^{-1} \frac{\operatorname{Im}\left(z_{1}-1\right)}{\operatorname{Re}\left(z_{1}-1\right)}, & \alpha_{2}=\tan ^{-1} \frac{\operatorname{Im}\left(z_{1}-1 / k\right)}{\operatorname{Re}\left(z_{1}-1 / k\right)} .
\end{array}
$$

For the integrals $\mathcal{J}_{2}(z), \mathcal{J}_{2}^{\prime}(z)$ we need $q^{1 / 2}\left(t^{+}\right)$, where $t^{+}$is a point of the left bank of the cut along the straight line joining the points $z=1$ and $z=z_{1}$. The quantities $\tau_{1}^{ \pm}$and $\tau_{2}^{ \pm}$become

$$
\begin{gathered}
\tau_{1}^{-}=\alpha_{1}, \quad \tau_{1}^{+}=\tan ^{-1} \frac{\operatorname{Im}(z+1)}{\operatorname{Re}(z+1)}, \\
\tau_{2}^{-}=-2 \pi+\tan ^{-1} \frac{\operatorname{Im}(z-1 / k)}{\operatorname{Re}(z-1 / k)}, \quad \tau_{2}^{+}=\tan ^{-1} \frac{\operatorname{Im}(z+1 / k)}{\operatorname{Re}(z+1 / k)} .
\end{gathered}
$$

Finally, we describe how to evaluate the function $\chi_{3}(z)$. For the backscattering coefficient, we have to calculate the function $\chi_{3}(z)$ on the straight line $L_{0}=\left\{z=i t_{1} \tau, \tau \in(-\infty, \infty)\right\}$. The single branch of this function is fixed in section 4.4 and it is discontinuous at the point $z=0$. This function can be written in the form

$$
\chi_{3}(z)=\left|\chi_{3}(z)\right| e^{i\left(\theta_{+}+\theta_{-}+\theta_{2}^{-}-\theta_{1}^{-}+\theta_{2}^{+}-\theta_{1}^{+}\right) / 2},
$$

where

$$
\begin{gathered}
\theta_{ \pm}=\arg \left(z \pm t_{1}\right), \quad \theta_{1}^{ \pm}=\arg (z \pm 1), \quad \theta_{2}^{ \pm}=\arg \left(z \pm z_{1}\right), \\
\alpha \leqslant \theta_{ \pm} \leqslant 2 \pi+\alpha, \quad \alpha=\arg t_{1} \quad\left(\text { for } \operatorname{Im} \theta>0, \quad 0<\arg t_{1}<\pi / 2\right), \\
\alpha_{1} \leqslant \theta_{j}^{-} \leqslant 2 \pi+\alpha_{1}, \quad-\pi+\alpha_{1} \leqslant \theta_{j}^{+} \leqslant \pi+\alpha_{1}, \quad j=1,2 .
\end{gathered}
$$

